\title{
. \\ Search for charged-lepton-flavour violation in Z-boson decays with the ATLAS detector
}

\author{
ATLAS Collaboration ${ }^{\star}$
}

\begin{abstract}
Leptons with essentially the same properties apart from their mass are grouped into three families (or flavours). The number of leptons of each flavour is conserved in interactions, but this is not imposed by fundamental principles. Since the formulation of the standard model of particle physics, the observation of flavour oscillations among neutrinos has shown that lepton flavour is not conserved in neutrino weak interactions. So far, there has been no experimental evidence that this also occurs in interactions between charged leptons. Such an observation would be a sign of undiscovered particles or a yet unknown type of interaction. Here the ATLAS experiment at the Large Hadron Collider at CERN reports a constraint on lepton-flavour-violating effects in weak interactions, searching for Z-boson decays into a $\tau$ lepton and another lepton of different flavour with opposite electric charge. The branching fractions for these decays are measured to be less than $8.1 \times 10^{-6}(e \tau)$ and $9.5 \times 10^{-6}(\mu \tau)$ at the $95 \%$ confidence level using $139 \mathrm{fb}^{-1}$ of proton-proton collision data at a centre-of-mass energy of $\sqrt{\mathrm{s}}=13 \mathrm{TeV}$ and $20.3 \mathrm{fb}{ }^{-1}$ at $\sqrt{s}=8 \mathrm{TeV}$. These results supersede the limits from the Large Electron-Positron Collider experiments conducted more than two decades ago.
\end{abstract}

\begin{abstract}
$\mathrm{n}$ the standard model of particle physics $(\mathrm{SM})^{1-4}$, three lepton families (flavours) exist. The number of leptons of each family is conserved in weak interactions, and violation of this assumption is known as lepton flavour violation (LFV). No fundamental principles forbid LFV processes in the SM. The phenomenon of neutrino oscillations, where neutrinos (the neutral leptons) of one flavour transform into those of another ${ }^{5,6}$, indicates that neutrinos have mass and LFV processes do occur in nature. The mechanisms responsible for neutrinos acquiring mass and weak interactions violating lepton flavour conservation remain unknown. More experimental data are needed to constrain and guide possible generalizations of the SM explaining these phenomena.
\end{abstract}

An observation of LFV in charged-lepton interactions would be an unambiguous sign of new physics. In particular, decays of the $Z$ boson into a light lepton (electron or muon) and a $\tau$ lepton at colliders are of experimental interest. The abundance of $Z$ bosons produced at the Large Hadron Collider (LHC) offers the opportunity to strongly constrain potential LFV $Z \rightarrow e \tau$ or $Z \rightarrow \mu \tau$ interactions, in particular those proportional to the centre-of-mass energy of the decay ${ }^{7}$. Moreover, $Z \rightarrow e \tau, \mu \tau$ decays are less constrained by low-energy experiments than $Z \rightarrow e \mu$ decays. According to current knowledge, these decays can occur via neutrino mixing but are too rare to be detected. Only 1 in approximately $10^{54} Z$ bosons would decay into a muon and a $\tau$ lepton ${ }^{8}$. An observation of such decays would therefore require new theoretical explanations. For example, theories predicting the existence of heavy neutrinos ${ }^{9}$ provide a fundamental understanding of the observed tiny masses and large mixing of SM neutrinos. In such theories, up to 1 in $10^{5} \mathrm{Z}$ bosons would be expected to undergo an LFV decay involving $\tau$ leptons. The ATLAS experiment can test the predictions of such theories by observing or setting ever more stringent constraints on LFV Z-boson decays.

Constraints on the branching fractions $(\mathcal{B})$ of the LFV decays of the $Z$ boson involving a $\tau$ lepton have been set by the experiments at the Large Electron-Positron Collider (LEP): $\mathcal{B}(Z \rightarrow e \tau)<9.8 \times 10^{-6}$ (ref. ${ }^{10}$ ) and $\mathcal{B}(Z \rightarrow \mu \tau)<1.2 \times 10^{-5}$ (ref. ${ }^{11}$ ) at the $95 \%$ confidence level (CL). The ATLAS experiment ${ }^{12}$ at the LHC has set constraints $\mathcal{B}(Z \rightarrow e \tau)<5.8 \times 10^{-5}$ at $95 \% \mathrm{CL}$ using part of the Run 2 data and $\mathcal{B}(Z \rightarrow \mu \tau)<1.3 \times 10^{-5}$ using the Run 1 data and a subset of the Run 2 data $^{13}$.

This work uses proton-proton $(p p)$ collision data collected by the ATLAS experiment during Run 2 of the LHC, containing about eight billion $Z$-boson decays. Only events with a $\tau$ lepton that decays hadronically are considered. Neural network (NN) classifiers are used in a novel way for optimal discrimination of signal from background, and to achieve improved sensitivity in the search for LFV effects in the data using a binned maximum-likelihood fit. The result for the $\mu \tau$ channel is combined with a previous LHC Run 1 result to further improve the sensitivity. These results set constraints on LFV $Z$-boson decays involving $\tau$ leptons that supersede the most stringent ones set by the LEP experiments more than two decades ago.

\section{The ATLAS experiment and data sample}

To record and analyse the LHC $p p$ collisions, the ATLAS experiment uses a multipurpose particle detector with a forward-backward symmetric cylindrical geometry and a near $4 \pi$ coverage in solid angle $\mathrm{e}^{12,14,15}$. It consists of an inner tracking detector surrounded by a superconducting solenoid, electromagnetic and hadronic calorimeters, and a muon spectrometer.

The search uses the complete dataset of $p p$ collision events at a centre-of-mass energy of $\sqrt{s}=13 \mathrm{TeV}$ collected by the ATLAS experiment during LHC Run 2. This dataset was recorded using single-electron or single-muon triggers ${ }^{16}$ and corresponds to an integrated luminosity of $139 \mathrm{fb}^{-1}$. For the search in the $\mu \tau$ channel, the results are combined with those of a previous similar search using $p p$ collisions at $\sqrt{s}=8 \mathrm{TeV}$ during LHC Run 1, corresponding to an integrated luminosity of $20.3 \mathrm{fb}^{-1}$ ( ref. ${ }^{17}$ ).

Candidates for electrons ${ }^{18}$, muons ${ }^{19}$, jets ${ }^{20-22}$, and visible decay products of hadronic $\tau$-lepton decays $\left(\tau_{\text {had-vis }}\right)^{23,24}$ are reconstructed from energy deposits in the calorimeters and charged-particle tracks measured in the inner detector and the muon spectrometer. 
Electron candidates are required to pass the Medium likelihood-based identification requirement ${ }^{18}$ and have pseudorapidity $|\eta|<1.37$ or $1.52<|\eta|<2.47$. Muon candidates are required to pass the Medium identification requirement ${ }^{19}$ and have $|\eta|<2.5$. Both the electron and muon candidates must have transverse momentum $p_{\mathrm{T}}>30 \mathrm{GeV}$ and satisfy the Tight isolation requirement ${ }^{18,19}$. The lower bounds on the electron and muon transverse momenta are driven by the acceptance of the trigger selection.

Quark- or gluon-initiated particle showers (jets) are reconstructed using the anti- $k_{t}$ algorithm ${ }^{20,21}$ with the radius parameter $R=0.4$. Jets fulfilling $p_{\mathrm{T}}>20 \mathrm{GeV}$ and $|\eta|<2.5$ are identified as containing $b$ hadrons if tagged by a dedicated multivariate algorithm ${ }^{25}$.

The $\tau_{\text {had-vis }}$ candidates are reconstructed from jets with $p_{\mathrm{T}}>10 \mathrm{GeV}$, $|\eta|<1.37$ or $1.52<|\eta|<2.5$, and one or three associated tracks, referred to as '1-prong' (1P) and '3-prong' (3P), respectively. The $\tau_{\text {had-vis }}$ identification is performed by a recurrent $\mathrm{NN}$ algorithm ${ }^{23}$, which uses calorimetric shower shapes and tracking information to discriminate true $\tau_{\text {had-vis }}$ candidates from fake candidates from quarkor gluon-initiated jets. The $\tau_{\text {had-vis }}$ candidates are required to pass the Tight identification selection, which has an efficiency of $60 \%$ (45\%) for true $1 \mathrm{P}(3 \mathrm{P}) \tau_{\text {had-vis }}$ candidates, constant in the $\tau_{\text {had-vis }}$ candidates transverse momentum, and a misidentification rate of 1 in $70(700)$ for fake 1P (3P) candidates in dijet events. Dedicated multivariate algorithms are used to further discriminate between $\tau_{\text {had-vis }}$ and electrons, and to calibrate the $\tau_{\text {had-vis }}$ energy ${ }^{24}$. The $\tau_{\text {had-vis }}$ candidate with the largest $p_{\mathrm{T}}$ in each event is the selected candidate and is required to have $p_{\mathrm{T}}>25 \mathrm{GeV}$. Based on simulation, in $Z \rightarrow \ell \tau$ decays, the $\tau_{\text {had-vis }}$ candidate is expected to be correctly selected $98 \%$ of the time.

The missing transverse momentum $\left(E_{\mathrm{T}}^{\mathrm{miss}}\right)$ is calculated as the negative vectorial sum of the $p_{\mathrm{T}}$ of all fully reconstructed and calibrated physics objects ${ }^{26,27}$. The calculation also includes inner detector tracks that originate from the vertex associated with the hard-scattering process but are not associated with any of the reconstructed objects. The missing transverse momentum is the best proxy for the total transverse momentum of undetected particles (in particular neutrinos) in an event.

\section{Search strategy}

The $Z \rightarrow \ell \tau \rightarrow \ell \tau_{\text {had-vis }}+\nu(\ell=$ light lepton, $e$ or $\mu)$ signal events have a number of key features that can be exploited to separate them from the SM background events. The signal events are characterized by their unique final state, which has exactly one $\ell$ and one $\tau$ lepton, with the invariant mass of the pair being compatible with the $Z$-boson mass. The $\ell$ and $\tau$ leptons carry opposite electric charges and are emitted approximately back to back in the plane transverse to the proton beam direction. Since the $\tau$ lepton is typically boosted due to the large difference between its mass and the mass of its parent $Z$ boson, the neutrino from its decay is usually almost collinear with the visible $\tau$-decay products. The neutrino escapes detection and is reconstructed as part of the $E_{\mathrm{T}}^{\text {miss }}$ of the event. In a signal event, this is the only major source of $E_{\mathrm{T}}^{\text {miss }}$.

The major background contributions for this search are as follows: lepton-flavour-conserving $Z \rightarrow \tau \tau \rightarrow \ell \tau_{\text {had-vis }}+3 \nu$ decays, where one of the $\tau$ leptons decays leptonically and the other hadronically; $Z \rightarrow \ell \ell$ decays, where one of the light leptons is misidentified as the $\tau_{\text {had-vis }}$ candidate; events with a quark- or gluon-initiated jet that is misidentified as the $\tau_{\text {had-vis }}$ candidate. The last of these are hereafter referred to as events with 'fakes' and are mostly $W(\rightarrow \ell \nu)+$ jets events and purely hadronic multijet events. Other SM processes with a real $\ell \tau_{\text {had-vis }}$ final state, such as decays of a top-antitop-quark pair, two gauge bosons or a Higgs boson, and those with a real $\tau_{\text {had-vis }}$ and a jet misidentified as a light lepton, such as $W(\rightarrow \tau \nu)+$ jets, are considered, although their contribution to the overall background is minor.

The signal and background events are separated by using a set of event selection criteria that help to define a signal-enhanced
Table 1 | Main selection criteria for events in the signal region

\begin{tabular}{|c|c|}
\hline Main selection criteria & Purpose \\
\hline At least one $\tau_{\text {had-vis }}$ candidate & \multirow{3}{*}{$\begin{array}{l}\text { Select events with } \ell-\tau \text { pair } \\
\text { candidate }\end{array}$} \\
\hline Exactly one isolated light lepton & \\
\hline Opposite-sign charged $\ell-\tau_{\text {had-vis }}$ pair & \\
\hline$m_{\mathrm{T}}\left(\tau_{\text {had-vis }}, E_{\mathrm{T}}^{\text {miss }}\right)<35 \mathrm{GeV}$ & Reject $Z \rightarrow \tau \tau$ and $W+$ jets events \\
\hline$m_{\text {vis }}\left(\ell, \tau_{\text {had-vis }}\right)>60 \mathrm{GeV}$ & $\begin{array}{l}\text { Invariant mass of the } \ell-\tau_{\text {had-vis }} \text { pair. } \\
\text { Reject events incompatible with } \\
\ell-\tau \text { pairs from } Z \text {-boson decays }\end{array}$ \\
\hline No tagged $b$-hadron jets & $\begin{array}{l}\text { Reject } t \bar{t} \text { and single-top-quark } \\
\text { events }\end{array}$ \\
\hline $\begin{array}{l}\text { Combined NN output }>0.1(0.2) \text { for } \\
\text { events with } 1 \mathrm{P}(3 \mathrm{P}) \tau_{\text {had-vis }} \text { candidates }\end{array}$ & Reject background-like events \\
\hline $\begin{array}{l}\text { NN (optimized for signal versus } \\
Z \rightarrow \ell \ell \text { ) output }>0.2\end{array}$ & $\begin{array}{l}\text { Ensure orthogonal region for } \\
\text { correcting } Z \rightarrow \ell \ell \text { simulation } \\
\text { ( } \ell \text { misidentified as } 1 \mathrm{P} \tau_{\text {had-vis }} \\
\text { candidate, see section 'Signal and } \\
\text { background predictions') }\end{array}$ \\
\hline
\end{tabular}

sample, referred to as the signal region (SR). The main selection criteria are listed in Table 1 and will be explained in the following. They are primarily based on the multiplicity of reconstructed particle candidates and the event topology, in particular the transverse masses $\left(m_{\mathrm{T}}\right)$, which are defined as

$$
m_{\mathrm{T}}\left(X, E_{\mathrm{T}}^{\mathrm{miss}}\right) \equiv \sqrt{2 p_{\mathrm{T}}(X) E_{\mathrm{T}}^{\mathrm{miss}}\left(1-\cos \left(\phi_{X}-\phi_{E_{\mathrm{T}}^{\mathrm{miss}}}\right)\right)}
$$

where $X$ is either a light lepton or a $\tau_{\text {had-vis }}$ candidate and $\phi$ denotes the azimuthal angle. A schematic of the expected signal and background topologies is described in Extended Data Figs. 1 and 2.

$\mathrm{NN}$ binary classifiers are used to distinguish signal events from $W+$ jets, $Z \rightarrow \tau \tau$ and $Z \rightarrow \ell \ell$ background events. The NNs are trained on simulated events ('Signal and background predictions'). Each individual NN is optimized to discriminate against a particular background process in a given decay channel. The input to these NNs is a mixture of low-level and high-level kinematic variables, as detailed in Methods. The low-level variables are the momentum components of the reconstructed $\ell, \tau_{\text {had-vis }}$ candidate and $E_{\mathrm{T}}^{\mathrm{miss}}$. The high-level variables are kinematic properties of the $\ell-\tau_{\text {had-vis }}-E_{\mathrm{T}}^{\text {miss }}$ system, such as the collinear mass $m_{\text {coll }}(\ell, \tau)$, defined as the invariant mass of the $\ell-\tau_{\text {had-vis }}-\nu$ system, where the $\nu$ is assumed to have a momentum that is equal in $p_{\mathrm{T}}$ and $\phi$ to the measured $E_{\mathrm{T}}^{\mathrm{miss}}$ and equal in $\eta$ to the $\tau_{\text {had-vis }}$ momentum. Given the finite training-sample size, the high-level variables help the $\mathrm{NNs}$ to converge faster, while the NNs exploit any residual correlations between the low-level variables.

The outputs from the individual NNs are numbers between 0 and 1 that reflect the probability for an event to be a signal event; they are combined into a final discriminant, hereafter referred to as the 'combined NN output'. The combination is parameterized by weights associated with each individual NN and optimized for discrimination among various background processes distributed differently along the range of combined NN output values, as detailed in Methods. This allows the maximum-likelihood fit to determine the background contributions more precisely, which ultimately improves the sensitivity.

Events classified by the NNs as being background-like are excluded from the SR, as indicated in Table 1. The signal acceptance times selection efficiency in the SR is $2.7 \%$ for the e $\tau$ channel and $3.0 \%$ for the $\mu \tau$ channel, as determined from simulated signal samples. 

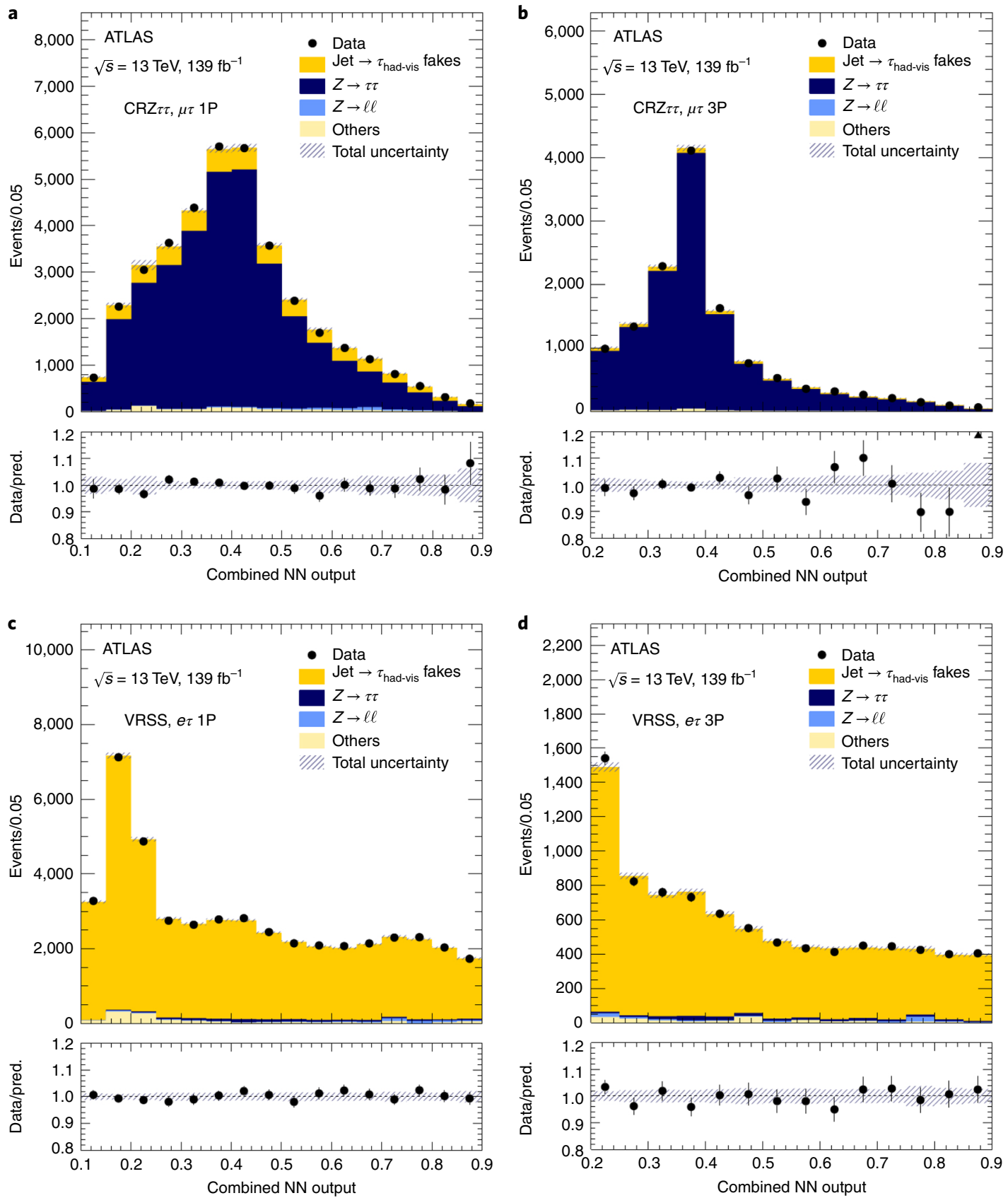

Fig. 1 | Distributions of the combined NN output in control regions and validation regions. a,b, CRZ $\tau \tau$ for the $\mu \tau$ channel with $1 \mathrm{P}(\mathbf{a})$ and $3 \mathrm{P}(\mathbf{b}) \tau_{\text {had-vis }}$ candidates. c,d, Same-sign validation region (VRSS) for the e $\tau$ channel for events with $1 \mathrm{P}(\mathbf{c})$ and $3 \mathrm{P}(\mathbf{d}) \tau_{\text {had-vis }}$ candidates. The expected contributions are determined in a fit to data ('Constraints on $\mathcal{B}(Z \rightarrow \ell \tau)$ )'. The panels below each plot show the ratios of the observed yields to the best-fit background yields. The hatched error bands represent a one standard deviation of the combined statistical and systematic uncertainties. The statistical uncertainties on the data are shown as vertical bars. The last bin in each plot includes overflow events. Similarly good agreement is observed in the same-sign validation region for the $\mu \tau$ channel and $C R Z \tau \tau$ for the $e \tau$ channel, which are not shown here.

\section{Signal and background predictions}

Predictions for signal and background contributions to the event yield and kinematic distributions in the SR are based partly on Monte Carlo (MC) simulations and partly on the use of data in regions that are enriched in background events and do not overlap with the SR.

The signal events were simulated using PYTHIA $8^{28}$ with matrix elements calculated at leading order (LO) in the strong coupling constant $\left(\alpha_{\mathrm{s}}\right)$. Parameter values for initial-state radiation, multiparton interactions and beam remnants were set according to the A14 set of tuned parameters (tune) ${ }^{29}$ with the NNPDF 2.3 LO parton distribution function (PDF) set ${ }^{30}$. Nominal signal samples were generated with a parity-conserving $Z \ell \tau$ vertex and unpolarized $\tau$ leptons. Scenarios where the decays are maximally parity-violating were considered by reweighting the simulated events using TAUSPINNER $^{31}$. The event weight was computed as the probability of occurrence of each generated signal event, based on its kinematics, when assuming a specific $\tau$-polarization state (left-handed or right-handed).

Background $Z \rightarrow \tau \tau$ events were simulated with the SHERPA 2.2.1 ${ }^{32}$ generator using the NNPDF 3.0 NNLO PDF set ${ }^{33}$ and nextto-leading-order (NLO) matrix elements for up to two partons, 
Table 2 I Summary of the uncertainties and their impacts on the measured branching fraction $\mathcal{B}(Z \rightarrow \ell \tau)$

Source of uncertainty

Uncertainty on

$\mathcal{B}(Z \rightarrow \ell \tau)\left(\times 10^{-6}\right)$

e $\tau \quad \mu \tau$

Statistical

$\pm 3.5$

$\pm 2.8$

Systematic

$\pm 2.3$

$\pm 1.6$

$\tau$ leptons

$\pm 1.9$

$\pm 1.5$

Energy calibration

$\pm 1.3$

$\pm 1.4$

Jet rejection

$\pm 0.3$

$\pm 0.3$

Electron rejection

$\pm 1.3$

Light leptons

$\pm 0.4$

$\pm 0.1$

$E_{\mathrm{T}}^{\mathrm{miss}}$, jets and flavour tagging

$\pm 0.6$

$\pm 0.5$

Z-boson modelling

$\pm 0.7$

$\pm 0.3$

Luminosity and other minor backgrounds

$\pm 0.8$

Total

$\pm 4.1$

$\pm 3.2$

The statistical uncertainties include those in the determination of the yields of the events with fakes and from $Z \rightarrow \tau \tau$ or $Z \rightarrow \ell \tau$ decays. The uncertainties related to light leptons include those in the trigger, reconstruction, identification and isolation efficiencies, as well as energy calibrations. The uncertainties related to jets and $E_{T}^{\text {miss }}$ include those in energy calibration and resolution. The uncertainties related to the $Z$-boson modelling include those in the correction of the simulated transverse momentum and the measured production cross-section of the $Z$ boson.

and LO matrix elements for up to four partons, calculated with the COMIX $^{34}$ and OPENLOOPS ${ }^{35-37}$ libraries. They were matched with the SHERPA parton shower ${ }^{38}$ using the MEPS@NLO prescription $^{39-42}$ with the default SHERPA tune. This set-up follows the recommendations of the SHERPA authors. Background $Z \rightarrow \ell \ell$ events were simulated using the POWHEG-BOX ${ }^{43}$ generator with NLO matrix elements and interfaced to PYTHIA 8 to model the parton showers, hadronization and underlying events. All MC samples include a detailed simulation of the ATLAS detector with GEANT $4^{44}$, to produce predictions that can be compared with the data. Furthermore, simulated inelastic $p p$ collisions, generated with PYTHIA 8 using the NNPDF 2.3 LO PDF set and the A3 tune ${ }^{45}$ were overlaid on the hard-scattering events to model the additional $p p$ collisions occurring in the same proton bunch crossing. All simulated events were processed using the same reconstruction algorithms as used for data.

The simulation of $Z$-boson production is improved with a correction derived from measurements in data. The simulated $p_{\mathrm{T}}$ spectra of the $Z$ boson are reweighed to match the unfolded distribution measured by ATLAS in ref. ${ }^{46}$. This improves the predictions of signal, $Z \rightarrow \tau \tau$ and $Z \rightarrow \ell \ell$ events, which are simulated at different orders in $\alpha_{\mathrm{s}}$ using different generators. It also reduces the uncertainties related to missing higher orders in $\alpha_{s}$.

The predicted overall yields of signal and $Z \rightarrow \tau \tau$ events are determined by a binned maximum-likelihood fit to data (see next section) in the SR and in a control region enhanced in $Z \rightarrow \tau \tau \rightarrow \ell \tau_{\text {had-vis }}+3 \nu$ events (CRZ $\left.\tau \tau\right)$, using an unconstrained fit parameter, which accounts for theoretical uncertainties in the total $Z$-boson production cross-section $\left(\sigma_{z}\right)$, as well as the experimental uncertainties related to the acceptance of the common $\ell \tau_{\text {had-vis }}$ final state. The selection criteria for events in the CRZ $\tau \tau$ are the same as those for events in the SR, except that events are required to have $m_{\mathrm{T}}\left(\tau_{\text {had-vis }}, E_{\mathrm{T}}^{\text {miss }}\right)>35 \mathrm{GeV}, m_{\mathrm{T}}\left(\ell, E_{\mathrm{T}}^{\text {miss }}\right)<40 \mathrm{GeV}$ and $70 \mathrm{GeV}<m_{\text {coll }}(\ell, \tau)<110 \mathrm{GeV}$.

A much smaller contribution to the total background originates from $Z \rightarrow \ell \ell$ events. Their predicted overall yield is based on the measured value of $\sigma_{Z}$ (ref. ${ }^{47}$ ) times the measured integrated luminosity. The uncertainty in the measurement is taken into account. The predicted rates of misidentifying electrons and muons in
$Z \rightarrow \ell \ell$ events as $1 \mathrm{P} \tau_{\text {had-vis }}$ candidates are corrected using data in a region enriched in $Z \rightarrow \ell \ell$ events and orthogonal to the SR, where the last selection criterion in Table 1 is inverted and the outputs of the NN classifiers optimized to reject $Z \rightarrow \tau \tau$, and $W+$ jets events are required to be greater than 0.8 . The corrections are derived as functions of $p_{\mathrm{T}}$ and $|\eta|$ of the $\tau_{\text {had-vis }}$ candidate. Statistical uncertainties in the correction are considered.

Events with fakes are one of the dominant contributions to the background, and are estimated from data using the 'fake-factor method', which is described in ref. ${ }^{13}$. A fake factor is defined as the ratio of the number of events with a fake $\tau_{\text {had-vis }}$ candidate passing the Tight $\tau_{\text {had-vis }}$ identification requirement to those failing it. Four fake factors, one for each of the most important backgrounds with fakes $(W(\rightarrow \ell \nu)+$ jets, multijet, $Z(\rightarrow \ell \ell)+$ jets and $t \bar{t}$ events $)$, are measured in data in four corresponding fakes-enriched regions. Each of these regions has a dominant contribution from one of the four targeted backgrounds with fakes. These regions do not overlap with any of the regions used in the final maximum-likelihood fit. The purity of the multijet-enriched region is improved by introducing two additional selection criteria: events must have a same-sign charged $\ell-\tau_{\text {had-vis }}$ pair and $m_{\mathrm{T}}\left(\ell, E_{\mathrm{T}}^{\mathrm{miss}}\right)<40 \mathrm{GeV}$. The fake factors are measured as functions of the transverse momentum of the $\tau_{\text {had-vis }}$ candidate, separately for $e \tau$ and $\mu \tau$ events and for events with $1 \mathrm{P}$ or $3 \mathrm{P} \tau_{\text {had-vis }}$ candidates.

The number of events with a fake $1 \mathrm{P}$ or $3 \mathrm{P} \tau_{\text {had-vis }}$ candidate in a given $p_{\mathrm{T}}$ range in the SR or CRZ $\tau \tau$ is estimated by the number of events with a $\tau_{\text {had-vis }}$ candidate failing the Tight identification requirement, but otherwise satisfying all other selection criteria for that region, multiplied by an average of the fake factors. To calculate this average, the fake factors are summed with weights equal to the expected relative contribution of the corresponding background to the total yield of events in the region with the inverted identification requirement. This approach is used to model the kinematic properties of the events with fakes. The total predicted yields of these events in the SR and CRZ $\tau \tau$ are instead determined by a maximum-likelihood fit to data (see next section), separately for events with $1 \mathrm{P}$ and $3 \mathrm{P} \tau_{\text {had-vis }}$ candidates. This approach avoids the uncertainties associated with the simulation of events with fakes, and makes full use of the large amount of data collected.

The remaining background processes (summarized as 'Others' in the following) have relatively small contributions in the SR and are estimated using simulations. They include events from the production and decays of top quarks, pairs of gauge bosons, the Higgs boson and $W(\rightarrow \tau \nu)+$ jets. The yields of these events are normalized to their theoretical cross-sections.

The modelling of the estimated background is validated using events in regions where a possible contamination from signal is negligible. Especially important to the search is the modelling of the combined NN output distribution of $Z \rightarrow \tau \tau$ events and events with fakes. This is validated by comparing the predicted distributions with data in the CRZ $\tau \tau$ and in a region similar to the SR, but with events that have same-sign charged $\ell-\tau_{\text {had-vis }}$ pairs, as shown in Fig. 1.

\section{Constraints on $\mathcal{B}(Z \rightarrow \ell \tau)$}

A statistical analysis of the selected events is performed to assess the presence of LFV signal events. The statistical analysis method is detailed in Methods. A simultaneous binned maximum-likelihood fit to the combined NN output in the SR and $m_{\text {coll }}(\ell, \tau)$ in the CRZ $\tau \tau$ is used to constrain uncertainties in the models and extract evidence of a possible signal. The fit is performed independently for the $e \tau$ and $\mu \tau$ channels. Events with $1 \mathrm{P}$ and $3 \mathrm{P} \tau_{\text {had-vis }}$ candidates are considered separately. Hypothesis tests, in which a log-likelihood ratio is used as the test statistic, are used to assess the compatibility between the background and signal models and the data.

There are four unconstrained parameters in the fits: two of them determine the overall yields of events with fake $1 \mathrm{P} \tau_{\text {had-vis }}$ or $3 \mathrm{P} \tau_{\text {had-vis }}$ 

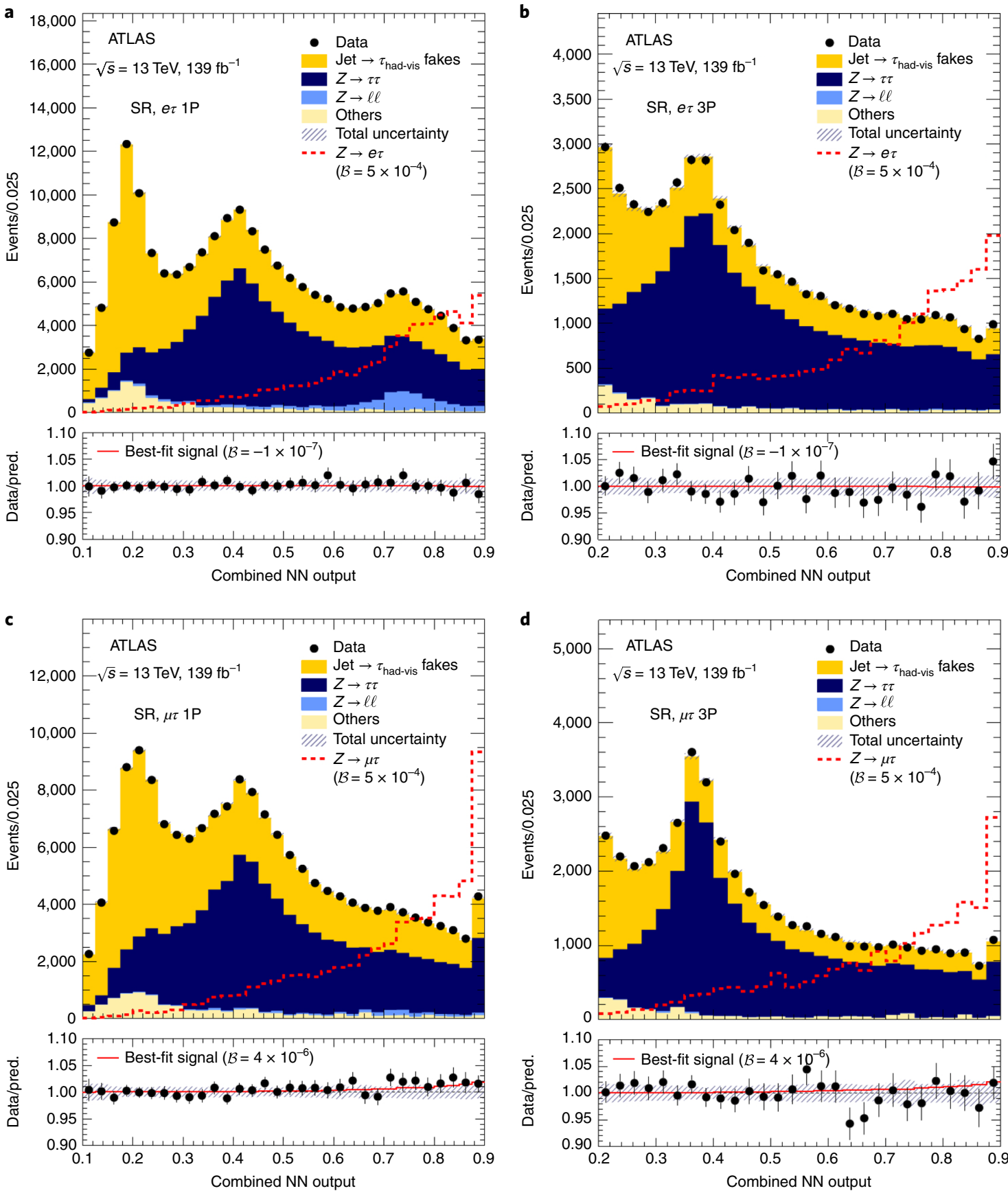

Fig. 2 Distributions of the combined NN output in the signal region. a,b, e $\tau$ events with $1 \mathrm{P}(\mathbf{a})$ and $3 \mathrm{P}(\mathbf{b}) \tau_{\text {had-vis }}$ candidates. $\mathbf{c}, \mathbf{d}, \mu \tau$ events with $1 \mathrm{P}(\mathbf{c})$ and $3 \mathrm{P}(\mathbf{d}) \tau_{\text {had-vis }}$ candidates. The expected contributions are determined in the fit to data. The expected signal, normalized to $\mathcal{B}(Z \rightarrow \ell \tau)=5 \times 10^{-4}$, is shown as a dashed red histogram in each plot. The panels below each plot show the ratios of the observed yields (dots) and the best-fit background-plus-signal yields (solid red line) to the best-fit background yields. The hatched error bands represent a one standard deviation of the combined statistical and systematic uncertainties. The statistical uncertainties on the data are shown as vertical bars. The last bin in each plot includes overflow events.

candidates, one determines $\sigma_{Z}$ times the overall acceptance and reconstruction efficiency of the $\ell \tau_{\text {had-vis }}$ final state in $Z \rightarrow \tau \tau$ and signal events, and the last one, the parameter of interest, determines the LFV branching fraction $\mathcal{B}(Z \rightarrow \ell \tau)$ by modifying an arbitrary pre-fit signal yield.

Constrained parameters are also introduced to account for systematic uncertainties in the signal and background predictions. In the case of no significant deviations from the SM background, exclusion limits are set using the $\mathrm{CL}_{\mathrm{S}}$ method $^{48}$.

Systematic uncertainties in this search include uncertainties in simulated events in the modelling of trigger, reconstruction, identification and isolation efficiencies, as well as energy calibrations and resolutions of reconstructed objects. Conservative theory uncertainties ranging between $4 \%$ and $20 \%$ are also assigned to the predicted cross-sections used for the estimation of minor background processes. These uncertainties are not assigned to events with fakes or Z-boson decays, whose yields are determined from data. These events constitute only a small fraction of the background events in the SR. The dominant uncertainties in this search are those in the overall yields of events with fakes, which are predominantly of statistical nature, and those in the $\tau_{\text {had-vis }}$ energy calibration, which are independent between $1 \mathrm{P}$ and $3 \mathrm{P} \tau_{\text {had-vis }}$ candidates 
Table 3 | Observed number of events and best-fit expected background and signal yields in the SR

\begin{tabular}{|c|c|c|c|c|}
\hline & $\operatorname{SRe} \tau 1 \mathrm{P}$ & SRe $\tau 3 \mathrm{P}$ & $\mathrm{SR} \mu \tau 1 \mathrm{P}$ & $\mathrm{SR} \mu \tau 3 \mathrm{P}$ \\
\hline Observed events & 35,823 & 8,108 & 27,941 & 7,462 \\
\hline Expected SM events & $35,500 \pm 300$ & $8,120 \pm 90$ & $27,100 \pm 200$ & $7,600 \pm 90$ \\
\hline Expected $Z \rightarrow \tau \tau$ events & $17,100 \pm 200$ & $5,420 \pm 70$ & $15,600 \pm 200$ & $5,200 \pm 70$ \\
\hline Expected $Z \rightarrow \ell \ell$ events & $4,200 \pm 200$ & $70 \pm 40$ & $930 \pm 60$ & $12.4 \pm 0.1$ \\
\hline Expected diboson events & $210 \pm 20$ & $66 \pm 9$ & $240 \pm 30$ & $80 \pm 9$ \\
\hline Expected Higgs-boson events & $210 \pm 10$ & $66 \pm 4$ & $210 \pm 10$ & $68 \pm 4$ \\
\hline Pre-fit expected $Z \rightarrow \ell \tau$ events $\left(\mathcal{B}=1 \times 10^{-5}\right)$ & $670 \pm 20$ & $210 \pm 10$ & $720 \pm 20$ & $230 \pm 10$ \\
\hline Best-fit $Z \rightarrow \ell \tau$ events & $0 \pm 300$ & $0 \pm 80$ & $300 \pm 200$ & $90 \pm 70$ \\
\hline
\end{tabular}

The additional requirement of combined NN output $>0.7$ is included to consider the most signal-like events. The events with $1 \mathrm{P}$ and $3 \mathrm{P} \tau_{\text {had }-v i s}$ candidates are fitted simultaneously. The uncertainties include both the statistical and systematic contributions.

\section{Table 4 | Observed and expected (median) upper limits on the signal branching fraction at $95 \% \mathrm{CL}$}

\begin{tabular}{lll}
$\begin{array}{l}\text { Experiment, polarization } \\
\text { assumption }\end{array}$ & \multicolumn{2}{l}{$\begin{array}{l}\text { Observed (expected) upper limit } \\
\text { on } \mathcal{B}(\boldsymbol{Z} \rightarrow \ell \tau)\left(\times 10^{-6}\right)\end{array}$} \\
\hline & $\mathbf{e} \boldsymbol{\mu} \tau$ \\
\hline ATLAS Run 2, unpolarized $\tau$ & $8.1(8.1)$ & $9.9(6.3)$ \\
\hline ATLAS Run 2, left-handed $\tau$ & $8.2(8.6)$ & $9.5(6.7)$ \\
\hline ATLAS Run 2, right-handed $\tau$ & $7.8(7.6)$ & $10(5.8)$ \\
\hline ATLAS Run 1, unpolarized $\tau^{17}$ & & $17(26)$ \\
\hline $\begin{array}{l}\text { ATLAS Run 1+ Run 2 combination, } \\
\text { unpolarized } \tau\end{array}$ & & $9.5(6.1)$ \\
\hline LEP OPAL, unpolarized $\tau^{10}$ & 9.8 & 17 \\
\hline LEP DELPHI, unpolarized $\tau^{11}$ & 22 & 12 \\
\hline
\end{tabular}

Upper limits for different $\tau$-polarization scenarios are reported. The differences between the observed and expected limits are due to the non-zero best-fit signal branching fractions.

and constrained by the fit of the collinear mass spectrum to the data in the CRZ $\tau \tau$. A summary of the uncertainties and their impact on the best-fit LFV branching fraction is provided in Table 2, which shows that the sensitivity of the search is primarily limited by the available amount of data.

The best-fit expected and observed distributions of the combined NN output in the SR are shown in Fig. 2. The best-fit yields of $Z \rightarrow \tau \tau$ and events with fakes are close to the pre-fit predicted values and are determined with a relative precision of $2-4 \%$. Table 3 shows the best-fit expected background and signal yields and the observed number of events in the SR of the $e \tau$ and $\mu \tau$ channels with an additional requirement of a combined $\mathrm{NN}$ output $>0.7$ to consider the most signal-like events.

The best-fit amount of $Z \rightarrow \ell \tau$ signal corresponds to the branching fractions $\mathcal{B}(Z \rightarrow e \tau)=(-0.1 \pm 3.5$ (stat) \pm 2.3 (syst) $) \times 10^{-6}$ and $\mathcal{B}(Z \rightarrow \mu \tau)=(4.3 \pm 2.8$ (stat) $\pm 1.6($ syst $)) \times 10^{-6}$. The positive best-fit value of $\mathcal{B}(Z \rightarrow \mu \tau)$ is related to a small excess of observed events relative to the background-only hypothesis. This excess has a significance of 0.9 standard deviations when the events with $1 \mathrm{P}$ and $3 \mathrm{P} \tau_{\text {had-vis }}$ candidates are fitted simultaneously.

No statistically significant deviation from the SM prediction is observed, and upper limits on the LFV branching fractions are set. For the $\mu \tau$ channel, a more stringent upper limit is set by combining the likelihood function of the presented measurement and a similar measurement done with ATLAS Run 1 data $^{17}$. Systematic uncertainties from the two measurements are considered uncorrelated in the combined likelihood function. The upper limits are shown in Table 4 for LFV decays with different assumptions about the $\tau$-polarization state. In the scenario where the $\tau$ leptons are unpolarized, the observed upper limits at $95 \% \mathrm{CL}$ on $\mathcal{B}(Z \rightarrow e \tau)$ and $\mathcal{B}(Z \rightarrow \mu \tau)$ are $8.1 \times 10^{-6}$ and $9.5 \times 10^{-6}$, respectively.

In conclusion, these results from the ATLAS experiment at the LHC set stringent constraints on LFV Z-boson decays involving $\tau$ leptons (using only their hadronic decays), superseding the most stringent ones set by the LEP experiments more than two decades ago. The precision of these results is mainly limited by statistical uncertainties.

\section{Online content}

Any methods, additional references, Nature Research reporting summaries, source data, extended data, supplementary information, acknowledgements, peer review information; details of author contributions and competing interests; and statements of data and code availability are available at https://doi.org/10.1038/ s41567-021-01225-z.

Received: 7 October 2020; Accepted: 11 March 2021;

Published online: 1 July 2021

\section{References}

1. Glashow, S. Partial-symmetries of weak interactions. Nucl. Phys. 22, 579-588 (1961).

2. Weinberg, S. A model of leptons. Phys. Rev. Lett. 19, 1264-1266 (1967)

3. Salam, A. Weak and electromagnetic interactions. Conf. Proc. C 680519, 367-377 (1968).

4. 't Hooft, G. \& Veltman, M. Regularization and renormalization of gauge fields. Nucl. Phys. B 44, 189-213 (1972).

5. Super-Kamiokande Collaboration Evidence for oscillation of atmospheric neutrinos. Phys. Rev. Lett. 81, 1562-1567 (1998).

6. SNO Collaboration Direct evidence for neutrino flavor transformation from neutral-current interactions in the Sudbury Neutrino Observatory. Phys. Rev. Lett. 89, 011301 (2002).

7. Davidson, S., Lacroix, S. \& Verdier, P. LHC sensitivity to lepton flavour violating $Z$ boson decays. J. High Energy Phys. 9, 92-103 (2012).

8. Illana, J. I., Jack, M. \& Riemann, T. Predictions for $Z \rightarrow \tau \mu$ and related reactions. In Proc. 2nd Joint ECFA/DESY Workshop on Physics and Detectors for a Linear Electron Positron Collider 490-524 (DESY, 1999); https://arxiv. org/pdf/hep-ph/0001273.pdf

9. Illana, J. I. \& Riemann, T. Charged lepton flavor violation from massive neutrinos in $Z$ decays. Phys. Rev. D 63, 053004 (2001).

10. OPAL Collaboration A search for lepton flavor violating $Z^{0}$ decays. Z. Phys. C 67, 555-564 (1995).

11. DELPHI Collaboration Search for lepton flavor number violating $Z^{0}$ decays. Z. Phys. C 73, 243-251 (1997). 
12. ATLAS Collaboration The ATLAS experiment at the CERN Large Hadron Collider. J. Instrum. 3, S08003 (2008).

13. ATLAS Collaboration Search for lepton-flavor-violating decays of the $Z$ boson into a $\tau$ lepton and a light lepton with the ATLAS detector. Phys. Rev. D 98 092010 (2018).

14. ATLAS Collaboration ATLAS Insertable B-Layer Technical Design Report ATLAS-TDR-19; CERN-LHCC-2010-013 (CERN, 2010); https://cds.cern.ch/ record/1291633

15. Abbott, B. et al. Production and integration of the ATLAS insertable B-layer. J. Instrum. 13, T05008 (2018).

16. ATLAS Collaboration Performance of the ATLAS trigger system in 2015 Eur. Phys. J. C 77, 317-393 (2017).

17. ATLAS Collaboration Search for lepton-flavour-violating decays of the Higgs and $Z$ bosons with the ATLAS detector. Eur. Phys. J. C 77, 70-116 (2017).

18. ATLAS Collaboration Electron and photon performance measurements with the ATLAS detector using the 2015-2017 LHC proton-proton collision data. J. Instrum. 14, P12006 (2019).

19. ATLAS Collaboration Muon reconstruction performance of the ATLAS detector in proton-proton collision data at $\sqrt{s}=13 \mathrm{TeV}$. Eur. Phys. J. C 76, 292-337 (2016).

20. Cacciari, M., Salam, G. P. \& Soyez, G. The anti- $k_{t}$ jet clustering algorithm. J. High Energy Phys. 4, 63-75 (2008).

21. Cacciari, M., Salam, G. P. \& Soyez, G. FastJet user manual. Eur. Phys. J. C 72 , 1896-1965 (2012)

22. ATLAS Collaboration Jet energy scale and resolution measured in protonproton collisions at $\sqrt{s}=13 \mathrm{TeV}$ with the ATLAS detector. Preprint at https://arxiv.org/pdf/2007.02645.pdf (2020).

23. ATLAS Collaboration Identification of Hadronic Tau Lepton Decays using Neural Networks in the ATLAS Experiment ATL-PHYS-PUB-2019-033 (CERN, 2019); https://cds.cern.ch/record/2688062

24. ATLAS Collaboration Measurement of the Tau Lepton Reconstruction and Identification Performance in the ATLAS Experiment using pp Collisions at $\sqrt{s}=13 \mathrm{TeV}$ ATLAS-CONF-2017-029 (CERN, 2017); https://cds.cern.ch/ record/2261772

25. ATLAS Collaboration. ATLAS $b$-jet identification performance and efficiency measurement with $t \bar{t}$ events in $p p$ collisions at $t \bar{t} \mathrm{TeV}$. Eur. Phys. J. C 79, 970-1006 (2019).

26. ATLAS Collaboration Performance of missing transverse momentum reconstruction with the ATLAS detector using proton-proton collisions at $\sqrt{s}=13$ TeV. Eur. Phys. J. C 78, 903-969 (2018).

27. ATLAS Collaboration Performance in the ATLAS Detector using 2015-2016 LHC pp Collisions ATLAS-CONF-2018-023 (CERN, 2018); https://cds.cern. $\mathrm{ch} / \mathrm{record} / 2625233$

28. Sjöstrand, T. et al. An introduction to PYTHIA 8.2. Comput. Phys. Commun. 191, 159-177 (2015)

29. ATLAS Collaboration ATLAS Pythia 8 Tunes to 7-TeV Data ATL-PHYS-PUB-2014-021 (CERN, 2014); https://cds.cern.ch/record/1966419

30. Ball, R. D. et al. Parton distributions with LHC data. Nucl. Phys. B 867, 244-289 (2013).

31. Przedzinski, T., Richter-Was, E. \& Was, Z. Documentation of TauSpinner algorithms: program for simulating spin effects in $\tau$-lepton production at LHC. Eur. Phys. J. C 79, 91-113 (2019).

32. Bothmann, E. et al. Event generation with Sherpa 2.2. SciPost Phys. 7 34-73 (2019).
33. Ball, R. D. et al. Parton distributions for the LHC run II. J. High Energy Phys. 4, 40-191 (2015).

34. Gleisberg, T. \& Höche, S. Comix, a new matrix element generator. J. High Energy Phys. 12, 39-64 (2008).

35. Buccioni, F. et al. OpenLoops 2. Eur. Phys. J. C 79, 866-946 (2019).

36. Cascioli, F., Maierhöfer, P. \& Pozzorini, S. Scattering amplitudes with open loops. Phys. Rev. Lett. 108, 111601 (2012).

37. Denner, A., Dittmaier, S. \& Hofer, L. Collier: a Fortran-based complex one-loop library in extended regularizations. Comput. Phys. Commun. 212 , 220-238 (2017)

38. Schumann, S. \& Krauss, F. A Parton shower algorithm based on CataniSeymour dipole factorisation. J. High Energy Phys. 3, 38-97 (2008).

39. Höche, S., Krauss, F., Schönherr, M. \& Siegert, F. A critical appraisal of NLO + PS matching methods. J. High Energy Phys. 9, 49-83 (2012).

40. Höche, S., Krauss, F., Schönherr, M. \& Siegert, F. QCD matrix elements + parton showers. The NLO case. J. High Energy Phys. 4, 27-41 (2013).

41. Catani, S., Krauss, F., Kuhn, R. \& Webber, B. R. QCD matrix elements + parton showers. J. High Energy Phys. 11, 63-84 (2001).

42. Höche, S., Krauss, F., Schumann, S. \& Siegert, F. QCD matrix elements and truncated showers. J. High Energy Phys. 5, 53-94 (2009).

43. Alioli, S., Nason, P., Oleari, C. \& Re, E. A general framework for implementing NLO calculations in shower Monte Carlo programs: the POWHEG BOX. J. High Energy Phys. 6, 43-99 (2010).

44. Agostinelli, S. et al. Geant4-a simulation toolkit. Nucl. Instrum. Methods Phys. Res. A 506, 250-303 (2003).

45. ATLAS Collaboration The Pythia 8 A3 Tune Description of ATLAS Minimum Bias and Inelastic Measurements Incorporating the Donnachie-Landshoff Diffractive Model ATL-PHYS-PUB-2016-017 (CERN, 2016); https://cds.cern. ch/record/2206965

46. ATLAS Collaboration Measurement of the transverse momentum distribution of Drell-Yan lepton pairs in proton-proton collisions at $\sqrt{s}=13 \mathrm{TeV}$ with the ATLAS detector. Eur. Phys. J. C 80, 616-644 (2020).

47. ATLAS Collaboration Measurement of $W^{ \pm}$and $Z$-boson production cross-sections in $p p$ collisions at $\sqrt{s}=13 \mathrm{TeV}$ with the ATLAS detector. Phys. Lett. B 759, 601-621 (2016).

48. Read, A. L. Presentation of search results: the $\mathrm{CL}_{\mathrm{s}}$ technique. J. Phys. G 28, 2693-2704 (2002)

Publisher's note Springer Nature remains neutral with regard to jurisdictional claims in published maps and institutional affiliations.

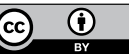

Open Access This article is licensed under a Creative Commons Attribution 4.0 International License, which permits use, sharing, adaptation, distribution and reproduction in any medium or format, as long as you give appropriate credit to the original author(s) and the source, provide a link to the Creative Commons license, and indicate if changes were made. The images or other third party material in this article are included in the article's Creative Commons license, unless indicated otherwise in a credit line to the material. If material is not included in the article's Creative Commons license and your intended use is not permitted by statutory regulation or exceeds the permitted use, you will need to obtain permission directly from the copyright holder. To view a copy of this license, visit http://creativecommons.org/licenses/by/4.0/.

(C) The Author(s) 2021 


\section{Methods}

Neural network classifiers. Several binary NN classifiers are trained for both the $e \tau$ and $\mu \tau$ channels to discriminate signal from the three major backgrounds: $W+$ jets, $Z \rightarrow \tau \tau$ and $Z \rightarrow \ell \ell$. They are referred to as $\mathrm{NN}_{\mathrm{Wjets}}$, $\mathrm{NN}_{\mathrm{Z} \tau}$ and $\mathrm{NN}_{\mathrm{Z} \ell \ell}$, respectively.

The NNs are trained using simulated events selected with the same criteria as those used in the SR, except that the cuts on $m_{\mathrm{vis}}(\ell, \tau)$ and the NN output are omitted, and real $\tau_{\text {had-vis }}$ candidates from $Z \rightarrow \ell \tau$ and $Z \rightarrow \tau \tau$ are required to pass less stringent identification criteria so as to increase the training sample size. For the $Z \rightarrow \ell \ell$ process, only events where the $\tau_{\text {had-vis }}$ candidate is a misidentified light lepton are used. For the $W+$ jets process, jets misidentified as $\tau_{\text {had-vis }}$ are modelled by simulations. Different NNs are trained separately for $e \tau$ and $\mu \tau$ events as well as for events with $1 \mathrm{P}$ or $3 \mathrm{P} \tau_{\text {had-vis }}$ candidates. To increase the signal sample size, the $Z \rightarrow e \tau$ and $Z \rightarrow \mu \tau$ samples are combined and used for training in both channels, assuming equivalent event topology when exchanging $e$ and $\mu$. Owing to the low expected yield of $Z \rightarrow \ell \ell$ events with $3 \mathrm{P} \tau_{\text {had-vis }}$ candidates, no classifier is trained to discriminate them from background.

A mixture of low-level and high-level kinematic variables are used as input to the NNs. The low-level variables include the four-momenta of the reconstructed $\ell$ (refs. ${ }^{18,19}$ ), $\tau_{\text {had } \text { vis }}$ candidate ${ }^{23,24}$ and $E_{\mathrm{T}}^{\text {miss }}$ (refs. ${ }^{26,27}$ ). To remove known spatial symmetries for optimal training, the low-level variables are transformed in a way that preserves the Lorentz invariance before they are fed into the NNs. The transformation consists of the following steps: first, the $\ell-\tau_{\text {had-vis }}-E_{\mathrm{T}}^{\mathrm{miss}}$ system is boosted in a direction in the plane transverse to the beam line such that the total transverse momentum of the system is zero; the system is then rotated about the $z$ axis such that the direction of $E_{\mathrm{T}}^{\text {miss }}$ is aligned with the $x$ axis; if the $\tau_{\text {had-vis }}$ candidate's momentum has a negative $z$ component, the entire system is rotated about the new $x$ axis by $180^{\circ}$. After the transformation, only six independent non-vanishing components are left (the $\tau_{\text {had-vis }}$ candidate is assumed to have zero rest mass), which are the inputs to the NNs.

The high-level variables include $\Delta \alpha$, which is a kinematic discriminant defined $^{7}$ as

$$
\Delta \alpha=\frac{m_{Z}^{2}-m_{\tau}^{2}}{2 p(\ell) \times p\left(\tau_{\text {had }- \text { vis }}\right)}-\frac{p_{\mathrm{T}}(\ell)}{p_{\mathrm{T}}\left(\tau_{\text {had }- \text { vis }}\right)}
$$

where $m_{Z}$ and $m_{\tau}$ are the nominal masses of the $Z$ boson and $\tau$ lepton, respectively, and $p$ denotes four-momentum. It is specifically defined to test the assumptions that the missing momentum of the event is collinear with the $\tau_{\text {had-vis }}$ candidate, and that the $\tau$ and light leptons in the event are decay products of an on-shell $Z$ boson. For a signal event, where these assumptions are approximately true, it is expected that $\Delta \alpha \approx 0$. Meanwhile, for an SM background event, the value is expected to deviate from zero in general. The other high-level variables are the invariant mass of the $\ell-\tau_{\text {had-vis }}$ system, the collinear mass $m_{\text {coll }}(\ell, \tau)$ and the invariant mass of the light lepton and the track associated with the $\tau_{\text {had-vis }}$ candidate (only used by the $Z \rightarrow \ell \ell$ classifier).

The training and optimization of the NN classifiers are performed using the open-source software package KERAS ${ }^{49}$. All of the NNs used in the analysis share the same architecture. Each NN consists of an input layer, two hidden layers of 20 nodes each, and an output layer with a single node. Each layer is fully connected to the neighbouring layers. Low-level and high-level variables are treated in the same way in the input layer. The hidden-layer nodes use rectified linear activation functions, while the output node uses a sigmoid activation function. The NNs are trained using the Adam algorithm ${ }^{50}$ to optimize the binary cross entropy. All the NNs are trained with a batch size of 256 and 200 epochs. The number of hidden layers, the number of nodes per layer, the training batch size and the learning rate parameter of the optimizer are simultaneously chosen by maximizing the area under the expected receiver operating characteristic curve. The optimization is done with a grid scan. No regularization or dropout is added, and no sign of overtraining is observed. For other configurations and hyperparameters that have not been mentioned, the default settings in KERAS 1.1.0 are used.

Each NN classifier outputs a score between 0 and 1 for each event, where a higher score indicates that the event is more signal-like. The output scores from the different classifiers are combined into the final discriminant (combined NN output) using the formula

$$
\text { Combined NN output }=1-\sqrt{\frac{\sum_{b} w_{b} \times\left(1-\mathrm{NN}_{b} \text { output }\right)^{2}}{\sum_{b} w_{b}}}
$$

where $b=W$ jets, $Z \tau \tau, Z \ell \ell$ and $w_{b}$ are constant parameters. Output scores for events with $1 \mathrm{P} \tau_{\text {had-vis }}$ candidates and those with $3 \mathrm{P} \tau_{\text {had-vis }}$ candidates are combined separately. The summation is over $W$ jets, $Z \tau \tau$ and $Z \ell \ell$ for events with $1 \mathrm{P} \tau_{\text {had-vis }}$ candidates, and only over $W$ jets and $Z \tau \tau$ for events with $3 \mathrm{P} \tau_{\text {had-vis }}$ candidates.

By construction, the combined $\mathrm{NN}$ output ranges between 0 and 1 , where 0 represents the most background-like (and 1 the most signal-like) event possible. The choice of values of $w_{b}$ affects the expected sensitivity of the analysis because they change how events from the different background processes are distributed along the range of combined NN output values, and thus impact the ability of the binned maximum-likelihood fit to determine the background contributions. The values of $w_{b}$ are chosen with a grid scan to minimize the expected upper limit on the branching fraction in the absence of a signal. The chosen values have the ratio $w_{Z \pi \tau}: w_{\text {Wets }}: w_{z e \ell}=1.0: 1.5: 0.33$. As could be expected, the optimized weights loosely reflect the impact of the uncertainties in the corresponding backgrounds on the determination of the signal branching fraction.

Maximum-likelihood fit. Binned maximum-likelihood fits are implemented using the statistical analysis packages ROOFIT ${ }^{51}$, ROOSTATS ${ }^{52}$ and HISTFITTER ${ }^{53}$. The expected binned distributions of the combined NN output in the SR and the collinear mass in the CRZ $\tau \tau$ are fit to data to extract evidence of signal events. Fitting the data in the CRZ $\tau \tau$ and in part of the SR with low combined $\mathrm{NN}$ output values (where no signal is expected) benefits the overall sensitivity to the signal, because it reduces the uncertainties of the background model in the high combined NN output value region, where most of the signal is expected. Owing to the differences in background composition, acceptance and efficiencies, regions with $1 \mathrm{P}$ and $3 \mathrm{P} \tau_{\text {had-vis }}$ candidates are fit separately but simultaneously. The probabilities of compatibility between the data and the background-only or background-plus-signal hypotheses are assessed using the modified frequentist $\mathrm{CL}_{\mathrm{S}}$ method $^{48}$, and exclusion upper limits on $\mathcal{B}(Z \rightarrow \ell \tau)$ are set by the inversion of these hypothesis tests.

The background-plus-signal model has four unconstrained parameters before the fit. Two of the parameters determine the overall yields of events with $1 \mathrm{P}$ and $3 \mathrm{P}$ fakes separately. A third parameter determines $\sigma_{Z}$ times the overall acceptance and reconstruction efficiency of events with a true $\ell \tau_{\text {had-vis }}$ final state. It is applied to the normalizations of both the signal and $Z \rightarrow \tau \tau$ events to ensure that the same $\sigma_{Z}$ times acceptance is estimated for both processes. The last unconstrained parameter is the parameter of interest $\mu_{\mathrm{sig}}$, which controls the normalization of signal events. Given the similarity between the signal and $Z \rightarrow \tau \tau \rightarrow \ell \tau_{\text {had-vis }}+3 \nu$ final states and that both processes are estimated with the same $\sigma_{Z}$ and acceptance and efficiency corrections, this choice of parameterization reduces the impact on the determined $\mathcal{B}(Z \rightarrow \ell \tau)$ from detector effects and uncertainties in predicting $\sigma_{Z}$. The parameter of interest represents

$$
\mu_{\text {sig }}=\frac{\mathcal{B}(Z \rightarrow \ell \tau)}{\mathcal{B}_{\text {pre-fit }}(Z \rightarrow \ell \tau)}
$$

where $\mathcal{B}_{\text {pre-fit }}(Z \rightarrow \ell \tau)$ is an arbitrary branching fraction to which the signal prediction is normalized. Although the physical branching fraction must be positive, the parameter of interest in the fit is not constrained to be positive.

Systematic uncertainties are represented by nuisance parameters (NPs) with Gaussian constraints in the likelihood function. The impact of uncertainties on both the shape and normalization of the predicted distributions are taken into account. Uncertainties in the energy calibration and resolution as well as in the trigger, reconstruction, identification and isolation efficiencies of jets, electrons, muons, $\tau_{\text {had-vis }}$ and $E_{\mathrm{T}}^{\text {miss }}$ are considered. Theoretical uncertainties in the production cross-sections affect only the predictions of the minor backgrounds, because the $Z \rightarrow \tau \tau$ and signal yields are determined in the maximum-likelihood fit to data and the $Z \rightarrow \ell \ell$ yield is determined by the measured value of $\sigma_{Z}$. Statistical uncertainties in the determination of the fake factors are also considered. They are modelled by one NP per $p_{\mathrm{T}}$ bin in which the fake factors are measured. As noted in the section 'Constraints on $\mathcal{B}(Z \rightarrow \ell \tau)$ ' the dominant uncertainties in the analysis are the statistical uncertainties in determining how many events have fakes and the systematic uncertainties in the reconstructed $\tau_{\text {had-vis }}$ energy.

For the $\mu \tau$ channel, the likelihood functions of the presented measurement and of the measurement in ref. ${ }^{17}$ are combined. As the two measurements are statistically uncorrelated and the predictions are based on different methods, NPs in the individual likelihood functions are considered uncorrelated in the combination. The method of combination is the same as in ref. ${ }^{13}$.

\section{Data availability}

The experimental data that support the findings of this study are available in HEPData with the identifier https://www.hepdata.net/record/96390. The ATLAS software is available at the following link: https://gitlab.cern.ch/atlas/athena.

\section{References}

49. Chollet, F. et al. Keras (Keras, 2015); https://keras.io

50. Kingma, D. P. \& Ba, J. Adam: a method for stochastic optimization. Preprint at https://arxiv.org/pdf/1412.6980.pdf (2014).

51. Verkerke, W. \& Kirkby, D. P. The RooFit toolkit for data modeling. Preprint at https://arxiv.org/pdf/physics/0306116.pdf (2003).

52. Moneta, L. et al. The RooStats Project. Preprint at https://arxiv.org/ pdf/1009.1003.pdf (2010).

53. Baak, M. et al. HistFitter software framework for statistical data analysis. Eur Phys. J. C 75, 153-173 (2015).

54. ATLAS Collaboration ATLAS Computing Acknowledgments ATL-SOFT-PUB-2020-001 (CERN, 2020); https://cds.cern.ch/record/2717821 


\section{Acknowledgements}

We acknowledge our late colleague, Olga Igonkina (1973-2019), for inspiring and driving this and other searches for lepton flavour violation within the ATLAS experiment. Her curiosity and intelligence remain an inspiration to the ATLAS Collaboration. We thank CERN for the very successful operation of the LHC, as well as the support staff from our institutions, without whom ATLAS could not be operated efficiently. We acknowledge the support of ANPCyT, Argentina; YerPhI, Armenia; ARC, Australia; BMWFW and FWF, Austria; ANAS, Azerbaijan; SSTC, Belarus; CNPq and FAPESP, Brazil; NSERC, NRC and CFI, Canada; CERN; ANID, Chile; CAS, MOST and NSFC, China; COLCIENCIAS, Colombia; MSMT CR, MPO CR and VSC CR, Czech Republic; DNRF and DNSRC, Denmark; IN2P3-CNRS and CEA-DRF/IRFU, France; SRNSFG, Georgia; BMBF, HGF and MPG, Germany; GSRT, Greece; RGC and Hong Kong SAR, China; ISF and Benoziyo Center, Israel; INFN, Italy; MEXT and JSPS, Japan; CNRST, Morocco; NWO, Netherlands; RCN, Norway; MNiSW and NCN, Poland; FCT, Portugal; MNE/IFA, Romania; MES of Russia and NRC KI, Russia Federation; JINR; MESTD, Serbia; MSSR, Slovakia; ARRS and MIZŠ, Slovenia; DST/NRF, South Africa; MICINN, Spain; SRC and Wallenberg Foundation, Sweden; SERI, SNSF and Cantons of Bern and Geneva, Switzerland; MOST, Taiwan; TAEK, Turkey; STFC, UK; DOE and NSF, United States. In addition, individual groups and members have received support from BCKDF, CANARIE, Compute Canada and CRC, Canada; ERC, ERDF, Horizon 2020, Marie Skłodowska-Curie Actions and COST, EU; Investissements d'Avenir Labex, Investissements d'Avenir Idex and ANR, France; DFG and AvH Foundation, Germany; Herakleitos, Thales and Aristeia programmes co-financed by EU-ESF and the Greek NSRF, Greece; BSF-NSF and GIF, Israel; La Caixa Banking Foundation, CERCA Programme Generalitat de Catalunya and PROMETEO and GenT Programmes Generalitat Valenciana, Spain; Göran Gustafssons Stiftelse, Sweden; The Royal Society and Leverhulme Trust, UK. The crucial computing support from all WLCG partners is acknowledged gratefully, in particular from CERN, the ATLAS Tier-1 facilities at TRIUMF (Canada), NDGF (Denmark, Norway, Sweden), CC-IN2P3 (France), KIT/GridKA (Germany), INFN-CNAF (Italy), NL-T1 (Netherlands), PIC (Spain), ASGC (Taiwan), RAL (UK) and BNL (USA), the Tier-2 facilities worldwide and large non-WLCG resource providers. Major contributors of computing resources are listed in ref. ${ }^{54}$.

\section{Author contributions}

All authors contributed to the publication, being variously involved in the design and the construction of the detectors, in writing software, calibrating subsystems, operating the detectors and acquiring data, and finally analysing the processed data. The ATLAS Collaboration members discussed and approved the scientific results. The manuscript was prepared by a subgroup of authors appointed by the collaboration and subject to an internal collaboration-wide review process. All authors reviewed and approved the final version of the manuscript.

\section{Competing interests}

The authors declare no competing interests.

\section{Additional information}

Extended data is available for this paper at https://doi.org/10.1038/s41567-021-01225-z. Supplementary information The online version contains supplementary material available at https://doi.org/10.1038/s41567-021-01225-z.

Correspondence and requests for materials should be addressed to G.A.

Peer review information Nature Physics thanks Michael Schmidt, Roger Wolf and Scott Yost for their contribution to the peer review of this work.

Reprints and permissions information is available at www.nature.com/reprints. 


\section{ATLAS Collaboration}

G. Aad ${ }^{1 凶}$, B. Abbott' ${ }^{2}$, D. C. Abbott ${ }^{3}$, A. Abed Abud ${ }^{4}$, K. Abeling ${ }^{5}$, D. K. Abhayasinghe ${ }^{6}$, S. H. Abidi?,

O. S. Abou Zeid ${ }^{8}$, N. L. Abraham9, H. Abramowicz' ${ }^{10}$, H. Abreu"11, Y. Abulaiti"12, B. S. Acharya ${ }^{13,14,244}$,

B. Achkar ${ }^{5}$, L. Adam ${ }^{15}$, C. Adam Bourdarios ${ }^{16}$, L. Adamczyk ${ }^{17}$, L. Adamek, J. Adelman ${ }^{18}$,

A. Adiguzel ${ }^{19,245}$, S. Adorni ${ }^{20}$, T. Adye ${ }^{21}$, A. A. Affolder ${ }^{22}$, Y. Afik ${ }^{11}$, C. Agapopoulou ${ }^{23}$, M. N. Agaras ${ }^{24}$,

A. Aggarwal' ${ }^{25}$, C. Agheorghiesei ${ }^{26}$, J. A. Aguilar-Saavedra27,28,29,246, A. Ahmad ${ }^{4}$, F. Ahmadov ${ }^{30}$,

W. S. Ahmed ${ }^{31}$, X. Ai ${ }^{32}$, G. Aielli33,34, S. Akatsuka ${ }^{35}$, M. Akbiyik ${ }^{15}$, T. P. A. Åkesson ${ }^{36}$, E. Akilli20,

A. V. Akimov ${ }^{37}$, K. Al Khoury ${ }^{23}$, G. L. Alberghi ${ }^{38,39}$, J. Albert ${ }^{40}$, M. J. Alconada Verzini ${ }^{10}$,

S. Alderweireldt' ${ }^{4}$ M. Aleksa ${ }^{4}$, I. N. Aleksandrov ${ }^{30}$, C. Alexa ${ }^{41}$, T. Alexopoulos ${ }^{42}$, A. Alfonsi ${ }^{43}$,

F. Alfonsi ${ }^{38,39}$, M. Alhroob ${ }^{2}$, B. Ali ${ }^{44}$, S. Ali ${ }^{45}$, M. Aliev ${ }^{46}$, G. Alimonti ${ }^{47}$, C. Allaire ${ }^{4}$, B. M. M. Allbrooke ${ }^{9}$,

B. W. Allen ${ }^{48}$, P. P. Allport ${ }^{49}$, A. Aloisio ${ }^{50,51}$, F. Alonso ${ }^{52}$, C. Alpigiani ${ }^{53}$, E. Alunno Camelia ${ }^{33,34}$,

M. Alvarez Estevez ${ }^{54}$, M. G. Alviggi ${ }^{50,51}$, Y. Amaral Coutinho55, A. Ambler ${ }^{31}$, L. Ambroz ${ }^{56}$, C. Amelung ${ }^{4}$,

D. Amidei ${ }^{57}$, S. P. Amor Dos Santos ${ }^{27}$, S. Amoroso ${ }^{58}$, C. S. Amrouche ${ }^{20}$, F. An ${ }^{59}$, C. Anastopoulos ${ }^{60}$,

N. Andari ${ }^{61}$, T. Andeen ${ }^{62}$, J. K. Anders ${ }^{63}$, S. Y. Andrean ${ }^{64,65}$, A. Andreazza ${ }^{47,66}$, V. Andrei ${ }^{67}$, C. R. Anelli ${ }^{40}$,

S. Angelidakis ${ }^{68}$, A. Angerami ${ }^{69}$, A. V. Anisenkov ${ }^{70,71}$, A. Annovi ${ }^{72}$, C. Antel ${ }^{20}$, M. T. Anthony ${ }^{60}$,

E. Antipov ${ }^{73}$, M. Antonelli ${ }^{74}$, D. J. A. Antrim ${ }^{32}$, F. Anulli ${ }^{75}$, M. Aoki ${ }^{76}$, J. A. Aparisi Pozo ${ }^{77}$, M. A. Aparo9,

L. Aperio Bella ${ }^{58}$, N. Aranzabal ${ }^{4}$, V. Araujo Ferraz ${ }^{78}$, R. Araujo Pereira ${ }^{55}$, C. Arcangeletti ${ }^{74}$,

A. T. H. Arce ${ }^{79}$, J-F. Arguin ${ }^{80}$, S. Argyropoulos ${ }^{81}$, J.-H. Arling ${ }^{58}$, A. J. Armbruster 4 , A. Armstrong ${ }^{82}$,

O. Arnaez , H. Arnold ${ }^{43}$, Z. P. Arrubarrena Tame ${ }^{83}$, G. Artoni ${ }^{56}$, H. Asada ${ }^{84}$, K. Asai ${ }^{85}$, S. Asai ${ }^{86}$,

T. Asawatavonvanich ${ }^{87}$, N. Asbah ${ }^{88}$, E. M. Asimakopoulou ${ }^{89}$, L. Asquith ${ }^{9}$, J. Assahsah' ${ }^{90}$,

K. Assamagan91, R. Astalos ${ }^{92}$, R. J. Atkin ${ }^{93,94}$, M. Atkinson ${ }^{95}$, N. B. Atlay ${ }^{96}$, H. Atmani ${ }^{23}$,

P. A. Atmasiddha ${ }^{57}$, K. Augsten ${ }^{44}$, V. A. Austrup ${ }^{97}$, G. Avolio ${ }^{4}$, M. K. Ayoub ${ }^{98}$, G. Azuelos ${ }^{80,247}$,

D. Babal ${ }^{92}$, H. Bachacou ${ }^{61}$, K. Bachas ${ }^{99}$, F. Backman ${ }^{64,65}$, P. Bagnaia ${ }^{75,100}$, M. Bahmani ${ }^{101}$,

H. Bahrasemani ${ }^{102}$, A. J. Bailey ${ }^{77}$, V. R. Bailey ${ }^{95}$, J. T. Baines ${ }^{21}$, C. Bakalis ${ }^{42}$, O. K. Baker ${ }^{103}$, P. J. Bakker ${ }^{43}$,

E. Bakos ${ }^{104}$, D. Bakshi Gupta ${ }^{105}$, S. Balaji ${ }^{106}$, R. Balasubramanian ${ }^{43}$, E. M. Baldin ${ }^{70,71}$, P. Balek ${ }^{107}$, F. Balli61,

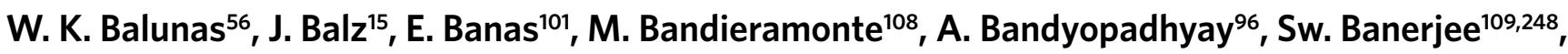

L. Barak ${ }^{10}$, W. M. Barbe ${ }^{24}$, E. L. Barberio ${ }^{110}$, D. Barberis ${ }^{111,112}$, M. Barbero', G. Barbour ${ }^{113}$, T. Barillari ${ }^{114}$,

M-S. Barisits ${ }^{4}$, J. Barkeloo48, T. Barklow ${ }^{115}$, R. Barnea11, B. M. Barnett'21, R. M. Barnett ${ }^{32}$,

Z. Barnovska-Blenessy ${ }^{116}$, A. Baroncelli1"16, G. Barone ${ }^{91}$, A. J. Barr ${ }^{56}$, L. Barranco Navarro ${ }^{64,65}$,

F. Barreiro ${ }^{54}$, J. Barreiro Guimarães da Costa ${ }^{98}$, U. Barron ${ }^{10}$, S. Barsov ${ }^{117}$, F. Bartels ${ }^{67}$, R. Bartoldus ${ }^{115}$,

G. Bartolini', A. E. Barton ${ }^{118}$, P. Bartos ${ }^{92}$, A. Basalaev ${ }^{58}$, A. Basan ${ }^{15}$, A. Bassalat ${ }^{23,249}$, M. J. Basso7,

R. L. Bates ${ }^{119}$, S. Batlamous ${ }^{120}$, J. R. Batley ${ }^{121}$, B. Batool ${ }^{122}$, M. Battaglia22, M. Bauce ${ }^{75,100}$, F. Bauer ${ }^{61}$,

P. Bauer ${ }^{123}$, H. S. Bawa ${ }^{124}$, A. Bayirli' ${ }^{19}$, J. B. Beacham ${ }^{79}$, T. Beau ${ }^{125}$, P. H. Beauchemin ${ }^{126}$, F. Becherer ${ }^{81}$,

P. Bechtle ${ }^{123}$, H. C. Beck ${ }^{5}$, H. P. Beck ${ }^{63,250}$, K. Becker ${ }^{127}$, C. Becot ${ }^{58}$, A. Beddall ${ }^{128}$, A. J. Beddall ${ }^{129}$,

V. A. Bednyakov ${ }^{30}$, M. Bedognetti ${ }^{43}$, C. P. Bee ${ }^{130}$, T. A. Beermann ${ }^{97}$, M. Begalli5, M. Begel ${ }^{91}$,

A. Behera ${ }^{130}$, J. K. Behr ${ }^{58}$, F. Beisiegel ${ }^{123}$, M. Belfkir ${ }^{16}$, A. S. Bell ${ }^{113}$, G. Bella ${ }^{10}$, L. Bellagamba ${ }^{39}$,

A. Bellerive ${ }^{131}$, P. Bellos ${ }^{68}$, K. Beloborodov ${ }^{70,71}$, K. Belotskiy ${ }^{132}$, N. L. Belyaev ${ }^{132}$, D. Benchekroun ${ }^{133}$,

N. Benekos ${ }^{42}$, Y. Benhammou ${ }^{10}$, D. P. Benjamin ${ }^{12}$, M. Benoit ${ }^{91}$, J. R. Bensinger ${ }^{134}$, S. Bentvelsen ${ }^{43}$,

L. Beresford ${ }^{56}$, M. Beretta74, D. Berge ${ }^{96}$, E. Bergeaas Kuutmann ${ }^{89}$, N. Berger ${ }^{16}$, B. Bergmann ${ }^{44}$,

L. J. Bergsten ${ }^{134}$, J. Beringer ${ }^{32}$, S. Berlendis ${ }^{135}$, G. Bernardi ${ }^{125}$, C. Bernius ${ }^{115}$, F. U. Bernlochner ${ }^{123}$,

T. Berry ${ }^{6}$, P. Berta ${ }^{15}$, A. Berthold ${ }^{136}$, I. A. Bertram ${ }^{118}$, O. Bessidskaia Bylund ${ }^{97}$, N. Besson ${ }^{61}$, S. Bethke ${ }^{114}$,

A. Betti ${ }^{137}$, A. J. Bevan ${ }^{138}$, J. Beyer ${ }^{114}$, S. Bhatta ${ }^{130}$, D. S. Bhattacharya ${ }^{139}$, P. Bhattarai ${ }^{134}$, V. S. Bhopatkar ${ }^{12}$, 
R. Bi ${ }^{108}$, R. M. Bianchi ${ }^{108}$, O. Biebel ${ }^{83}$, D. Biedermann ${ }^{96}$, R. Bielski ${ }^{4}$, K. Bierwagen ${ }^{15}$, N. V. Biesuz ${ }^{72,140}$, M. Biglietti ${ }^{141}$, T. R. V. Billoud ${ }^{44}$, M. Bindi ${ }^{5}$, A. Bingul ${ }^{128}$, C. Bini ${ }^{75,100}$, S. Biondi ${ }^{38,39}$, C. J. Birch-sykes ${ }^{142}$, M. Birman ${ }^{107}$, T. Bisanz ${ }^{4}$, J. P. Biswal ${ }^{143}$, D. Biswas ${ }^{109,248}$, A. Bitadze ${ }^{142}$, C. Bittrich ${ }^{136}$, K. Bjørke $^{144}$, T. Blazek ${ }^{92}$, I. Bloch ${ }^{58}$, C. Blocker ${ }^{134}$, A. Blue ${ }^{119}$, U. Blumenschein ${ }^{138}$, G. J. Bobbink ${ }^{43}$,

V. S. Bobrovnikov70,71, S. S. Bocchetta ${ }^{36}$, D. Bogavac ${ }^{145}$, A. G. Bogdanchikov70,71, C. Bohm ${ }^{64}$, V. Boisvert ${ }^{6}$, P. Bokan ${ }^{5,89}$, T. Bold ${ }^{17}$, A. E. Bolz ${ }^{146}$, M. Bomben ${ }^{125}$, M. Bona ${ }^{138}$, J. S. Bonilla ${ }^{48}$, M. Boonekamp ${ }^{61}$, C. D. Booth ${ }^{6}$, A. G. Borbély ${ }^{119}$, H. M. Borecka-Bielska147, L. S. Borgna ${ }^{113}$, A. Borisov ${ }^{148}$, G. Borissov ${ }^{118}$, D. Bortoletto ${ }^{56}$, D. Boscherini ${ }^{39}$, M. Bosman ${ }^{145}$, J. D. Bossio Sola ${ }^{31}$, K. Bouaouda ${ }^{133}$, J. Boudreau ${ }^{108}$, E. V. Bouhova-Thacker ${ }^{118}$, D. Boumediene ${ }^{24}$, A. Boveia' ${ }^{149}$, J. Boyd ${ }^{4}$, D. Boye ${ }^{150,151}$, I. R. Boyko ${ }^{30}$, A. J. Bozson ${ }^{6}$, J. Bracinik ${ }^{49}$, N. Brahimi ${ }^{152}$, G. Brandt ${ }^{97}$, O. Brandt ${ }^{121}$, F. Braren ${ }^{58}$, B. Brau ${ }^{3}$, J. E. Brau ${ }^{48}$, W. D. Breaden Madden ${ }^{119}$, K. Brendlinger ${ }^{58}$, R. Brener ${ }^{11}$, L. Brenner ${ }^{4}$, R. Brenner ${ }^{89}$, S. Bressler ${ }^{107}$, B. Brickwedde ${ }^{15}$, D. L. Briglin ${ }^{49}$, D. Britton ${ }^{119}$, D. Britzger ${ }^{114}$, I. Brock ${ }^{123}$, R. Brock ${ }^{153}$, G. Brooijmans ${ }^{69}$, W. K. Brooks ${ }^{154}$, E. Brost ${ }^{91}$, P. A. Bruckman de Renstrom ${ }^{101}$, B. Brüers ${ }^{58}$, D. Bruncko ${ }^{155}$, A. Bruni ${ }^{39}$, G. Bruni ${ }^{39}$, M. Bruschi ${ }^{39}$, N. Bruscino ${ }^{75,100}$, L. Bryngemark ${ }^{115}$, T. Buanes ${ }^{156}$, Q. Buat ${ }^{130}$, P. Buchholz ${ }^{122}$, A. G. Buckley ${ }^{119}$, I. A. Budagov ${ }^{30}$, M. K. Bugge ${ }^{144}$, O. Bulekov ${ }^{132}$, B. A. Bullard ${ }^{88}$, T. J. Burch ${ }^{18}$, S. Burdin ${ }^{147}$, C. D. Burgard ${ }^{43}$, A. M. Burger73, B. Burghgrave ${ }^{105}$, J. T. P. Burr ${ }^{58}$, C. D. Burton ${ }^{62}$, J. C. Burzynski ${ }^{3}$, V. Büscher ${ }^{15}$, E. Buschmann ${ }^{5}$, P. J. Bussey ${ }^{119}$, J. M. Butler ${ }^{157}$, C. M. Buttar ${ }^{119}$, J. M. Butterworth ${ }^{113}$, P. Butti ${ }^{4}$, W. Buttinger ${ }^{21}$, C. J. Buxo Vazquez ${ }^{153}$, A. Buzatu ${ }^{45}$, A. R. Buzykaev ${ }^{70,71}$, G. Cabras ${ }^{38,39}$, S. Cabrera Urbánn 77 , D. Caforio ${ }^{158}$, H. Cai ${ }^{108}$, V. M. M. Cairo ${ }^{115}$, O. Cakir ${ }^{159}$, N. Calace ${ }^{4}$, P. Calafiura ${ }^{32}$, G. Calderini ${ }^{125}$, P. Calfayan ${ }^{160}$, G. Callea ${ }^{119}$, L. P. Caloba ${ }^{55}$, A. Caltabiano ${ }^{33,34}$, S. Calvente Lopez ${ }^{54}$, D. Calvet ${ }^{24}$, S. Calvet ${ }^{24}$, T. P. Calvet ${ }^{1}$, M. Calvetti ${ }^{72,140}$, R. Camacho Toro ${ }^{125}$, S. Camarda ${ }^{4}$, D. Camarero Munoz ${ }^{54}$, P. Camarri ${ }^{33,34}$, M. T. Camerlingo ${ }^{141,161}$, D. Cameron ${ }^{144}$, C. Camincher ${ }^{4}$, S. Campana ${ }^{4}$, M. Campanelli ${ }^{113}$, A. Camplani ${ }^{8}$, V. Canale ${ }^{50,51}$, A. Canesse ${ }^{31}$, M. Cano Bret ${ }^{162}$, J. Cantero ${ }^{73}$, T. Cao ${ }^{10}$, Y. Cao ${ }^{95}$, M. Capua ${ }^{163,164}$, R. Cardarelli;3, F. Cardillo77, G. Carducci'163,164, I. Carli ${ }^{165}$, T. Carli ${ }^{4}$, G. Carlino ${ }^{50}$, B. T. Carlson ${ }^{108}$, E. M. Carlson ${ }^{40,166}$, L. Carminati ${ }^{47,66}$, R. M. D. Carney ${ }^{115}$, S. Caron ${ }^{25}$, E. Carquin ${ }^{154}$, S. Carrá ${ }^{58}$, G. Carratta ${ }^{38,39}$, J. W. S. Carter7, T. M. Carter ${ }^{167}$, M. P. Casado ${ }^{145,251}$, A. F. Casha7, E. G. Castiglia ${ }^{103}$, F. L. Castillo77, L. Castillo Garcia'145, V. Castillo Gimenez ${ }^{77}$, N. F. Castro ${ }^{27,168}$, A. Catinaccio ${ }^{4}$, J. R. Catmore ${ }^{144}$, A. Cattai ${ }^{4}$, V. Cavaliere ${ }^{91}$, V. Cavasinni ${ }^{72,140}$, E. Celebi ${ }^{169}$, F. Celli ${ }^{56}$, K. Cerny ${ }^{170}$, A. S. Cerqueira ${ }^{78}$, A. Cerri ${ }^{9}$, L. Cerrito ${ }^{33,34}$, F. Cerutti ${ }^{32}$, A. Cervelli ${ }^{38,39}$, S. A. Cetin ${ }^{169}$, Z. Chadi ${ }^{133}$, D. Chakraborty ${ }^{18}$, J. Chan ${ }^{109}$, W. S. Chan ${ }^{43}$, W. Y. Chan ${ }^{147}$, J. D. Chapman ${ }^{121}$, B. Chargeishvilii17, D. G. Charlton ${ }^{49}$, T. P. Charman ${ }^{138}$, M. Chatterjee ${ }^{63}$, C. C. Chau ${ }^{131}$, S. Che ${ }^{149}$, S. Chekanovi2 , S. V. Chekulaev ${ }^{166}$, G. A. Chelkov ${ }^{30,252}$, B. Chen ${ }^{59}$, C. Chen ${ }^{116}$, C. H. Chen ${ }^{59}$, H. Chen ${ }^{172}$, H. Chen ${ }^{91}$, J. Chen ${ }^{116}$, J. Chen ${ }^{69}$, J. Chen ${ }^{134}$, S. Chen ${ }^{173}$, S. J. Chen ${ }^{172}$, X. Chen ${ }^{174}$, Y. Chen ${ }^{116}$, Y-H. Chen ${ }^{58}$, H. C. Cheng ${ }^{175}$, H. J. Cheng ${ }^{98}$, A. Cheplakov ${ }^{30}$, E. Cheremushkina ${ }^{148}$, R. Cherkaoui El Moursli120, E. Cheu ${ }^{135}$, K. Cheung ${ }^{176}$, T. J. A. Chevalérias ${ }^{61}$, L. Chevalier ${ }^{61}$, V. Chiarella ${ }^{74}$, G. Chiarelli72, G. Chiodinii ${ }^{177}$, A. S. Chisholm ${ }^{49}$, A. Chitan ${ }^{41}$, I. Chiu ${ }^{86}$, Y. H. Chiu ${ }^{40}$, M. V. Chizhov ${ }^{30}$, K. Choi ${ }^{62}$, A. R. Chomont ${ }^{75,100,}$ Y. Chou ${ }^{3}$, Y. S. Chow ${ }^{43}$, L. D. Christopher ${ }^{178}$, M. C. Chu ${ }^{175}$, X. Chu ${ }^{98,179}$, J. Chudoba ${ }^{180}$, J. J. Chwastowski ${ }^{101}$, L. Chytka ${ }^{170}$, D. Cieri' ${ }^{114}$, K. M. Ciesla ${ }^{101}$, V. Cindro ${ }^{181}$, I. A. Cioaráa1 , A. Ciocio ${ }^{32}$, F. Cirotto ${ }^{50,51}$, Z. H. Citron ${ }^{107,253}$, M. Citterio ${ }^{47}$, D. A. Ciubotaru ${ }^{41}$, B. M. Ciungu ${ }^{7}$, A. Clark ${ }^{20}$, P. J. Clark ${ }^{167}$, S. E. Clawson ${ }^{142}$, C. Clement ${ }^{64,65}$, L. Clissa ${ }^{38,39}$, Y. Coadou', M. Cobal ${ }^{13,182}$, A. Coccaro ${ }^{112}$, J. Cochran ${ }^{59}$, R. Coelho Lopes De Sa ${ }^{3}$, H. Cohen ${ }^{10}$, A. E. C. Coimbra' ${ }^{4}$, B. Cole ${ }^{69}$, A. P. Colijn ${ }^{43}$, J. Collot ${ }^{183}$, P. Conde Muiño ${ }^{27,184}$, S. H. Connell ${ }^{150}$, I. A. Connelly ${ }^{119}$, S. Constantinescu ${ }^{41}$, F. Conventi ${ }^{50,254}$, A. M. Cooper-Sarkar ${ }^{56}$, F. Cormier ${ }^{185}$, K. J. R. Cormier7 , L. D. Corpe ${ }^{113}$, M. Corradi75,100, E. E. Corrigan ${ }^{36}$, 
F. Corriveau ${ }^{31,255}$, M. J. Costa ${ }^{77}$, F. Costanza ${ }^{16}$, D. Costanzo ${ }^{60}$, G. Cowan ${ }^{6}$, J. W. Cowley ${ }^{121}$, J. Crane ${ }^{142}$, K. Cranmer ${ }^{186}$, R. A. Creager ${ }^{173}$, S. Crépé-Renaudin ${ }^{183}$, F. Crescioli ${ }^{125}$, M. Cristinziani ${ }^{123}$, V. Croft ${ }^{126}$, G. Crosetti ${ }^{163,164}$, A. Cueto ${ }^{16}$, T. Cuhadar Donszelmann ${ }^{82}$, H. Cui ${ }^{98,179}$, A. R. Cukierman ${ }^{115}$, W. R. Cunningham ${ }^{119}$, S. Czekierda ${ }^{101}$, P. Czodrowski ${ }^{4}$, M. M. Czurylo ${ }^{146}$,

M. J. Da Cunha Sargedas De Sousa ${ }^{187}$, J. V. Da Fonseca Pinto ${ }^{55}$, C. Da Via ${ }^{142}$, W. Dabrowski ${ }^{17}$, F. Dachs ${ }^{4}$, T. Dado ${ }^{188}$, S. Dahbi ${ }^{178}$, T. Dai ${ }^{57}$, C. Dallapiccola ${ }^{3}$, M. Dam ${ }^{8}$, G. D'amen $^{91}$, V. D'Amico ${ }^{141,161}$, J. Damp ${ }^{15}$, J. R. Dandoy ${ }^{173}$, M. F. Daneri ${ }^{189}$, M. Danninger ${ }^{102}$, V. Dao4 , G. Darbo ${ }^{112}$, O. Dartsi ${ }^{16}$, A. Dattagupta ${ }^{48}$, T. Daubney ${ }^{58}$, S. D'Auria ${ }^{47,66}$, C. David ${ }^{190}$, T. Davidek ${ }^{165}$, D. R. Davis ${ }^{79}$, I. Dawson ${ }^{60}$, K. De ${ }^{105}$, R. De Asmundis ${ }^{50}$, M. De Beurs ${ }^{43}$, S. De Castro ${ }^{38,39}$, N. De Groot ${ }^{25}$, P. de Jong ${ }^{43}$, H. De la Torre ${ }^{153}$, A. De Maria ${ }^{172}$, D. De Pedis ${ }^{75}$, A. De Salvo75, U. De Sanctis ${ }^{33,34}$, M. De Santis ${ }^{33,34}$, A. De Santo9, J. B. De Vivie De Regie ${ }^{23}$, D. V. Dedovich ${ }^{30}$, A. M. Deiana ${ }^{137}$, J. Del Peso ${ }^{54}$, Y. Delabat Diaz ${ }^{58}$, D. Delgove ${ }^{23}$, F. Deliot ${ }^{61}$, C. M. Delitzsch ${ }^{135}$, M. Della Pietra ${ }^{50,51}$, D. Della Volpe ${ }^{20}$, A. Dell'Acqua ${ }^{4}$, L. Dell'Asta ${ }^{33,34}$, M. Delmastro ${ }^{16}$, C. Delporte ${ }^{23}$, P. A. Delsart ${ }^{183}$, S. Demers ${ }^{103}$, M. Demichev ${ }^{30}$, G. Demontigny ${ }^{80}$, S. P. Denisov ${ }^{148}$, L. D'Eramo ${ }^{18}$, D. Derendarz ${ }^{101}$, J. E. Derkaoui ${ }^{90}$, F. Derue ${ }^{125}$,

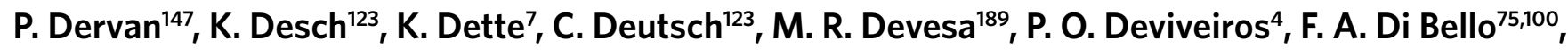
A. Di Ciaccio33,34, L. Di Ciaccio ${ }^{16}$, C. Di Donato50,51, A. Di Girolamo4 , G. Di Gregorio72,140, A. Di Luca191,192, B. Di Micco ${ }^{141,161}$, R. Di Nardo ${ }^{141,161}$, K. F. Di Petrillo ${ }^{88}$, R. Di Sipio7, C. Diaconu1', F. A. Dias ${ }^{43}$, T. Dias Do Vale ${ }^{27}$, M. A. Diaz ${ }^{193}$, F. G. Diaz Capriles ${ }^{123}$, J. Dickinson ${ }^{32}$, M. Didenko ${ }^{46}$, E. B. Diehl ${ }^{57}$, J. Dietrich ${ }^{96}$, S. Díez Cornell58, C. Diez Pardos ${ }^{122}$, A. Dimitrievska ${ }^{32}$, W. Ding ${ }^{174}$, J. Dingfelder ${ }^{123}$, S. J. Dittmeier ${ }^{146}$, F. Dittus ${ }^{4}$, F. Djama', T. Djobava ${ }^{171}$, J. I. Djuvsland ${ }^{156}$, M. A. B. Do Vale ${ }^{194}$, M. Dobre ${ }^{41}$, D. Dodsworth ${ }^{134}$, C. Doglioni ${ }^{36}$, J. Dolejsi ${ }^{165}$, Z. Dolezal ${ }^{165}$, M. Donadelli"195, B. Dong ${ }^{196}$, J. Donini ${ }^{24}$, A. D'onofrio ${ }^{172}$, M. D'Onofrio ${ }^{147}$, J. Dopke ${ }^{21}$, A. Doria ${ }^{50}$, M. T. Dova ${ }^{52}$, A. T. Doyle ${ }^{119}$, E. Drechsler ${ }^{102}$, E. Dreyer ${ }^{102}$, T. Dreyer ${ }^{5}$, A. S. Drobac ${ }^{126}$, D. Dü ${ }^{187}$, T. A. du Pree ${ }^{43}$, Y. Duan ${ }^{152}$, F. Dubinin ${ }^{37}$, M. Dubovsky ${ }^{92}$, A. Dubreuil20, E. Duchovni ${ }^{107}$, G. Duckeck ${ }^{83}$, O. A. Ducu ${ }^{4}$, D. Duda ${ }^{114}$, A. Dudarev ${ }^{4}$, A. C. Dudder ${ }^{15}$, E. M. Duffield ${ }^{32}$, M. D'uffizi'142, L. Duflot ${ }^{23}$, M. Dührssen ${ }^{4}$, C. Dülsen ${ }^{97}$, M. Dumancic ${ }^{107}$, A. E. Dumitriu ${ }^{41}$, M. Dunford ${ }^{67}$, S. Dungs ${ }^{188}$, A. Duperrin', H. Duran Yildiz ${ }^{159}$, M. Düren ${ }^{158}$, A. Durglishvili ${ }^{171}$, D. Duschinger ${ }^{136}$, B. Dutta ${ }^{58}$, D. Duvnjak ${ }^{197}$, G. I. Dyckes ${ }^{173}$, M. Dyndal ${ }^{4}$, S. Dysch $^{142}$, B. S. Dziedzic ${ }^{101}$, M. G. Eggleston ${ }^{79}$, T. Eifert ${ }^{105}$, G. Eigen ${ }^{156}$, K. Einsweiler ${ }^{32}$, T. Ekelof ${ }^{89}$, H. El Jarrari ${ }^{120}$, V. Ellajosyula ${ }^{89}$, M. Ellert ${ }^{89}$, F. Ellinghaus ${ }^{97}$, A. A. Elliot ${ }^{138}$, N. Ellis ${ }^{4}$, J. Elmsheuser ${ }^{91}$, M. Elsing ${ }^{4}$, D. Emeliyanov ${ }^{21}$, A. Emerman ${ }^{69}$, Y. Enari ${ }^{86}$, M. B. Epland ${ }^{79}$, J. Erdmann ${ }^{188}$, A. Ereditato ${ }^{63}$, P. A. Erland ${ }^{101}$, M. Errenst ${ }^{97}$, M. Escalier ${ }^{23}$, C. Escobar ${ }^{77}$, O. Estrada Pastor ${ }^{77}$, E. Etzion ${ }^{10}$, G. E. Evans ${ }^{27}$, H. Evans ${ }^{160}$, M. O. Evans ${ }^{9}$, A. Ezhilov ${ }^{117}$, F. Fabbri' ${ }^{119}$, L. Fabbri38,39, V. Fabiani ${ }^{25}$, G. Facini ${ }^{127}$, R. M. Fakhrutdinov ${ }^{148}$, S. Falciano ${ }^{75}$, P. J. Falke ${ }^{123}$, S. Falke4, J. Faltova ${ }^{165}$, Y. Fang ${ }^{98}$, Y. Fang ${ }^{98}$, G. Fanourakis ${ }^{198}$, M. Fanti ${ }^{47,66}$, M. Faraj ${ }^{13,182}$, A. Farbin ${ }^{105}$, A. Farilla ${ }^{141}$, E. M. Farina ${ }^{199,200}$, T. Farooque ${ }^{153}$, S. M. Farrington ${ }^{167}$, P. Farthouat ${ }^{4}$, F. Fassi' ${ }^{120}$, P. Fassnacht ${ }^{4}$, D. Fassouliotis ${ }^{68}$, M. Faucci Giannelli ${ }^{167}$, W. J. Fawcett ${ }^{121}$, L. Fayard ${ }^{23}$, O. L. Fedin ${ }^{117,256}$, W. Fedorko ${ }^{185}$, A. Fehr ${ }^{63}$, M. Feickert ${ }^{95}$, L. Feligioni' , A. Fell ${ }^{60}$, C. Feng ${ }^{187}$, M. Feng ${ }^{79}$, M. J. Fenton ${ }^{82}$, A. B. Fenyuk ${ }^{148}$, S. W. Ferguson ${ }^{201}$, J. Ferrando ${ }^{58}$, A. Ferrari ${ }^{89}$, P. Ferrari ${ }^{43}$, R. Ferrari' ${ }^{199}$, D. E. Ferreira de Lima ${ }^{146}$, A. Ferrer ${ }^{77}$, D. Ferrere ${ }^{20}$, C. Ferrettij ${ }^{57}$, F. Fiedler ${ }^{15}$, A. Filipčič ${ }^{181}$, F. Filthaut ${ }^{25}$, K. D. Finelli157, M. C. N. Fiolhais ${ }^{27,202,257}$, L. Fiorini ${ }^{77}$, F. Fischer ${ }^{83}$, J. Fischer ${ }^{15}$, W. C. Fisher ${ }^{153}$, T. Fitschen ${ }^{49}$, I. Fleck ${ }^{122}$, P. Fleischmann ${ }^{57}$, T. Flick ${ }^{97}$, B. M. Flierl ${ }^{83}$, L. Flores ${ }^{173}$, L. R. Flores Castillo ${ }^{175}$, F. M. Follega ${ }^{191,192}$, N. Fomin ${ }^{156}$, J. H. Foo7, G. T. Forcolin ${ }^{191,192}$, B. C. Forland ${ }^{160}$, A. Formica61, F. A. Förster ${ }^{145}$, A. C. Forti ${ }^{142}$, E. Fortin1, M. G. Foti ${ }^{56}$, D. Fournier ${ }^{23}$, H. Fox ${ }^{118}$, P. Francavilla ${ }^{72,140}$, S. Francescato ${ }^{75,100}$, M. Franchini ${ }^{38,39}$, S. Franchino ${ }^{67}$, D. Francis ${ }^{4}$, L. Franco ${ }^{16}$, L. Franconi ${ }^{63}$, M. Franklin ${ }^{88}$, 
G. Frattari ${ }^{75,100}$, A. N. Fray ${ }^{138}$, P. M. Freeman ${ }^{49}$, B. Freund ${ }^{80}$, W. S. Freund ${ }^{55}$, E. M. Freundlich ${ }^{188}$, D. C. Frizzell2 ${ }^{2}$, D. Froidevaux ${ }^{4}$, J. A. Frost ${ }^{56}$, M. Fujimoto ${ }^{85}$, C. Fukunaga ${ }^{203}$, E. Fullana Torregrosa ${ }^{77}$, T. Fusayasu ${ }^{204}$, J. Fuster ${ }^{77}$, A. Gabrielli ${ }^{38,39}$, A. Gabrielli ${ }^{4}$, S. Gadatsch ${ }^{20}$, P. Gadow ${ }^{114}$, G. Gagliardi ${ }^{111,112}$, L. G. Gagnon ${ }^{80}$, G. E. Gallardo ${ }^{56}$, E. J. Gallas ${ }^{56}$, B. J. Gallop ${ }^{21}$, R. Gamboa Goni ${ }^{138}$, K. K. Gan ${ }^{149}$, S. Ganguly ${ }^{107}$, J. Gao ${ }^{116}$, Y. Gao ${ }^{167}$, Y. S. Gao ${ }^{124,258}$, F. M. Garay Walls ${ }^{193}$, C. García77, J. E. García Navarro77, J. A. García Pascual98, C. Garcia-Argos ${ }^{81}$, M. Garcia-Sciveres ${ }^{32}$, R. W. Gardner ${ }^{205}$, N. Garelli115, S. Gargiulo ${ }^{81}$, C. A. Garner7, V. Garonne ${ }^{144}$, S. J. Gasiorowski ${ }^{53}$, P. Gaspar ${ }^{55}$, A. Gaudiello ${ }^{111,112}$, G. Gaudio ${ }^{199}$, P. Gauzzi ${ }^{75,100}$, I. L. Gavrilenko ${ }^{37}$, A. Gavrilyuk ${ }^{206}$, C. Gay ${ }^{185}$, G. Gaycken ${ }^{58}$, E. N. Gazis ${ }^{42}$, A. A. Geanta ${ }^{41}$, C. M. Gee ${ }^{22}$, C. N. P. Gee ${ }^{21}$, J. Geisen ${ }^{36}$, M. Geisen ${ }^{15}$, C. Gemme ${ }^{112}$, M. H. Genest ${ }^{183}$, C. Geng ${ }^{57}$, S. Gentile ${ }^{75,100}$, S. George ${ }^{6}$, T. Geralis ${ }^{198}$, L. O. Gerlach ${ }^{5}$, P. Gessinger-Befurt ${ }^{15}$, G. Gessner ${ }^{188}$, M. Ghasemi Bostanabad ${ }^{40}$, M. Ghneimat ${ }^{122}$, A. Ghosh ${ }^{23}$, A. Ghosh ${ }^{162}$, B. Giacobbe ${ }^{39}$, S. Giagu ${ }^{75,100,}$ N. Giangiacomi ${ }^{7}$, P. Giannetti ${ }^{72}$, A. Giannini ${ }^{50,51}$, G. Giannini ${ }^{145}$, S. M. Gibson ${ }^{6}$, M. Gignac $^{22}$, D. T. Gil ${ }^{207}$, B. J. Gilbert ${ }^{69}$, D. Gillberg ${ }^{131}$, G. Gilles ${ }^{97}$, N. E. K. Gillwald ${ }^{58}$, D. M. Gingrich ${ }^{143,247}$, M. P. Giordani ${ }^{13,182}$, P. F. Giraud ${ }^{61}$, G. Giugliarelli13,182, D. Giugni ${ }^{47}$, F. Giuli33,34, S. Gkaitatzis ${ }^{99}$, I. Gkialas ${ }^{68,259}$,

E. L. Gkougkousis ${ }^{145}$, P. Gkountoumis ${ }^{42}$, L. K. Gladilin ${ }^{208}$, C. Glasman ${ }^{54}$, J. Glatzer ${ }^{145}$, P. C. F. Glaysher ${ }^{58}$, A. Glazov ${ }^{58}$, G. R. Gledhill ${ }^{48}$, I. Gnesi ${ }^{164,260}$, M. Goblirsch-Kolb ${ }^{134}$, D. Godin ${ }^{80}$, S. Goldfarb ${ }^{110}$, T. Golling ${ }^{20}$, D. Golubkov ${ }^{148}$, A. Gomes ${ }^{27,209}$, R. Goncalves Gama5 , R. Gonçalo ${ }^{27,202}$, G. Gonella48, L. Gonella ${ }^{49}$, A. Gongadze ${ }^{30}$, F. Gonnella ${ }^{49}$, J. L. Gonski ${ }^{69}$, S. González de la Hoz ${ }^{77}$, S. Gonzalez Fernandez ${ }^{145}$, R. Gonzalez Lopez ${ }^{147}$, C. Gonzalez Renteria ${ }^{32}$, R. Gonzalez Suarez ${ }^{89}$, S. Gonzalez-Sevilla ${ }^{20}$, G. R. Gonzalvo Rodriguez ${ }^{77}$, L. Goossens ${ }^{4}$, N. A. Gorasia ${ }^{49}$, P. A. Gorbounov ${ }^{206}$, H. A. Gordon ${ }^{91}$, B. Gorini ${ }^{4}$, E. Gorini ${ }^{177,210}$, A. Gorišek ${ }^{181}$, A. T. Goshaw ${ }^{79}$, M. I. Gostkin ${ }^{30}$, C. A. Gottardo ${ }^{25}$, M. Gouighri211,212, A. G. Goussiou ${ }^{53}$, N. Govender ${ }^{150}$, C. Goy ${ }^{16}$, I. Grabowska-Bold17, E. C. Graham ${ }^{147}$, J. Gramling ${ }^{82}$, E. Gramstad ${ }^{144}$, S. Grancagnolo96, M. Grandi ${ }^{9}$, V. Gratchev ${ }^{117}$, P. M. Gravila ${ }^{213}$, F. G. Gravili177,210, C. Gray ${ }^{119}$, H. M. Gray ${ }^{32}$, C. Grefe ${ }^{123}$, K. Gregersen ${ }^{36}$, I. M. Gregor ${ }^{58}$, P. Grenier ${ }^{115}$, K. Grevtsov ${ }^{58}$, C. Grieco ${ }^{145}$, N. A. Grieser ${ }^{2}$, A. A. Grillo22, K. Grimm ${ }^{124,261}$, S. Grinstein ${ }^{145,262}$, J.-F. Grivaz ${ }^{23}$, S. Groh ${ }^{15}$, E. Gross ${ }^{107}$, J. Grosse-Knetter ${ }^{5}$, Z. J. Grout ${ }^{113}$, C. Grud ${ }^{57}$, A. Grummer ${ }^{214}$, J. C. Grundy ${ }^{56}$, L. Guan ${ }^{57}$, W. Guan ${ }^{109}$, C. Gubbels ${ }^{185}$, J. Guenther ${ }^{215}$, A. Guerguichon ${ }^{23}$, J. G. R. Guerrero Rojas ${ }^{77}$, F. Guescini" ${ }^{114}$, D. Guest ${ }^{215}$, R. Gugel ${ }^{15}$, A. Guida ${ }^{58}$, T. Guillemin ${ }^{16}$, S. Guindon 4, J. Guo ${ }^{196}$, W. Guo ${ }^{57}$, Y. Guo ${ }^{116}$, Z. Guo ${ }^{1}$, R. Gupta ${ }^{58}$, S. Gurbuz ${ }^{19}$, G. Gustavino², M. Guth ${ }^{81}$, P. Gutierrez ${ }^{2}$, C. Gutschow ${ }^{113}$, C. Guyot ${ }^{61}$, C. Gwenlan ${ }^{56}$, C. B. Gwilliam ${ }^{147}$, E. S. Haaland ${ }^{144}$, A. Haas ${ }^{186}$, C. Haber ${ }^{32}$, H. K. Hadavand ${ }^{105}$, A. Hadef ${ }^{15}$, M. Haleem ${ }^{139}$, J. Haley ${ }^{73}$, J. J. Hall ${ }^{60}$, G. Halladjian ${ }^{153}$, G. D. Hallewell' ${ }^{1}$ K. Hamano ${ }^{40}$, H. Hamdaoui ${ }^{120}$, M. Hamer ${ }^{123}$, G. N. Hamity ${ }^{167}$, K. Han ${ }^{116}$, L. Han ${ }^{172}$, L. Han ${ }^{116}$, S. Han ${ }^{32}$, Y. F. Han', K. Hanagaki ${ }^{76,263}$, M. Hance ${ }^{22}$, D. M. Handl83, M. D. Hank ${ }^{205}$, R. Hankache ${ }^{125}$, E. Hansen ${ }^{36}$, J. B. Hansen ${ }^{8}$, J. D. Hansen ${ }^{8}$, M. C. Hansen ${ }^{123}$, P. H. Hansen ${ }^{8}$, E. C. Hanson ${ }^{142}$, K. Hara ${ }^{216}$, T. Harenberg 97 ,

S. Harkusha ${ }^{217}$, P. F. Harrison ${ }^{127}$, N. M. Hartman ${ }^{115}$, N. M. Hartmann ${ }^{83}$, Y. Hasegawa ${ }^{218}$, A. Hasib ${ }^{167}$, S. Hassani ${ }^{61}$, S. Haug ${ }^{63}$, R. Hauser ${ }^{153}$, M. Havranek ${ }^{44}$, C. M. Hawkes ${ }^{49}$, R. J. Hawkings ${ }^{4}$, S. Hayashida ${ }^{84}$, D. Hayden ${ }^{153}$, C. Hayes ${ }^{57}$, R. L. Hayes ${ }^{185}$, C. P. Hays ${ }^{56}$, J. M. Hays ${ }^{138}$, H. S. Hayward ${ }^{147}$, S. J. Haywood ${ }^{21}$, F. He ${ }^{116}$, Y. He ${ }^{87}$, M. P. Heath ${ }^{167}$, V. Hedberg ${ }^{36}$, A. L. Heggelund ${ }^{144}$, N. D. Hehir ${ }^{138}$, C. Heidegger ${ }^{81}$, K. K. Heidegger ${ }^{81}$, W. D. Heidorn ${ }^{59}$, J. Heilman ${ }^{131}$, S. Heim ${ }^{58}$, T. Heim ${ }^{32}$, B. Heinemann ${ }^{58,264}$, J. G. Heinlein ${ }^{173}$, J. J. Heinrich ${ }^{48}$, L. Heinrich4 ${ }^{4}$ J. Hejbal ${ }^{180}$, L. Helary ${ }^{58}$, A. Held ${ }^{186}$, S. Hellesund ${ }^{144}$, C. M. Helling ${ }^{22}$, S. Hellman ${ }^{64,65}$, C. Helsens ${ }^{4}$, R. C. W. Henderson ${ }^{118}$, L. Henkelmann ${ }^{121}$, A. M. Henriques Correia ${ }^{4}$, H. Herde ${ }^{134}$, Y. Hernández Jiménez ${ }^{178}$, H. Herr ${ }^{15}$, M. G. Herrmann ${ }^{83}$, T. Herrmann ${ }^{136}$, G. Herten ${ }^{81}$, R. Hertenberger ${ }^{83}$, L. Hervas ${ }^{4}$, G. G. Hesketh ${ }^{113}$, N. P. Hessey ${ }^{166}$, H. Hibi219, 
S. Higashino ${ }^{76}$, E. Higón-Rodriguez ${ }^{77}$, K. Hildebrand ${ }^{205}$, J. C. Hill'121, K. K. Hill'1 , K. H. Hiller ${ }^{58}$, S. J. Hillier ${ }^{49}$, M. Hils ${ }^{136}$, I. Hinchliffe ${ }^{32}$, F. Hinterkeuser ${ }^{123}$, M. Hirose ${ }^{220}$, S. Hirose ${ }^{216}$, D. Hirschbuehl ${ }^{97}$, B. Hiti' ${ }^{181}$, O. Hladik ${ }^{180}$, J. Hobbs ${ }^{130}$, R. Hobincu ${ }^{221}$, N. Hod ${ }^{107}$, M. C. Hodgkinson ${ }^{60}$, A. Hoecker ${ }^{4}$, D. Hohn ${ }^{81}$, D. Hohov ${ }^{23}$, T. Holm ${ }^{123}$, T. R. Holmes ${ }^{205}$, M. Holzbock ${ }^{114}$, L. B. A. H. Hommels ${ }^{121}$,

T. M. Hong ${ }^{108}$, J. C. Honig ${ }^{81}$, A. Hönle ${ }^{114}$, B. H. Hooberman ${ }^{95}$, W. H. Hopkins ${ }^{12}$, Y. Horii ${ }^{84}$, P. Horn ${ }^{136}$, L. A. Horyn ${ }^{205}$, S. Hou ${ }^{45}$, A. Hoummada ${ }^{133}$, J. Howarth ${ }^{119}$, J. Hoya ${ }^{52}$, M. Hrabovsky ${ }^{170}$, J. Hrivnac ${ }^{23}$, A. Hrynevich ${ }^{222}$, T. Hryn'ova ${ }^{16}$, P. J. Hsu'76, S.-C. Hsu ${ }^{53}$, Q. Hu ${ }^{69}$, S. Hu ${ }^{196}$, Y. F. Hu ${ }^{98,179,265}$, D. P. Huang ${ }^{113}$, X. Huang ${ }^{172}$, Y. Huang ${ }^{116}$, Y. Huang ${ }^{98}$, Z. Hubacek ${ }^{44}$, F. Hubaut ${ }^{1}$, M. Huebner ${ }^{123}$, F. Huegging ${ }^{123}$, T. B. Huffman ${ }^{56}$, M. Huhtinen ${ }^{4}$, R. Hulsken ${ }^{183}$, R. F. H. Hunter ${ }^{131}$, N. Huseynov ${ }^{30,266}$, J. Huston ${ }^{153}$, J. Huth ${ }^{88}$, R. Hyneman ${ }^{115}$, S. Hyrych ${ }^{92}$, G. lacobucci $^{20}$, G. lakovidis ${ }^{91}$, I. Ibragimov ${ }^{122}$, L. Iconomidou-Fayard ${ }^{23}$, P. lengo ${ }^{4}$, R. Ignazzi ${ }^{8}$, R. Iguchi ${ }^{86}$, T. lizawa ${ }^{20}$, Y. Ikegami ${ }^{76}$, M. Ikeno ${ }^{76}$, N. Ilic ${ }^{7,25,255}$, F. Iltzsche ${ }^{136}$, H. Imam ${ }^{133}$, G. Introzzi ${ }^{199,200}$, M. Iodice ${ }^{141}$, K. Iordanidou ${ }^{166}$, V. Ippolito ${ }^{75,100}$, M. F. Isacson ${ }^{89}$, M. Ishino ${ }^{86}$, W. Islam ${ }^{73}$, C. Issever ${ }^{58,96}$, S. Istin ${ }^{11}$, J. M. Iturbe Ponce ${ }^{175}$, R. Iuppa ${ }^{191,192}$, A. Ivina ${ }^{107}$, J. M. Izen ${ }^{201}$, V. Izzo ${ }^{50}$, P. Jacka ${ }^{180}$, P. Jackson ${ }^{197}$, R. M. Jacobs ${ }^{58}$, B. P. Jaeger ${ }^{102}$, V. Jain ${ }^{223}$, G. Jäkel ${ }^{97}$, K. B. Jakobi ${ }^{15}$, K. Jakobs ${ }^{81}$, T. Jakoubek ${ }^{107}$, J. Jamieson ${ }^{119}$, K. W. Janas ${ }^{17}$, R. Jansky ${ }^{20}$, M. Janus ${ }^{5}$, P. A. Janus ${ }^{17}$, G. Jarlskog ${ }^{36}$, A. E. Jaspan ${ }^{147}$, N. Javadov ${ }^{30,266}$, T. Javůrek ${ }^{4}$, M. Javurkova ${ }^{3}$, F. Jeanneau ${ }^{61}$, L. Jeanty ${ }^{48}$, J. Jejelava ${ }^{224}$, P. Jenni ${ }^{81,267}$, N. Jeong ${ }^{58}$, S. Jézéquel ${ }^{16}$, J. Jia ${ }^{130}$, Z. Jia ${ }^{172}$, H. Jiang ${ }^{59}$, Y. Jiang ${ }^{116}$, Z. Jiang ${ }^{115}$, S. Jiggins ${ }^{81}$, F. A. Jimenez Morales ${ }^{24}$, J. Jimenez Pena ${ }^{114}$, S. Jin ${ }^{172}$, A. Jinaru ${ }^{41}$, O. Jinnouchi ${ }^{87}$, H. Jivan ${ }^{178}$, P. Johansson ${ }^{60}$, K. A. Johns ${ }^{135}$, C. A. Johnson ${ }^{160}$, E. Jones ${ }^{127}$, R. W. L. Jones ${ }^{118}$, S. D. Jones ${ }^{9}$, T. J. Jones ${ }^{147}$, J. Jovicevic ${ }^{4}$, X. Ju ${ }^{32}$, J. J. Junggeburth ${ }^{114}$, A. Juste Rozas ${ }^{145,262}$, A. Kaczmarska ${ }^{101}$, M. Kado ${ }^{75,100}$, H. Kagan ${ }^{149}$, M. Kagan ${ }^{115}$, A. Kahn ${ }^{69}$, C. Kahra ${ }^{15}$, T. Kaji225, E. Kajomovitz ${ }^{11}$, C. W. Kalderon ${ }^{91}$, A. Kaluza ${ }^{15}$, A. Kamenshchikov ${ }^{148}$, M. Kaneda ${ }^{86}$, N. J. Kang ${ }^{22}$, S. Kang ${ }^{59}$, Y. Kano ${ }^{84}$, J. Kanzaki ${ }^{76}$, L. S. Kaplan ${ }^{109}$, D. Kar ${ }^{178}$, K. Karava ${ }^{56}$, M. J. Kareem ${ }^{190}$, I. Karkanias ${ }^{99}$, S. N. Karpov ${ }^{30}$, Z. M. Karpova ${ }^{30}$, V. Kartvelishvili"18, A. N. Karyukhin ${ }^{148}$, E. Kasimi ${ }^{99}$, A. Kastanas ${ }^{64,65}$, C. Kato ${ }^{152}$, J. Katzy ${ }^{58}$, K. Kawade ${ }^{218}$, K. Kawagoe ${ }^{226}$, T. Kawaguchi ${ }^{84}$, T. Kawamoto ${ }^{61}$, G. Kawamura ${ }^{5}$, E. F. Kay ${ }^{40}$, F. I. Kaya ${ }^{126}$, S. Kazakos ${ }^{145}$, V. F. Kazanin ${ }^{70,71}$, J. M. Keaveney ${ }^{93}$, R. Keeler ${ }^{40}$, J. S. Keller ${ }^{131}$, E. Kellermann ${ }^{36}$, D. Kelsey ${ }^{9}$, J. J. Kempster ${ }^{49}$, J. Kendrick ${ }^{49}$, K. E. Kennedy ${ }^{69}$, O. Kepka ${ }^{180}$, S. Kersten ${ }^{97}$, B. P. Kerševan ${ }^{181}$, S. Ketabchi Haghighat ${ }^{7}$, F. Khalil-Zada ${ }^{227}$, M. Khandoga ${ }^{61}$, A. Khanov ${ }^{73}$, A. G. Kharlamov ${ }^{70,71}$, T. Kharlamova70,71, E. E. Khoda ${ }^{185}$, T. J. Khoo ${ }^{215}$, G. Khoriauli' ${ }^{139}$, E. Khramov ${ }^{30}$, J. Khubua ${ }^{171}$, S. Kido ${ }^{219}$, M. Kiehn ${ }^{4}$, E. Kim ${ }^{87}$, Y. K. Kim ${ }^{205}$, N. Kimuraa ${ }^{113}$, A. Kirchhoff5 ${ }^{5}$ D. Kirchmeier ${ }^{136}$, J. Kirk" ${ }^{21}$, A. E. Kiryunin ${ }^{114}$, T. Kishimoto ${ }^{86}$, D. P. Kisliuk7, V. Kitali ${ }^{58}$, C. Kitsaki ${ }^{42}$, O. Kivernyk ${ }^{123}$, T. Klapdor-Kleingrothaus ${ }^{81}$, M. Klassen ${ }^{67}$, C. Klein ${ }^{131}$, M. H. Klein ${ }^{57}$, M. Klein ${ }^{147}$, U. Klein ${ }^{147}$, K. Kleinknecht ${ }^{15}$, P. Klimek ${ }^{4}$, A. Klimentov'1, F. Klimpel ${ }^{4}$, T. Klingl'123, T. Klioutchnikova4, F. F. Klitzner ${ }^{83}$, P. Kluit ${ }^{43}$, S. Kluth ${ }^{114}$, E. Kneringer ${ }^{215}$, E. B. F. G. Knoops ${ }^{1}$, A. Knue ${ }^{81}$, D. Kobayashi²26, M. Kobel ${ }^{136}$, M. Kocian ${ }^{115}$, T. Kodama ${ }^{86}$, P. Kodys ${ }^{165}$, D. M. Koeck9 , P. T. Koenig ${ }^{123}$, T. Koffas ${ }^{131}$, N. M. Köhler ${ }^{4}$, M. Kolb ${ }^{61}$, I. Koletsou ${ }^{16}$, T. Komarek ${ }^{170}$, T. Kondo ${ }^{76}$, K. Köneke ${ }^{81}$, A. X. Y. Kong ${ }^{197}$, A. C. König ${ }^{25}$, T. Kono ${ }^{85}$, V. Konstantinides ${ }^{113}$, N. Konstantinidis ${ }^{113}$, B. Konya ${ }^{36}$, R. Kopeliansky ${ }^{160}$, S. Koperny ${ }^{17}$, K. Korcyl ${ }^{101}$, K. Kordas ${ }^{99}$, G. Koren ${ }^{10}$, A. Korn ${ }^{113}$, I. Korolkov ${ }^{145}$, E. V. Korolkova ${ }^{60}$, N. Korotkova ${ }^{208}$, O. Kortner ${ }^{114}$, S. Kortner ${ }^{114}$, V. V. Kostyukhin ${ }^{46,60}$, A. Kotsokechagia ${ }^{23}$, A. Kotwal79, A. Koulouris ${ }^{42}$,

A. Kourkoumeli-Charalampidi ${ }^{199,200}$, C. Kourkoumelis ${ }^{68}$, E. Kourlitis ${ }^{12}$, V. Kouskoura ${ }^{91}$, R. Kowalewski ${ }^{40}$, W. Kozanecki ${ }^{142}$, A. S. Kozhin ${ }^{148}$, V. A. Kramarenko ${ }^{208}$, G. Kramberger ${ }^{181}$, D. Krasnopevtsev ${ }^{116}$, M. W. Krasny ${ }^{125}$, A. Krasznahorkay ${ }^{4}$, D. Krauss ${ }^{114}$, J. A. Kremer ${ }^{15}$, J. Kretzschmar ${ }^{147}$, K. Kreul ${ }^{96}$, P. Krieger ${ }^{7}$, F. Krieter ${ }^{83}$, S. Krishnamurthy ${ }^{3}$, A. Krishnan ${ }^{146}$, M. Krivos ${ }^{165}$, K. Krizka ${ }^{32}$, K. Kroeninger ${ }^{188}$, 
H. Kroha ${ }^{114}$, J. Kroll ${ }^{180}$, J. Kroll' ${ }^{173}$, K. S. Krowpman ${ }^{153}$, U. Kruchonak ${ }^{30}$, H. Krüger ${ }^{123}$, N. Krumnack ${ }^{59}$, M. C. Kruse ${ }^{79}$, J. A. Krzysiak ${ }^{101}$, A. Kubota ${ }^{87}$, O. Kuchinskaia ${ }^{46}$, S. Kuday ${ }^{228}$, D. Kuechler ${ }^{58}$, J. T. Kuechler ${ }^{58}$, S. Kuehn ${ }^{4}$, T. Kuhl ${ }^{58}$, V. Kukhtin ${ }^{30}$, Y. Kulchitsky ${ }^{217,268}$, S. Kuleshov ${ }^{229}$, Y. P. Kulinich ${ }^{95}$, M. Kuna ${ }^{183}$, A. Kupco ${ }^{180}$, T. Kupfer ${ }^{188}$, O. Kuprash ${ }^{81}$, H. Kurashige ${ }^{219}$, L. L. Kurchaninov ${ }^{166}$, Y. A. Kurochkin ${ }^{217}$, A. Kurova ${ }^{132}$, M. G. Kurth ${ }^{98,179}$, E. S. Kuwertz ${ }^{4}$, M. Kuze ${ }^{87}$, A. K. Kvam³, J. Kvita170, T. Kwan ${ }^{31}$, C. Lacasta ${ }^{77}$, F. Lacava ${ }^{75,100}$, D. P. J. Lack ${ }^{142}$, H. Lacker ${ }^{96}$, D. Lacour ${ }^{125}$, E. Ladygin ${ }^{30}$, R. Lafaye ${ }^{16}$, B. Laforge ${ }^{125}$, T. Lagouri230, S. Lai ${ }^{5}$, I. K. Lakomiec ${ }^{17}$, J. E. Lambert ${ }^{2}$, S. Lammers ${ }^{160}$, W. Lampl ${ }^{135}$, C. Lampoudis ${ }^{99}$, E. Lançon ${ }^{91}$, U. Landgraf ${ }^{81}$, M. P. J. Landon ${ }^{138}$, V. S. Lang ${ }^{81}$, J. C. Lange ${ }^{5}$, R. J. Langenberg ${ }^{3}$, A. J. Lankford ${ }^{82}$, F. Lanni ${ }^{91}$, K. Lantzsch ${ }^{123}$, A. Lanza199, A. Lapertosa111,12, J. F. Laporte ${ }^{61}$, T. Lari" ${ }^{47}$, F. Lasagni Manghi ${ }^{38,39}$, M. Lassnig ${ }^{4}$, V. Latonova ${ }^{180}$, T. S. Lau ${ }^{175}$, A. Laudrain ${ }^{15}$, A. Laurier ${ }^{131}$, M. Lavorgna ${ }^{50,51}$, S. D. Lawlor ${ }^{6}$, M. Lazzaroni $^{47,66}$, B. Le ${ }^{142}$, E. Le Guirriec ${ }^{1}$, A. Lebedev ${ }^{59}$, M. LeBlanc ${ }^{135}$, T. LeCompte ${ }^{12}$, F. Ledroit-Guillon ${ }^{183}$, A. C. A. Lee ${ }^{113}$, C. A. Lee ${ }^{91}$, G. R. Lee ${ }^{156}$, L. Lee ${ }^{88}$, S. C. Lee $^{45}$, S. Lee ${ }^{59}$, B. Lefebvre ${ }^{166}$, H. P. Lefebvre ${ }^{6}$, M. Lefebvre ${ }^{40}$, C. Leggett $^{32}$, K. Lehmann $^{102}$, N. Lehmann ${ }^{63}$, G. Lehmann Miotto ${ }^{4}$, W. A. Leight ${ }^{58}$, A. Leisos ${ }^{99,269}$, M. A. L. Leite ${ }^{195}$, C. E. Leitgeb ${ }^{83}$, R. Leitner ${ }^{165}$, K. J. C. Leney ${ }^{137}$, T. Lenz ${ }^{123}$, S. Leone ${ }^{72}$, C. Leonidopoulos ${ }^{167}$, A. Leopold ${ }^{125}$, C. Leroy ${ }^{80}$, R. Les ${ }^{153}$, C. G. Lester ${ }^{121}$, M. Levchenko ${ }^{117}$, J. Levêque ${ }^{16}$, D. Levin ${ }^{57}$, L. J. Levinson ${ }^{107}$, D. J. Lewis ${ }^{49}$, B. Li $^{174}$,

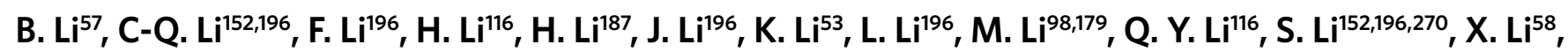

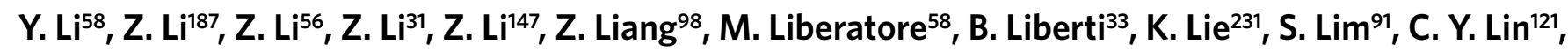
K. Lin ${ }^{153}$, R. A. Linck ${ }^{160}$, R. E. Lindley ${ }^{135}$, J. H. Lindon ${ }^{49}$, A. Linss ${ }^{58}$, A. L. Lionti ${ }^{20}$, E. Lipeles ${ }^{173}$, A. Lipniacka156, T. M. Liss ${ }^{95,271}$, A. Lister ${ }^{185}$, J. D. Little ${ }^{105}$, B. Liu ${ }^{59}$, B. X. Liu102, H. B. Liu ${ }^{91}$, J. B. Liu ${ }^{116}$,

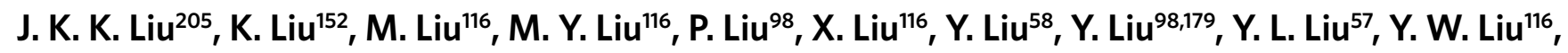
M. Livan ${ }^{199,200}$, A. Lleres ${ }^{183}$, J. Llorente Merino ${ }^{102}$, S. L. Lloyd ${ }^{138}$, C. Y. Lo ${ }^{232}$, E. M. Lobodzinska ${ }^{58}$, P. Loch ${ }^{135}$, S. Loffredo ${ }^{33,34}$, T. Lohse ${ }^{96}$, K. Lohwasser ${ }^{60}$, M. Lokajicek ${ }^{180}$, J. D. Long ${ }^{95}$, R. E. Long ${ }^{118}$, I. Longarini ${ }^{75,100}$, L. Longo ${ }^{4}$, I. Lopez Paz ${ }^{142}$, A. Lopez Solis ${ }^{60}$, J. Lorenz ${ }^{83}$, N. Lorenzo Martinez ${ }^{16}$, A. M. Lory ${ }^{83}$, A. Lösle ${ }^{81}$, X. Lou ${ }^{64,65}$, X. Lou ${ }^{98}$, A. Lounis ${ }^{23}$, J. Love ${ }^{12}$, P. A. Love ${ }^{118}$, J. J. Lozano Bahilo77, M. Lu ${ }^{116}$, Y. J. Lu ${ }^{176}$, H. J. Lubatti ${ }^{53}$, C. Luci ${ }^{75,100}$, F. L. Lucio Alves ${ }^{172}$, A. Lucotte ${ }^{183}$, F. Luehring ${ }^{160}$, I. Luise ${ }^{130}$, L. Luminari ${ }^{75}$, B. Lund-Jensen ${ }^{233}$, N. A. Luongo ${ }^{48}$, M. S. Lutz ${ }^{10}$, D. Lynn ${ }^{91}$, H. Lyons ${ }^{147}$, R. Lysak ${ }^{180}$, E. Lytken ${ }^{36}$, F. Lyu ${ }^{98}$, V. Lyubushkin ${ }^{30}$, T. Lyubushkina ${ }^{30}$, H. Ma91, L. L. Ma ${ }^{187}$, Y. Ma ${ }^{113}$, D. M. Mac Donell ${ }^{40}$, G. Maccarrone ${ }^{74}$, C. M. Macdonald 60 , J. C. MacDonald ${ }^{60}$, J. Machado Miguens ${ }^{173}$, R. Madar ${ }^{24}$, W. F. Mader ${ }^{136}$, M. Madugoda Ralalage Donn ${ }^{73}$ N. Madysa ${ }^{136}$, J. Maeda ${ }^{219}$, T. Maeno ${ }^{91}$, M. Maerker ${ }^{136}$, V. Magerl ${ }^{81}$, N. Magini ${ }^{59}$, J. Magro ${ }^{13,182,272}$, D. J. Mahon ${ }^{69}$, C. Maidantchik ${ }^{55}$, A. Maio ${ }^{27,209,234}$, K. Maj"7 , O. Majersky ${ }^{92}$, S. Majewski ${ }^{48}$, Y. Makida76, N. Makovec ${ }^{23}$, B. Malaescu ${ }^{125}$, Pa. Malecki ${ }^{101}$, V. P. Maleev ${ }^{117}$, F. Malek ${ }^{183}$, D. Malito ${ }^{163,164}$, U. Mallik ${ }^{162}$, C. Malone ${ }^{121}$, S. Maltezos ${ }^{42}$, S. Malyukov ${ }^{30}$, J. Mamuzic ${ }^{77}$, G. Mancini74, J. P. Mandalia138, I. Mandićc181,

L. Manhaes de Andrade Filho ${ }^{78}$, I. M. Maniatis ${ }^{99}$, J. Manjarres Ramos ${ }^{136}$, K. H. Mankinen ${ }^{36}$, A. Mann ${ }^{83}$, A. Manousos ${ }^{215}$, B. Mansoulie ${ }^{61}$, I. Manthos ${ }^{99}$, S. Manzoni ${ }^{43}$, A. Marantis ${ }^{99}$, G. Marceca ${ }^{189}$, L. Marchese ${ }^{56}$, G. Marchiori' ${ }^{125}$, M. Marcisovsky ${ }^{180}$, L. Marcoccia ${ }^{33,34}$, C. Marcon ${ }^{36}$, M. Marjanovic ${ }^{2}$, Z. Marshall32, M. U. F. Martensson ${ }^{89}$, S. Marti-Garcia77, C. B. Martin ${ }^{149}$, T. A. Martin'127, V. J. Martin ${ }^{167}$, B. Martin dit Latour ${ }^{156}$, L. Martinelli' ${ }^{141,161}$, M. Martinez ${ }^{145,262}$, P. Martinez Agullo77, V. I. Martinez Outschoorn ${ }^{3}$, S. Martin-Haugh ${ }^{21}$, V. S. Martoiu ${ }^{41}$, A. C. Martyniuk ${ }^{113}$, A. Marzin ${ }^{4}$, S. R. Maschek ${ }^{114}$, L. Masetti ${ }^{15}$, T. Mashimo ${ }^{86}$, R. Mashinistov ${ }^{37}$, J. Masik ${ }^{142}$, A. L. Maslennikov ${ }^{70,71}$, L. Massa ${ }^{38,39}$, P. Massarotti ${ }^{50,51}$, P. Mastrandrea ${ }^{72,140}$, A. Mastroberardino ${ }^{163,164}$, T. Masubuchi ${ }^{86}$, D. Matakias ${ }^{91}$, A. Matic ${ }^{83}$, N. Matsuzawa ${ }^{86}$, P. Mättig ${ }^{123}$, J. Maurer ${ }^{41}$, B. Maček ${ }^{181}$, D. A. Maximov ${ }^{70,71}$, 
R. Mazini ${ }^{45}$, I. Maznas ${ }^{99}$, S. M. Mazza22, J. P. Mc Gowan ${ }^{31}$, S. P. Mc Kee ${ }^{57}$, T. G. McCarthy ${ }^{114}$, W. P. McCormack ${ }^{32}$, E. F. McDonald ${ }^{110}$, A. E. McDougall ${ }^{43}$, J. A. Mcfayden ${ }^{32}$, G. Mchedlidze ${ }^{171}$, M. A. McKay ${ }^{137}$, K. D. McLean40, S. J. McMahon'21, P. C. McNamara'110, C. J. McNicol'127, R. A. McPherson ${ }^{40,255}$, J. E. Mdhluli178, Z. A. Meadows ${ }^{3}$, S. Meehan ${ }^{4}$, T. Megy ${ }^{24}$, S. Mehlhase ${ }^{83}$, A. Mehta ${ }^{147}$, B. Meirose ${ }^{201}$, D. Melini ${ }^{11}$, B. R. Mellado Garcia'17, J. D. Mellenthin ${ }^{5}$, M. Melo ${ }^{92}$, F. Meloni ${ }^{58}$, A. Melzer ${ }^{123}$, E. D. Mendes Gouveia ${ }^{27,168}$, A. M. Mendes Jacques Da Costa ${ }^{49}$, H. Y. Meng ${ }^{7}$, L. Meng ${ }^{4}$, X. T. Meng ${ }^{57}$, S. Menke ${ }^{114}$, E. Meoni ${ }^{163,164}$, S. Mergelmeyer ${ }^{96}$, S. A. M. Merkt ${ }^{108}$, C. Merlassino ${ }^{56}$, P. Mermod ${ }^{20}$, L. Merola ${ }^{50,51}$, C. Meroni ${ }^{47}$, G. Merz ${ }^{57}$, O. Meshkov37,208, J. K. R. Meshreki'122, J. Metcalfe ${ }^{12}$, A. S. Mete ${ }^{12}$, C. Meyer ${ }^{160}$, J-P. Meyer ${ }^{61}$, M. Michetti ${ }^{96}$, R. P. Middleton ${ }^{21}$, L. Mijović ${ }^{167}$, G. Mikenberg ${ }^{107}$, M. Mikestikova ${ }^{180}$, M. Mikuž ${ }^{181}$, H. Mildner ${ }^{60}$, A. Milic ${ }^{7}$, C. D. Milke ${ }^{137}$, D. W. Miller ${ }^{205}$, L. S. Miller ${ }^{131}$, A. Milov ${ }^{107}$, D. A. Milstead ${ }^{64,65}$, A. A. Minaenko ${ }^{148}$, I. A. Minashvili ${ }^{171}$, L. Mince ${ }^{119}$, A. I. Mincer ${ }^{186}$, B. Mindur ${ }^{17}$, M. Mineev ${ }^{30}$, Y. Minegishi ${ }^{86}$, Y. Mino ${ }^{35}$, L. M. Mir ${ }^{145}$, M. Mironova ${ }^{56}$, T. Mitani ${ }^{225}$, J. Mitrevski ${ }^{83}$, V. A. Mitsou ${ }^{77}$, M. Mittal196, O. Miu ${ }^{7}$, A. Miucci ${ }^{63}$, P. S. Miyagawa ${ }^{138}$, A. Mizukami ${ }^{76}$,

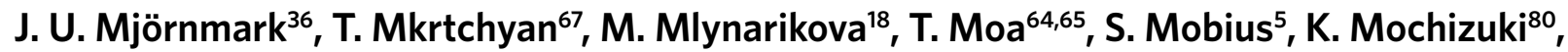
P. Moder ${ }^{58}$, P. Mogg ${ }^{83}$, S. Mohapatra ${ }^{69}$, R. Moles-Valls ${ }^{123}$, K. Mönig'58, E. Monnier ${ }^{1}$, A. Montalbano ${ }^{102}$, J. Montejo Berlingen ${ }^{4}$, M. Montella ${ }^{113}$, F. Monticelli52 , S. Monzani ${ }^{47}$, N. Morange ${ }^{23}$, A. L. Moreira De Carvalho ${ }^{27}$, D. Moreno ${ }^{235}$, M. Moreno Llácer77, C. Moreno Martinez ${ }^{145}$, P. Morettini ${ }^{112}$, M. Morgenstern ${ }^{11}$, S. Morgenstern ${ }^{136}$, D. Mori' ${ }^{102}$, M. Morii ${ }^{88}$, M. Morinaga ${ }^{225}$, V. Morisbak ${ }^{144}$, A. K. Morley4, G. Mornacchi ${ }^{4}$, A. P. Morris ${ }^{113}$, L. Morvaj ${ }^{4}$, P. Moschovakos ${ }^{4}$, B. Moser ${ }^{43}$, M. Mosidze ${ }^{171}$, T. Moskalets ${ }^{61}$, P. Moskvitina ${ }^{25}$, J. Moss ${ }^{124,273}$, E. J. W. Moyse ${ }^{3}$, S. Muanza1, J. Mueller ${ }^{108}$, R. S. P. Mueller ${ }^{83}$, D. Muenstermann ${ }^{118}$, G. A. Mullier ${ }^{36}$, D. P. Mungo ${ }^{47,66}$, J. L. Munoz Martinez ${ }^{145}$, F. J. Munoz Sanchez ${ }^{142}$, P. Murin ${ }^{155}$, W. J. Murray 21,127, A. Murrone ${ }^{47,66}$, J. M. Muse ${ }^{2}$, M. Muškinja ${ }^{32}$, C. Mwewa93, A. G. Myagkov ${ }^{148,252}$, A. A. Myers ${ }^{108}$, G. Myers ${ }^{160}$, J. Myers ${ }^{48}$, M. Myska ${ }^{44}$, B. P. Nachman ${ }^{32}$, O. Nackenhorst ${ }^{188}$, A. Nag Nag ${ }^{136}$, K. Nagai ${ }^{56}$, K. Nagano ${ }^{76}$, Y. Nagasaka ${ }^{236}$, J. L. Nagle ${ }^{91}$, E. Nagy ${ }^{1}$, A. M. Nairz ${ }^{4}$, Y. Nakahama ${ }^{84}$, K. Nakamura76, T. Nakamura ${ }^{86}$, H. Nanjo ${ }^{220}$, F. Napolitano ${ }^{67}$, R. F. Naranjo Garcia ${ }^{58}$, R. Narayan ${ }^{137}$, I. Naryshkin ${ }^{117}$, M. Naseri131, T. Naumann ${ }^{58}$, G. Navarro ${ }^{235}$, P. Y. Nechaeva ${ }^{37}$, F. Nechansky ${ }^{58}$, T. J. Neep ${ }^{49}$, A. Negri ${ }^{199,200}$, M. Negrini ${ }^{39}$, C. Nellist ${ }^{25}$, C. Nelson ${ }^{31}$, M. E. Nelson ${ }^{64,65}$, S. Nemecek ${ }^{180}$, M. Nessi ${ }^{4,274}$, M. S. Neubauer ${ }^{95}$, F. Neuhaus ${ }^{15}$, M. Neumann ${ }^{97}$, R. Newhouse ${ }^{185}$, P. R. Newman ${ }^{49}$, C. W. Ng ${ }^{108}$, Y. S. Ng ${ }^{96}$, Y. W. Y. Ng ${ }^{82}$, B. Ngair ${ }^{120}$, H. D. N. Nguyen ${ }^{1}$ T. Nguyen Manh ${ }^{80}$, E. Nibigira ${ }^{24}$, R. B. Nickerson ${ }^{56}$, R. Nicolaidou ${ }^{61}$, D. S. Nielsen ${ }^{8}$, J. Nielsen ${ }^{22}$, M. Niemeyer ${ }^{5}$, N. Nikiforou ${ }^{62}$, V. Nikolaenko ${ }^{148,252}$, I. Nikolic-Audit ${ }^{125}$, K. Nikolopoulos ${ }^{49}$, P. Nilsson ${ }^{91}$, H. R. Nindhito ${ }^{20}$, A. Nisati ${ }^{75}$, N. Nishu ${ }^{196}$, R. Nisius ${ }^{114}$, I. Nitsche ${ }^{188}$, T. Nitta ${ }^{225}$, T. Nobe ${ }^{86}$, D. L. Noel ${ }^{121}$, Y. Noguchi ${ }^{35}$, I. Nomidis ${ }^{125}$, M. A. Nomura ${ }^{91}$, M. Nordberg ${ }^{4}$, J. Novak ${ }^{181}$, T. Novak ${ }^{181}$, O. Novgorodova ${ }^{136}$, R. Novotny ${ }^{214}$, L. Nozka170, K. Ntekas ${ }^{82}$, E. Nurse ${ }^{113}$, F. G. Oakham ${ }^{131,247}$, J. Ocariz ${ }^{125}$, A. Ochi' ${ }^{219}$, I. Ochoa ${ }^{27}$, J. P. Ochoa-Ricoux ${ }^{193}$, K. O'Connor ${ }^{134}$, S. Oda ${ }^{226}$, S. Odaka ${ }^{76}$, S. Oerdek ${ }^{5}$, A. Ogrodnik ${ }^{17}$, A. Oh ${ }^{142}$, C. C. Ohm ${ }^{233}$, H. Oide ${ }^{87}$, R. Oishi ${ }^{86}$, M. L. Ojeda7, H. Okawa ${ }^{216}$, Y. Okazaki ${ }^{35}$, M. W. O'Keefe ${ }^{147}$, Y. Okumura ${ }^{86}$, A. Olariu ${ }^{41}$, L. F. Oleiro Seabra ${ }^{27}$, S. A. Olivares Pino' ${ }^{193}$, D. Oliveira Damazio ${ }^{91}$, J. L. Oliver ${ }^{197}$, M. J. R. Olsson ${ }^{82}$, A. Olszewski ${ }^{101}$, J. Olszowska ${ }^{101}$, Ö. O. Öncel ${ }^{123}$, D. C. O'Neil ${ }^{102}$, A. P. O'neill ${ }^{56}$, A. Onofre ${ }^{27,168}$, P. U. E. Onyisi ${ }^{62}$, H. Oppen ${ }^{144}$, R. G. Oreamuno Madriz ${ }^{18}$, M. J. Oreglia ${ }^{205}$, G. E. Orellana ${ }^{52}$, D. Orestano ${ }^{141,161}$, N. Orlando ${ }^{145}$, R. S. Orr7, V. O'Shea ${ }^{119}$, R. Ospanov ${ }^{116}$, G. Otero y Garzon ${ }^{189}$, H. Otono ${ }^{226}$, P. S. Ott ${ }^{67}$, G. J. Ottino ${ }^{32}$, M. Ouchrif ${ }^{90}$, J. Ouellette ${ }^{91}$, F. Ould-Saada ${ }^{144}$, A. Ouraou ${ }^{61283}$, Q. Ouyang ${ }^{98}$, M. Owen ${ }^{119}$, R. E. Owen ${ }^{21}$, V. E. Ozcan ${ }^{19}$, N. Ozturk ${ }^{105}$, J. Pacalt ${ }^{170}$, H. A. Pacey ${ }^{121}$, K. Pachal ${ }^{79}$, A. Pacheco Pages ${ }^{145}$, C. Padilla Aranda145, S. Pagan Griso ${ }^{32}$, 
G. Palacino ${ }^{160}$, S. Palazzo ${ }^{167}$, S. Palestini ${ }^{4}$, M. Palka ${ }^{207}$, P. Palni ${ }^{17}$, C. E. Pandini ${ }^{20}$, J. G. Panduro Vazquez ${ }^{6}$, P. Pani ${ }^{58}$, G. Panizzo ${ }^{13,182}$, L. Paolozzi ${ }^{20}$, C. Papadatos ${ }^{80}$, K. Papageorgiou ${ }^{68,259}$, S. Parajuli137,

A. Paramonov ${ }^{12}$, C. Paraskevopoulos ${ }^{42}$, D. Paredes Hernandez ${ }^{232}$, S. R. Paredes Saenz ${ }^{56}$, B. Parida ${ }^{107}$, T. H. Park7 , A. J. Parker ${ }^{124}$, M. A. Parker ${ }^{121}$, F. Parodi ${ }^{111,12}$, E. W. Parrish ${ }^{18}$, J. A. Parsons ${ }^{69}$, U. Parzefall ${ }^{81}$,

L. Pascual Dominguez ${ }^{125}$, V. R. Pascuzzi ${ }^{32}$, J. M. P. Pasner ${ }^{22}$, F. Pasquali43, E. Pasqualucci75,

S. Passaggio ${ }^{112}$, F. Pastore ${ }^{6}$, P. Pasuwan ${ }^{64,65}$, S. Pataraia ${ }^{15}$, J. R. Pater ${ }^{142}$, A. Pathak ${ }^{109,248}$, J. Patton ${ }^{147}$,

T. Pauly4 , J. Pearkes ${ }^{115}$, M. Pedersen ${ }^{144}$, L. Pedraza Diaz ${ }^{25}$, R. Pedro ${ }^{27}$, T. Peiffer ${ }^{5}$, S. V. Peleganchuk ${ }^{70,71}$, O. Penc ${ }^{180}$, C. Peng ${ }^{232}$, H. Peng ${ }^{116}$, B. S. Peralva ${ }^{78}$, M. M. Perego ${ }^{23}$, A. P. Pereira Peixoto ${ }^{27}$,

L. Pereira Sanchez ${ }^{64,65}$, D. V. Perepelitsa91, E. Perez Codina ${ }^{166}$, L. Perini ${ }^{47,66}$, H. Pernegger ${ }^{4}$, S. Perrella ${ }^{4}$, A. Perrevoort ${ }^{43}$, K. Peters ${ }^{58}$, R. F. Y. Peters ${ }^{142}$, B. A. Petersen ${ }^{4}$, T. C. Petersen ${ }^{8}$, E. Petit ${ }^{1}$, V. Petousis ${ }^{44}$, C. Petridou 99 , F. Petrucci ${ }^{141,161}$, M. Pettee ${ }^{103}$, N. E. Pettersson ${ }^{3}$, K. Petukhova ${ }^{165}$, A. Peyaud ${ }^{61}$, R. Pezoa ${ }^{154}$, L. Pezzotti ${ }^{199,200}$, T. Pham ${ }^{110}$, P. W. Phillips ${ }^{21}$, M. W. Phipps ${ }^{95}$, G. Piacquadio ${ }^{130}$, E. Pianori32, A. Picazio3, R. H. Pickles ${ }^{142}$, R. Piegaia' ${ }^{189}$, D. Pietreanu ${ }^{41}$, J. E. Pilcher ${ }^{205}$, A. D. Pilkington ${ }^{142}$, M. Pinamonti ${ }^{13,182}$, J. L. Pinfold ${ }^{143}$, C. Pitman Donaldson ${ }^{113}$, M. Pitt ${ }^{10}$, L. Pizzimento ${ }^{33,34}$, A. Pizzini ${ }^{43}$, M.-A. Pleier ${ }^{91}$, V. Plesanovs ${ }^{81}$, V. Pleskot ${ }^{165}$, E. Plotnikova ${ }^{30}$, P. Podberezko ${ }^{70,71}$, R. Poettgen ${ }^{36}$, R. Poggi ${ }^{20}$, L. Poggioli ${ }^{125}$, I. Pogrebnyak ${ }^{153}$, D. Pohl ${ }^{123}$, I. Pokharel ${ }^{5}$, G. Polesello ${ }^{199}$, A. Poley ${ }^{102,166}$, A. Policicchio ${ }^{75,100}$, R. Polifka ${ }^{165}$, A. Polini ${ }^{39}$, C. S. Pollard ${ }^{58}$, V. Polychronakos ${ }^{91}$, D. Ponomarenko ${ }^{132}$, L. Pontecorvo ${ }^{4}$, S. Popa ${ }^{237}$, G. A. Popeneciu ${ }^{238}$, L. Portales ${ }^{16}$, D. M. Portillo Quintero ${ }^{183}$, S. Pospisil ${ }^{44}$, K. Potamianos ${ }^{58}$, I. N. Potrap ${ }^{30}$, C. J. Potter ${ }^{121}$, H. Potti ${ }^{62}$, T. Poulsen ${ }^{36}$, J. Poveda ${ }^{77}$, T. D. Powell ${ }^{60}$, G. Pownall ${ }^{58}$, M. E. Pozo Astigarraga ${ }^{4}$, A. Prades Ibanez ${ }^{77}$, P. Pralavorio ${ }^{1}$, M. M. Prapa ${ }^{198}$, S. Prell ${ }^{59}$, D. Price ${ }^{142}$, M. Primavera ${ }^{177}$, M. L. Proffitt ${ }^{53}$, N. Proklova ${ }^{132}$, K. Prokoév ${ }^{231}$, F. Prokoshin ${ }^{30}$, S. Protopopescu ${ }^{91}$, J. Proudfoot ${ }^{12}$, M. Przybycien ${ }^{17}$, D. Pudzha ${ }^{117}$, A. Puri" ${ }^{95}$, P. Puzo ${ }^{23}$, D. Pyatiizbyantseva ${ }^{132}$, J. Qian ${ }^{57}$, Y. Qin ${ }^{142}$, A. Quadt M. Queitsch-Maitland4, G. Rabanal Bolanos ${ }^{88}$, M. Racko92, F. Ragusa ${ }^{47,66}$, G. Rahal ${ }^{239}$, J. A. Raine ${ }^{20}$, S. Rajagopalan ${ }^{91}$, A. Ramirez Morales ${ }^{138}$, K. Ran ${ }^{98,179}$, D. F. Rassloff67, D. M. Rauch ${ }^{58}$, F. Rauscher ${ }^{83}$, S. Rave ${ }^{15}$, B. Ravina ${ }^{119}$, I. Ravinovich ${ }^{107}$, J. H. Rawling ${ }^{142}$, M. Raymond ${ }^{4}$, A. L. Read ${ }^{144}$, N. P. Readioff ${ }^{60}$, M. Reale ${ }^{177,210}$, D. M. Rebuzzi ${ }^{199,200}$, G. Redlinger ${ }^{91}$, K. Reeves ${ }^{201}$, D. Reikher ${ }^{10}$, A. Reiss ${ }^{15}$, A. Rej ${ }^{122}$, C. Rembser ${ }^{4}$, A. Renardi ${ }^{58}$, M. Renda ${ }^{41}$, M. B. Rendel ${ }^{114}$, A. G. Rennie ${ }^{119}$, S. Resconi ${ }^{47}$, E. D. Resseguie ${ }^{32}$, S. Rettie ${ }^{113}$, B. Reynolds ${ }^{149}$, E. Reynolds ${ }^{49}$, O. L. Rezanova70,71, P. Reznicek ${ }^{165}$, E. Ricci' ${ }^{191,192}$, R. Richter ${ }^{114}$, S. Richter ${ }^{58}$, E. Richter-Was ${ }^{207}$, M. Ridel ${ }^{125}$, P. Rieck ${ }^{114}$, O. Rifki ${ }^{58}$, M. Rijssenbeek ${ }^{130}$, A. Rimoldi ${ }^{199,200}$, M. Rimoldi ${ }^{58}$, L. Rinaldi ${ }^{39}$, T. T. Rinn ${ }^{95}$, G. Ripellino ${ }^{233}$, I. Riu ${ }^{145}$, P. Rivadeneira ${ }^{58}$, J. C. Rivera Vergara ${ }^{40}$, F. Rizatdinova ${ }^{73}$, E. Rizvi ${ }^{138}$, C. Rizzi ${ }^{4}$, S. H. Robertson ${ }^{31,255}$, M. Robin ${ }^{58}$, D. Robinson ${ }^{121}$, C. M. Robles Gajardo ${ }^{154}$, M. Robles Manzano ${ }^{15}$, A. Robson ${ }^{119}$, A. Rocchi ${ }^{33,34}$, C. Roda72,140, S. Rodriguez Bosca ${ }^{77}$, A. Rodriguez Rodriguez ${ }^{81}$, A. M. Rodríguez Vera ${ }^{190}$, S. Roe ${ }^{4}$, J. Roggel ${ }^{97}$, O. Røhne ${ }^{144}$, R. Röhrig ${ }^{114}$, R. A. Rojas ${ }^{154}$, B. Roland ${ }^{81}$, C. P. A. Roland ${ }^{160}$, J. Roloff ${ }^{91}$, A. Romaniouk ${ }^{132}$, M. Romano ${ }^{38,39}$, N. Rompotis ${ }^{147}$, M. Ronzani ${ }^{186}$, L. Roos ${ }^{125}$, S. Rosati ${ }^{75}$, G. Rosin ${ }^{3}$, B. J. Rosser ${ }^{173}$, E. Rossi ${ }^{58}$, E. Rossi ${ }^{141,161}$, E. Rossi ${ }^{50,51}$, L. P. Rossi ${ }^{112}$, L. Rossini ${ }^{58}$, R. Rosten ${ }^{145}$, M. Rotaru ${ }^{41}$, B. Rottler ${ }^{81}$, D. Rousseau ${ }^{23}$, G. Rovelli ${ }^{199,200}$, A. Roy ${ }^{62}$, D. Roy ${ }^{178}$, A. Rozanov1, Y. Rozen ${ }^{11}$, X. Ruan ${ }^{178}$, T. A. Ruggeri ${ }^{197}$, F. Rühr ${ }^{81}$, A. Ruiz-Martinez ${ }^{77}$, A. Rummler ${ }^{4}$, Z. Rurikova ${ }^{81}$, N. A. Rusakovich ${ }^{30}$, H. L. Russell ${ }^{31}$, L. Rustige ${ }^{24,188}$, J. P. Rutherfoord ${ }^{135}$, E. M. Rüttinger ${ }^{60}$, M. Rybar ${ }^{165}$, G. Rybkin ${ }^{23}$, E. B. Rye ${ }^{144}$, A. Ryzhov ${ }^{148}$, J. A. Sabater Iglesias ${ }^{58}$, P. Sabatini ${ }^{77}$, L. Sabetta ${ }^{75,100}$, S. Sacerdoti ${ }^{23}$, H. F-W. Sadrozinski22, R. Sadykov ${ }^{30}$, F. Safai Tehrani ${ }^{75}$, B. Safarzadeh Samani ${ }^{9}$, M. Safdari ${ }^{115}$, P. Saha ${ }^{18}$, S. Saha ${ }^{31}$, M. Sahinsoy ${ }^{114}$, A. Sahu ${ }^{97}$, M. Saimpert ${ }^{4}$, M. Saito ${ }^{86}$, T. Saito ${ }^{86}$, H. Sakamoto ${ }^{86}$, D. Salamani ${ }^{20}$, G. Salamanna ${ }^{141,161}$, A. Salnikov ${ }^{115}$, J. Salt ${ }^{77}$, A. Salvador Salas ${ }^{145}$, D. Salvatore ${ }^{163,164}$, F. Salvatore ${ }^{9}$, A. Salvucci ${ }^{175}$, A. Salzburger ${ }^{4}$, 
J. Samarati ${ }^{4}$, D. Sammel ${ }^{81}$, D. Sampsonidis ${ }^{99}$, D. Sampsonidou ${ }^{152,196}$, J. Sánchez ${ }^{77}$,

A. Sanchez Pineda ${ }^{4,13,182}$, H. Sandaker ${ }^{144}$, C. O. Sander ${ }^{58}$, I. G. Sanderswood ${ }^{118}$, M. Sandhoff ${ }^{97}$,

C. Sandoval ${ }^{240}$, D. P. C. Sankey ${ }^{21}$, M. Sannino ${ }^{111,112}$, Y. Sano ${ }^{84}$, A. Sansoni ${ }^{74}$, C. Santoni ${ }^{24}$, H. Santos ${ }^{27,209}$,

S. N. Santpur ${ }^{32}$, A. Santra ${ }^{77}$, K. A. Saoucha ${ }^{60}$, A. Sapronov ${ }^{30}$, J. G. Saraiva ${ }^{27,234}$, O. Sasaki ${ }^{76}$, K. Sato ${ }^{216}$,

F. Sauerburger ${ }^{81}$, E. Sauvan ${ }^{16}$, P. Savard ${ }^{7,247}$, R. Sawada ${ }^{86}$, C. Sawyer ${ }^{21}$, L. Sawyer ${ }^{241}$, I. Sayago Galvan ${ }^{77}$,

C. Sbarra ${ }^{39}$, A. Sbrizzi ${ }^{13,182}$, T. Scanlon ${ }^{113}$, J. Schaarschmidt ${ }^{53}$, P. Schacht ${ }^{114}$, D. Schaefer ${ }^{205}$, L. Schaefer ${ }^{173}$,

U. Schäfer ${ }^{15}$, A. C. Schaffer ${ }^{23}$, D. Schaile ${ }^{83}$, R. D. Schamberger ${ }^{130}$, E. Schanet ${ }^{83}$, C. Scharf ${ }^{96}$,

N. Scharmberg ${ }^{142}$, V. A. Schegelsky ${ }^{117}$, D. Scheirich ${ }^{165}$, F. Schenck ${ }^{96}$, M. Schernau ${ }^{82}$, C. Schiavi ${ }^{111,112}$,

L. K. Schildgen ${ }^{123}$, Z. M. Schillaci'134, E. J. Schioppa ${ }^{177,210}$, M. Schioppa ${ }^{163,164}$, K. E. Schleicher ${ }^{81}$,

S. Schlenker ${ }^{4}$, K. R. Schmidt-Sommerfeld ${ }^{114}$, K. Schmieden ${ }^{15}$, C. Schmitt ${ }^{15}$, S. Schmitt ${ }^{58}$, L. Schoeffel ${ }^{61}$,

A. Schoening ${ }^{146}$, P. G. Scholer ${ }^{81}$, E. Schopf ${ }^{56}$, M. Schott ${ }^{15}$, J. F. P. Schouwenberg ${ }^{25}$, J. Schovancova ${ }^{4}$,

S. Schramm ${ }^{20}$, F. Schroeder ${ }^{97}$, A. Schulte ${ }^{15}$, H-C. Schultz-Coulon ${ }^{67}$, M. Schumacher ${ }^{81}$, B. A. Schumm ${ }^{22}$,

Ph. Schune ${ }^{61}$, A. Schwartzman ${ }^{115}$, T. A. Schwarz ${ }^{57}$, Ph. Schwemling ${ }^{61}$, R. Schwienhorst ${ }^{153}$, A. Sciandra ${ }^{22}$,

G. Sciolla ${ }^{134}$, F. Scuri ${ }^{72}$, F. Scutti ${ }^{110}$, L. M. Scyboz ${ }^{114}$, C. D. Sebastiani ${ }^{147}$, K. Sedlaczek ${ }^{188}$, P. Seema ${ }^{96}$,

S. C. Seidel ${ }^{214}$, A. Seiden ${ }^{22}$, B. D. Seidlitz ${ }^{91}$, T. Seiss ${ }^{205}$, C. Seitz ${ }^{58}$, J. M. Seixas ${ }^{55}$, G. Sekhniaidze ${ }^{50}$,

S. J. Sekula ${ }^{137}$, N. Semprini-Cesari ${ }^{38,39}$, S. Sen ${ }^{79}$, C. Serfon ${ }^{91}$, L. Serin ${ }^{23}$, L. Serkin ${ }^{13,14}$, M. Sessa ${ }^{116}$,

H. Severini' ${ }^{2}$, S. Sevova ${ }^{115}$, F. Sforza ${ }^{111,112}$, A. Sfyrla ${ }^{20}$, E. Shabalina ${ }^{5}$, J. D. Shahinian ${ }^{173}$, N. W. Shaikh ${ }^{64,65}$,

D. Shaked Renous ${ }^{107}$, L. Y. Shan ${ }^{98}$, M. Shapiro ${ }^{32}$, A. Sharma ${ }^{4}$, A. S. Sharma ${ }^{197}$, P. B. Shatalov ${ }^{206}$,

K. Shaw ${ }^{9}$, S. M. Shaw ${ }^{142}$, M. Shehade ${ }^{107}$, Y. Shen ${ }^{2}$, A. D. Sherman ${ }^{157}$, P. Sherwood ${ }^{113}$, L. Shi" ${ }^{113}$,

C. O. Shimmin ${ }^{103}$, Y. Shimogama ${ }^{225}$, M. Shimojima ${ }^{204}$, J. D. Shinner6, I. P. J. Shipsey ${ }^{56}$, S. Shirabe ${ }^{87}$,

M. Shiyakova ${ }^{30,275}$, J. Shlomi ${ }^{107}$, A. Shmeleva ${ }^{37}$, M. J. Shochet ${ }^{205}$, J. Shojaii ${ }^{110}$, D. R. Shope ${ }^{233}$,

S. Shrestha ${ }^{149}$, E. M. Shrif ${ }^{178}$, M. J. Shroff ${ }^{40}$, E. Shulga ${ }^{107}$, P. Sicho ${ }^{180}$, A. M. Sickles ${ }^{95}$,

E. Sideras Haddad ${ }^{178}$, O. Sidiropoulou ${ }^{4}$, A. Sidoti ${ }^{38,39}$, F. Siegert ${ }^{136}$, Dj. Sijacki ${ }^{104}$, M. Jr. Silva ${ }^{109}$,

M. V. Silva Oliveira4 ${ }^{4}$ S. B. Silverstein ${ }^{64}$, S. Simion ${ }^{23}$, R. Simoniello ${ }^{15}$, C. J. Simpson-allsop ${ }^{49}$, S. Simsek ${ }^{169}$,

P. Sinervo ${ }^{7}$, V. Sinetckii ${ }^{208}$, S. Singh ${ }^{102}$, S. Sinha ${ }^{178}$, M. Sioli ${ }^{38,39}$, I. Siral ${ }^{48}$, S. Yu. Sivoklokov ${ }^{208}$,

J. Sjölin ${ }^{64,65}$, A. Skaf5 ${ }^{5}$ E. Skorda ${ }^{36}$, P. Skubic ${ }^{2}$, M. Slawinska ${ }^{101}$, K. Sliwa ${ }^{126}$, V. Smakhtin ${ }^{107}$, B. H. Smart ${ }^{21}$,

J. Smiesko ${ }^{155}$, N. Smirnov ${ }^{132}$, S. Yu. Smirnov ${ }^{132}$, Y. Smirnov ${ }^{132}$, L. N. Smirnova ${ }^{208,276}$, O. Smirnova ${ }^{36}$,

E. A. Smith ${ }^{205}$, H. A. Smith ${ }^{56}$, M. Smizanska ${ }^{118}$, K. Smolek ${ }^{44}$, A. Smykiewicz ${ }^{101}$, A. A. Snesarev ${ }^{37}$,

H. L. Snoek ${ }^{43}$, I. M. Snyder ${ }^{48}$, S. Snyder ${ }^{91}$, R. Sobie ${ }^{40,255}$, A. Soffer ${ }^{10}$, A. Søgaard ${ }^{167}$, F. Sohns ${ }^{5}$,

C. A. Solans Sanchez ${ }^{4}$, E. Yu. Soldatov ${ }^{132}$, U. Soldevila77, A. A. Solodkov ${ }^{148}$, A. Soloshenko ${ }^{30}$,

O. V. Solovyanov ${ }^{148}$, V. Solovyev ${ }^{117}$, P. Sommer ${ }^{60}$, H. Son ${ }^{126}$, A. Sonay ${ }^{145}$, W. Song ${ }^{21}$, W. Y. Song ${ }^{190}$,

A. Sopczak ${ }^{44}$, A. L. Sopio ${ }^{113}$, F. Sopkova ${ }^{155}$, S. Sottocornola ${ }^{199,200}$, R. Soualah ${ }^{13,182}$, A. M. Soukharev ${ }^{70,71}$,

D. South ${ }^{58}$, S. Spagnolo ${ }^{177,210}$, M. Spalla ${ }^{114}$, M. Spangenberg ${ }^{127}$, F. Spanò ${ }^{6}$, D. Sperlich ${ }^{81}$, T. M. Spieker ${ }^{67}$,

G. Spigo ${ }^{4}$, M. Spina9 , D. P. Spiteri ${ }^{119}$, M. Spousta ${ }^{165}$, A. Stabile ${ }^{47,66}$, B. L. Stamas ${ }^{18}$, R. Stamen ${ }^{67}$,

M. Stamenkovic ${ }^{43}$, A. Stampekis ${ }^{49}$, E. Stanecka ${ }^{101}$, B. Stanislaus ${ }^{56}$, M. M. Stanitzki ${ }^{58}$, M. Stankaityte ${ }^{56}$,

B. Stapf ${ }^{43}$, E. A. Starchenko ${ }^{148}$, G. H. Stark ${ }^{22}$, J. Stark ${ }^{183}$, P. Staroba ${ }^{180}$, P. Starovoitov ${ }^{67}$, S. Stärz ${ }^{31}$,

R. Staszewski ${ }^{101}$, G. Stavropoulos ${ }^{198}$, M. Stegler ${ }^{58}$, P. Steinberg ${ }^{91}$, A. L. Steinhebel ${ }^{48}$, B. Stelzer ${ }^{102,166}$,

H. J. Stelzer ${ }^{108}$, O. Stelzer-Chilton ${ }^{166}$, H. Stenzel ${ }^{158}$, T. J. Stevenson ${ }^{9}$, G. A. Stewart ${ }^{4}$, M. C. Stockton ${ }^{4}$,

G. Stoicea ${ }^{41}$, M. Stolarski' ${ }^{27}$, S. Stonjek ${ }^{114}$, A. Straessner ${ }^{136}$, J. Strandberg ${ }^{233}$, S. Strandberg ${ }^{64,65}$,

M. Strauss ${ }^{2}$, T. Strebler ${ }^{1}$, P. Strizenec ${ }^{155}$, R. Ströhmer ${ }^{139}$, D. M. Strom ${ }^{48}$, R. Stroynowski ${ }^{137}$, A. Strubig ${ }^{64,65}$,

S. A. Stucci ${ }^{91}$, B. Stugu ${ }^{156}$, J. Stupak ${ }^{2}$, N. A. Styles ${ }^{58}$, D. Su ${ }^{115}$, W. Su ${ }^{53,152,196}$, X. Su ${ }^{116}$, N. B. Suarez ${ }^{108}$,

V. V. Sulin ${ }^{37}$, M. J. Sullivan ${ }^{147}$, D. M. S. Sultan ${ }^{20}$, S. Sultansoy ${ }^{242}$, T. Sumida ${ }^{35}$, S. Sun ${ }^{57}$, X. Sun ${ }^{142}$,

C. J. E. Suster ${ }^{106}$, M. R. Sutton ${ }^{9}$, S. Suzuki ${ }^{76}$, M. Svatos ${ }^{180}$, M. Swiatlowski ${ }^{166}$, S. P. Swift ${ }^{223}$, T. Swirski ${ }^{139}$, 
A. Sydorenko ${ }^{15}$, I. Sykora ${ }^{92}$, M. Sykora ${ }^{165}$, T. Sykora ${ }^{165}$, D. Ta ${ }^{15}$, K. Tackmann ${ }^{58,277}$, J. Taenzer ${ }^{10}$, A. Taffard ${ }^{82}$, R. Tafirout ${ }^{166}$, E. Tagiev ${ }^{148}$, R. H. M. Taibah ${ }^{125}$, R. Takashima ${ }^{243}$, K. Takeda ${ }^{219}$, T. Takeshita ${ }^{218}$, E. P. Takeva ${ }^{167}$, Y. Takubo ${ }^{76}$, M. Talby ${ }^{1}$, A. A. Talyshev70,71, K. C. Tam ${ }^{232}$, N. M. Tamir ${ }^{10}$, J. Tanaka ${ }^{86}$, R. Tanaka ${ }^{23}$, S. Tapia Araya95, S. Tapprogge ${ }^{15}$, A. Tarek Abouelfadl Mohamed ${ }^{153}$, S. Tarem ${ }^{11}$, K. Tariq $^{187}$, G. Tarna ${ }^{41,278}$, G. F. Tartarelli ${ }^{47}$, P. Tas ${ }^{165}$, M. Tasevsky ${ }^{180}$, E. Tassi ${ }^{163,164}$, G. Tateno ${ }^{86}$, A. Tavares Delgado ${ }^{27}$, Y. Tayalati ${ }^{120}$, A. J. Taylor ${ }^{167}$, G. N. Taylor ${ }^{110}$, W. Taylor ${ }^{190}$, H. Teagle ${ }^{147}$, A. S. Tee ${ }^{118}$, R. Teixeira De Lima ${ }^{115}$,

P. Teixeira-Dias ${ }^{6}$, H. Ten Kate ${ }^{4}$, J. J. Teoh ${ }^{43}$, K. Terashi ${ }^{86}$, J. Terron ${ }^{54}$, S. Terzo ${ }^{145}$, M. Testa ${ }^{74}$, R. J. Teuscher ${ }^{7,255}$, N. Themistokleous ${ }^{167}$, T. Theveneaux-Pelzer ${ }^{96}$, D. W. Thomas ${ }^{6}$, J. P. Thomas ${ }^{49}$, E. A. Thompson ${ }^{58}$, P. D. Thompson ${ }^{49}$, E. Thomson ${ }^{173}$, E. J. Thorpe ${ }^{138}$, V. O. Tikhomirov ${ }^{37,279}$, Yu. A. Tikhonov ${ }^{70,71}$, S. Timoshenko ${ }^{132}$, P. Tipton ${ }^{103}$, S. Tisserant', K. Todome ${ }^{38,39}$, S. Todorova-Nova ${ }^{165}$, S. Todt ${ }^{136}$, J. Tojo ${ }^{226}$, S. Tokár ${ }^{92}$, K. Tokushuku ${ }^{76}$, E. Tolley ${ }^{149}$, R. Tombs ${ }^{121}$, K. G. Tomiwa ${ }^{178}$, M. Tomoto ${ }^{76,84}$, L. Tompkins ${ }^{115}$, P. Tornambe ${ }^{3}$, E. Torrence ${ }^{48}$, H. Torres ${ }^{136}$, E. Torró Pastor ${ }^{77}$, M. Toscani ${ }^{189}$, C. Tosciri ${ }^{56}$, J. Toth ${ }^{1,280}$, D. R. Tovey ${ }^{60}$, A. Traeet ${ }^{156}$, C. J. Treado ${ }^{186}$, T. Trefzger ${ }^{139}$, F. Tresoldi ${ }^{9}$, A. Tricoli ${ }^{91}$, I. M. Trigger ${ }^{166}$, S. Trincaz-Duvoid ${ }^{125}$, D. A. Trischuk ${ }^{185}$, W. Trischuk7, B. Trocmé183, A. Trofymov' ${ }^{23}$, C. Troncon ${ }^{47}$, F. Trovato ${ }^{9}$, L. Truong ${ }^{150}$, M. Trzebinski ${ }^{101}$, A. Trzupek ${ }^{101}$, F. Tsai ${ }^{58}$, P. V. Tsiareshka ${ }^{217,268,}$ A. Tsirigotis ${ }^{99,269}$, V. Tsiskaridze ${ }^{130}$, E. G. Tskhadadze ${ }^{224}$, M. Tsopoulou ${ }^{99}$, I. I. Tsukerman ${ }^{206}$, V. Tsulaia ${ }^{32}$, S. Tsuno ${ }^{76}$, D. Tsybychev ${ }^{130}$, Y. Tu ${ }^{232}$, A. Tudorache ${ }^{41}$, V. Tudorache ${ }^{41}$, A. N. Tuna ${ }^{4}$, S. Turchikhin ${ }^{30}$, D. Turgeman ${ }^{107}$, I. Turk Cakir ${ }^{228,281}$, R. J. Turner ${ }^{49}$, R. Turra ${ }^{47}$, P. M. Tuts ${ }^{69}$, S. Tzamarias ${ }^{99}{ }^{,}$E. Tzovara ${ }^{15}$, K. Uchida ${ }^{86}$, F. Ukegawa ${ }^{216}$, G. Unal ${ }^{4}$, M. Unal ${ }^{62}$, A. Undrus ${ }^{91}$, G. Unel ${ }^{82}$, F. C. Ungaro ${ }^{110}$, Y. Unno ${ }^{76}$, K. Uno ${ }^{86}$, J. Urban ${ }^{155}$, P. Urquijo ${ }^{110}$, G. Usai ${ }^{105}$, Z. Uysal' ${ }^{128}$, V. Vacek ${ }^{44}$, B. Vachon ${ }^{31}$, K. O. H. Vadla ${ }^{144}$, T. Vafeiadis ${ }^{4}$, A. Vaidya ${ }^{113}$, C. Valderanis ${ }^{83}$, E. Valdes Santurio ${ }^{64,65}$, M. Valente ${ }^{166}$, S. Valentinetti ${ }^{38,39}$, A. Valero ${ }^{77}$, L. Valéry ${ }^{58}$, R. A. Vallance ${ }^{49}$, A. Vallier ${ }^{4}$, J. A. Valls Ferrer ${ }^{77}$, T. R. Van Daalen ${ }^{145}$, P. Van Gemmeren ${ }^{12}$, S. Van Stroud ${ }^{113}$, I. Van Vulpen ${ }^{43}$, M. Vanadia ${ }^{33,34}$, W. Vandelli4, M. Vandenbroucke ${ }^{61}$, E. R. Vandewall73, D. Vannicola ${ }^{75,100}$, R. Vari ${ }^{75}$, E. W. Varnes ${ }^{135}$, C. Varni ${ }^{111,112}$, T. Varol ${ }^{45}$, D. Varouchas ${ }^{23}$, K. E. Varvell ${ }^{106}$, M. E. Vasile ${ }^{41}$, G. A. Vasquez ${ }^{40}$, F. Vazeille ${ }^{24}$,

D. Vazquez Furelos ${ }^{145}$, T. Vazquez Schroeder ${ }^{4}$, J. Veatch ${ }^{5}$, V. Vecchio ${ }^{142}$, M. J. Veen ${ }^{43}$, L. M. Veloce ${ }^{7}$, F. Veloso ${ }^{27,202}$, S. Veneziano ${ }^{75}$, A. Ventura ${ }^{177,210}$, A. Verbytskyi ${ }^{114}$, V. Vercesi ${ }^{199}$, M. Verducci ${ }^{72,140}$, C. M. Vergel Infante ${ }^{59}$, C. Vergis ${ }^{123}$, W. Verkerke ${ }^{43}$, A. T. Vermeulen ${ }^{43}$, J. C. Vermeulen ${ }^{43}$, C. Vernieri ${ }^{115}$, P. J. Verschuuren ${ }^{6}$, M. C. Vetterli ${ }^{102,247}$, N. Viaux Maira ${ }^{154}$, T. Vickey ${ }^{60}$, O. E. Vickey Boeriu ${ }^{60}$, G. H. A. Viehhauser ${ }^{56}$, L. Vigani ${ }^{146}$, M. Villa ${ }^{38,39}$, M. Villaplana Perez ${ }^{77}$, E. M. Villhauer ${ }^{167}$, E. Vilucchi ${ }^{74}$, M. G. Vincter ${ }^{131}$, G. S. Virdee ${ }^{49}$, A. Vishwakarma ${ }^{167}$, C. Vittori ${ }^{38,39}$, I. Vivarelli ${ }^{9}$, M. Vogel $^{97}$, P. Vokac ${ }^{44}$, J. Von Ahnen ${ }^{58}$, S. E. von Buddenbrock ${ }^{178}$, E. Von Toerne ${ }^{123}$, V. Vorobel ${ }^{165}$, K. Vorobev ${ }^{132}$, M. Vos ${ }^{77}$, J. H. Vossebeld ${ }^{147}$, M. Vozak ${ }^{142}$, N. Vranjes ${ }^{104}$, M. Vranjes Milosavljevic ${ }^{104}$, V. Vrba ${ }^{44}$, M. Vreeswijk ${ }^{43}$, N. K. Vu', R. Vuillermet ${ }^{4}$, I. Vukotic ${ }^{205}$, S. Wada ${ }^{216}$, P. Wagner ${ }^{123}$, W. Wagner ${ }^{97}$, J. Wagner-Kuhr ${ }^{83}$, S. Wahdan ${ }^{97}$, H. Wahlberg ${ }^{52}$, R. Wakasa ${ }^{216}$, V. M. Walbrecht ${ }^{114}$, J. Walder ${ }^{21}$, R. Walker ${ }^{83}$, S. D. Walker ${ }^{6}$, W. Walkowiak ${ }^{122}$, V. Wallangen ${ }^{64,65}$, A. M. Wang ${ }^{88}$, A. Z. Wang ${ }^{109}$, C. Wang ${ }^{116}$, C. Wang ${ }^{196}$, H. Wang ${ }^{32}$, H. Wang ${ }^{143}$, J. Wang ${ }^{175}$, P. Wang ${ }^{137}$, Q. Wang ${ }^{2}$, R.-J. Wang ${ }^{15}$, R. Wang ${ }^{116}$, R. Wang ${ }^{12}$, S. M. Wang ${ }^{45}$, W. T. Wang ${ }^{116}$, W. Wang ${ }^{172}$, W. X. Wang ${ }^{116}$, Y. Wang ${ }^{116}$, Z. Wang ${ }^{57}$, C. Wanotayaroj ${ }^{58}$, A. Warburton ${ }^{31}$, C. P. Ward ${ }^{121}$, R. J. Ward ${ }^{49}$, N. Warrack ${ }^{119}$, A. T. Watson ${ }^{49}$, M. F. Watson ${ }^{49}$, G. Watts ${ }^{53}$, B. M. Waugh ${ }^{113}$, A. F. Webb ${ }^{62}$, C. Weber ${ }^{91}$, M. S. Weber ${ }^{63}$, S. A. Weber ${ }^{131}$, S. M. Weber ${ }^{67}$, Y. Wei ${ }^{56}$, A. R. Weidberg ${ }^{56}$, J. Weingarten ${ }^{188}$, M. Weirich ${ }^{15}$, C. Weiser ${ }^{81}$, P. S. Wells ${ }^{4}$, T. Wenaus ${ }^{91}$, B. Wendland ${ }^{188}$, T. Wengler ${ }^{4}$, S. Wenig ${ }^{4}$, N. Wermes ${ }^{123}$, M. Wessels ${ }^{67}$, T. D. Weston ${ }^{63}$, K. Whalen ${ }^{48}$, A. M. Wharton ${ }^{118}$, A. S. White ${ }^{57}$, A. White ${ }^{105}$, M. J. White ${ }^{197}$, D. Whiteson ${ }^{82}$, B. W. Whitmore ${ }^{118}$, W. Wiedenmann ${ }^{109}$, C. Wiel ${ }^{136}$, 
M. Wielers ${ }^{21}$, N. Wieseotte ${ }^{15}$, C. Wiglesworth ${ }^{8}$, L. A. M. Wiik-Fuchs ${ }^{81}$, H. G. Wilkens ${ }^{4}$, L. J. Wilkins ${ }^{6}$, D. M. Williams ${ }^{69}$, H. H. Williams ${ }^{173}$, S. Williams ${ }^{121}$, S. Willocq ${ }^{3}$, P. J. Windischhofer ${ }^{56}$, I. Wingerter-Seez ${ }^{16}$, E. Winkels ${ }^{9}$, F. Winklmeier ${ }^{48}$, B. T. Winter ${ }^{81}$, M. Wittgen ${ }^{115}$, M. Wobisch ${ }^{241}$, A. Wolf ${ }^{15}$, R. Wölker ${ }^{56}$, J. Wollrath ${ }^{81}$, M. W. Wolter ${ }^{101}$, H. Wolters ${ }^{27,202}$, V. W. S. Wong ${ }^{185}$, A. F. Wongel ${ }^{58}$, N. L. Woods ${ }^{22}$, S. D. Worm ${ }^{58}$, B. K. Wosiek ${ }^{101}$, K. W. Woźniak ${ }^{101}$, K. Wraightt19, S. L. Wu ${ }^{109}$, X. Wu ${ }^{20}$, Y. Wu ${ }^{116}$, J. Wuerzinger ${ }^{56}$, T. R. Wyatt ${ }^{142}$, B. M. Wynne ${ }^{167}$, S. Xella ${ }^{8}$, L. Xia ${ }^{127}$, J. Xiang ${ }^{231}$, X. Xiao ${ }^{57}$,

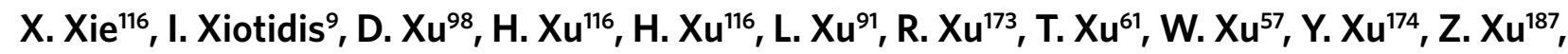
Z. Xu ${ }^{115}$, B. Yabsley ${ }^{106}$, S. Yacoob ${ }^{93}$, D. P. Yallup ${ }^{113}$, N. Yamaguchi ${ }^{26}$, Y. Yamaguchi ${ }^{87}$, A. Yamamoto ${ }^{76}$, M. Yamatani ${ }^{86}$, T. Yamazaki ${ }^{86}$, Y. Yamazaki ${ }^{219}$, J. Yan ${ }^{196}$, Z. Yan ${ }^{157}$, H. J. Yang ${ }^{152,196}$, H. T. Yang ${ }^{32}$, S. Yang ${ }^{116}$, T. Yang ${ }^{231}$, X. Yang ${ }^{116}$, X. Yang ${ }^{183,187}$, Y. Yang ${ }^{86}$, Z. Yang ${ }^{116}$, W-M. Yao ${ }^{32}$, Y. C. Yap ${ }^{58}$, H. Ye ${ }^{172}$, J. Ye ${ }^{137}$, S. Ye ${ }^{91}$, I. Yeletskikh ${ }^{30}$, M. R. Yexley ${ }^{118}$, E. Yigitbasi ${ }^{157}$, P. Yin ${ }^{69}$, K. Yorita ${ }^{225}$, K. Yoshihara ${ }^{59}$, C. J. S. Young ${ }^{4}$, C. Young ${ }^{115}$, J. Yu ${ }^{59}$, R. Yuan ${ }^{187,282}$, X. Yue ${ }^{67}$, M. Zaazoua ${ }^{120}$, B. Zabinski ${ }^{101}$, G. Zacharis ${ }^{42}$, E. Zaffaroni ${ }^{20}$, J. Zahreddine ${ }^{125}$, A. M. Zaitsev ${ }^{148,252}$, T. Zakareishvili ${ }^{171}$, N. Zakharchuk ${ }^{131}$, S. Zambito ${ }^{4}$, D. Zanzi ${ }^{4}$, S. V. Zeißner ${ }^{188}$, C. Zeitnitz ${ }^{97}$, G. Zemaityte ${ }^{56}$, J. C. Zeng ${ }^{95}$, O. Zenin ${ }^{148}$, T. Ženiš ${ }^{92}$, D. Zerwas ${ }^{23}$, M. Zgubič ${ }^{56}$, B. Zhang ${ }^{172}$, D. F. Zhang ${ }^{174}$, G. Zhang ${ }^{174}$, J. Zhang ${ }^{12}$, K. Zhang ${ }^{98}$, L. Zhang ${ }^{172}$, L. Zhang ${ }^{116}$, M. Zhang ${ }^{95}$, R. Zhang ${ }^{109}$, S. Zhang ${ }^{57}$, X. Zhang ${ }^{196}$, X. Zhang ${ }^{187}$, Y. Zhang ${ }^{98,179}$, Z. Zhang ${ }^{175}$, Z. Zhang ${ }^{23}$, P. Zhao ${ }^{79}$, Y. Zhao ${ }^{22}$, Z. Zhao ${ }^{116}$, A. Zhemchugov ${ }^{30}$, Z. Zheng ${ }^{57}$, D. Zhong ${ }^{95}$, B. Zhou ${ }^{57}$, C. Zhou ${ }^{109}$, H. Zhou ${ }^{135}$, M. Zhou ${ }^{130}$, N. Zhou ${ }^{196}$, Y. Zhou ${ }^{135}$, C. G. Zhu' ${ }^{187}$, C. Zhu ${ }^{98,179}$, H. L. Zhu ${ }^{116}$, H. Zhu ${ }^{98}$, J. Zhu ${ }^{57}$, Y. Zhu ${ }^{116}$, X. Zhuang ${ }^{98}$, K. Zhukov ${ }^{37}$, V. Zhulanov ${ }^{70,71}$, D. Zieminska ${ }^{160}$, N. I. Zimine ${ }^{30}$, S. Zimmermann ${ }^{81,283}$, Z. Zinonos ${ }^{114}$, M. Ziolkowski' ${ }^{122}$, L. Živković104, G. Zobernig ${ }^{109}$, A. Zoccoli ${ }^{38,39}$, K. Zoch ${ }^{5}$, T. G. Zorbas ${ }^{60}$, R. Zou ${ }^{205}$ and L. Zwalinski ${ }^{4}$

\footnotetext{
${ }^{1}$ CPPM, Aix-Marseille Université, CNRS/IN2P3, Marseille, France. ${ }^{2}$ Homer L. Dodge Department of Physics and Astronomy, University of Oklahoma, Norman, OK, USA. ${ }^{3}$ Department of Physics, University of Massachusetts, Amherst, MA, USA. ${ }^{4}$ CERN, Geneva, Switzerland. ${ }^{5} I$. Physikalisches Institut, Georg-August-Universität Göttingen, Göttingen, Germany. ${ }^{6}$ Department of Physics, Royal Holloway University of London, Egham, UK. ${ }^{7}$ Department of Physics, University of Toronto, Toronto, Ontario, Canada. ${ }^{8}$ Niels Bohr Institute, University of Copenhagen, Copenhagen, Denmark. ${ }^{9}$ Department of Physics and Astronomy, University of Sussex, Brighton, UK. ${ }^{10}$ Raymond and Beverly Sackler School of Physics and Astronomy, Tel Aviv University, Tel Aviv, Israel. ${ }^{11 D}$ epartment of Physics, Technion, Israel Institute of Technology, Haifa, Israel. ${ }^{2}$ High Energy Physics Division, Argonne National Laboratory, Argonne, IL, USA. ${ }^{13}$ INFN Gruppo Collegato di Udine, Sezione di Trieste, Udine, Italy. ${ }^{14}$ ICTP, Trieste, Italy. ${ }^{15}$ Institut für Physik, Universität Mainz, Mainz, Germany. ${ }^{16}$ LAPP, Université Grenoble Alpes, Université Savoie Mont Blanc, CNRS/IN2P3, Annecy, France. ${ }^{17}$ AGH University of Science and Technology, Faculty of Physics and Applied Computer Science, Krakow, Poland. ${ }^{18}$ Department of Physics, Northern Illinois University, DeKalb, IL, USA. ${ }^{19}$ Department of Physics, Bogazici University, Istanbul, Turkey. ${ }^{20}$ Département de Physique Nucléaire et Corpusculaire, Université de Genéve, Geneva, Switzerland. ${ }^{21}$ Particle Physics Department, Rutherford Appleton Laboratory, Didcot, UK. ${ }^{22}$ Santa Cruz Institute for Particle Physics, University of California Santa Cruz, Santa Cruz, CA, USA. ${ }^{23}$ IJCLab, Université Paris-Saclay, CNRS/IN2P3, Orsay, France. ${ }^{24}$ LPC, Université Clermont Auvergne, CNRS/IN2P3, Clermont-Ferrand, France. ${ }^{25}$ Institute for Mathematics, Astrophysics and Particle Physics, Radboud University/Nikhef, Nijmegen, Netherlands. ${ }^{26}$ Department of Physics, Alexandru loan Cuza University of lasi, lasi, Romania. ${ }^{27}$ Laboratório de Instrumentação e Física Experimental de Partículas - LIP, Lisbon, Portugal. ${ }^{28}$ Departamento de Física Teórica y del Cosmos, Universidad de Granada, Granada, Spain. ${ }^{29}$ Dep Física and CEFITEC of Faculdade de Ciências e Tecnologia, Universidade Nova de Lisboa, Caparica, Portugal. ${ }^{30}$ Joint Institute for Nuclear Research, Dubna, Russia. ${ }^{31}$ Department of Physics, McGill University, Montreal, Quebec, Canada. ${ }^{32}$ Physics Division, Lawrence Berkeley National Laboratory and University of California, Berkeley, CA, USA. ${ }^{33}$ INFN Sezione di Roma Tor Vergata, Rome, Italy. ${ }^{34}$ Dipartimento di Fisica, Università di Roma Tor Vergata, Rome, Italy. ${ }^{35} \mathrm{Faculty}$ of Science, Kyoto University, Kyoto, Japan. ${ }^{36} \mathrm{Fysiska} \mathrm{Institutionen,}$ Lunds Universitet, Lund, Sweden. ${ }^{37}$ P.N. Lebedev Physical Institute of the Russian Academy of Sciences, Moscow, Russia. ${ }^{38}$ Dipartimento di Fisica, INFN Bologna and Università di Bologna, Bologna, Italy. ${ }^{39}$ INFN Sezione di Bologna, Bologna, Italy. ${ }^{40}$ Department of Physics and Astronomy, University of Victoria, Victoria, British Columbia, Canada. ${ }^{41} \mathrm{H}$ oria Hulubei National Institute of Physics and Nuclear Engineering, Bucharest, Romania. ${ }^{42}$ Physics Department, National Technical University of Athens, Zografou, Greece. ${ }^{43}$ Nikhef National Institute for Subatomic Physics and University of Amsterdam, Amsterdam, Netherlands. ${ }^{44}$ Czech Technical University in Prague, Prague, Czech Republic. ${ }^{45}$ Institute of Physics, Academia Sinica, Taipei, Taiwan. ${ }^{46}$ Tomsk State University, Tomsk, Russia. ${ }^{47}$ INFN Sezione di Milano, Milan, Italy. ${ }^{48}$ Institute for Fundamental Science, University of Oregon, Eugene, OR, USA. ${ }^{49}$ School of Physics and Astronomy, University of Birmingham, Birmingham, UK. ${ }^{50}$ INFN Sezione di Napoli, Naples, Italy. ${ }^{51}$ Dipartimento di Fisica, Università di Napoli, Naples, Italy. ${ }^{52}$ Instituto de Física La Plata, Universidad Nacional de La Plata and CONICET, La Plata, Argentina. ${ }^{53}$ Department of Physics, University of Washington, Seattle, WA, USA. ${ }^{54}$ Departamento de Física Teorica C-15 and CIAFF, Universidad Autónoma de Madrid, Madrid, Spain. ${ }^{55}$ Universidade Federal do Rio De Janeiro COPPE/EE/IF, Rio de Janeiro, Brazil. ${ }^{56}$ Department of Physics, Oxford University, Oxford, UK. ${ }^{57}$ Department of Physics, University of Michigan, Ann Arbor, MI, USA. ${ }^{58}$ Deutsches Elektronen-Synchrotron DESY, Hamburg and Zeuthen, Germany. ${ }^{59}$ Department of Physics and Astronomy, lowa State University, Ames, IA, USA. ${ }^{0}$ Department of Physics and Astronomy, University of Sheffield, Sheffield, UK. ${ }^{61}$ IRFU, CEA, Université Paris-Saclay, Gif-sur-Yvette, France. ${ }^{62}$ Department of Physics, University of Texas at Austin, Austin, TX, USA. ${ }^{63}$ Albert Einstein Center for Fundamental Physics and Laboratory for High Energy Physics, University of Bern, Bern, Switzerland. ${ }^{64}$ Department of Physics, Stockholm University, Stockholm, Sweden. ${ }^{65}$ Oskar Klein Centre, Stockholm, Sweden. ${ }^{66}$ Dipartimento di Fisica, Università di Milano, Milan, Italy. ${ }^{67}$ Kirchhoff-Institut für Physik, Ruprecht-Karls-Universität Heidelberg, Heidelberg, Germany. ${ }^{68}$ Physics Department, National and Kapodistrian University of Athens, Athens, Greece.
} 
${ }^{69}$ Nevis Laboratory, Columbia University, Irvington, NY, USA. ${ }^{70}$ Budker Institute of Nuclear Physics and NSU, SB RAS, Novosibirsk, Russia. ${ }^{71}$ Novosibirsk State University Novosibirsk, Novosibirsk, Russia. ${ }^{72}$ INFN Sezione di Pisa, Pisa, Italy. ${ }^{73}$ Department of Physics, Oklahoma State University, Stillwater, OK, USA. ${ }^{74}$ INFN e Laboratori Nazionali di Frascati, Frascati, Italy. ${ }^{75}$ INFN Sezione di Roma, Rome, Italy. ${ }^{76} \mathrm{KEK}$, High Energy Accelerator Research Organization, Tsukuba, Japan. ${ }^{77}$ Instituto de Física Corpuscular (IFIC), Centro Mixto Universidad de Valencia - CSIC, Valencia, Spain. ${ }^{78}$ Departamento de Engenharia Elétrica, Universidade Federal de Juiz de Fora (UFJF), Juiz de Fora, Brazil. ${ }^{79}$ Department of Physics, Duke University, Durham, NC, USA. ${ }^{80}$ Group of Particle Physics, University of Montreal, Montreal, Quebec, Canada. ${ }^{81}$ Physikalisches Institut, Albert-Ludwigs-Universität Freiburg, Freiburg, Germany. ${ }^{82}$ Department of Physics and Astronomy, University of California Irvine, Irvine, CA, USA. ${ }^{83}$ Fakultät für Physik, Ludwig-Maximilians-Universität München, Munich, Germany. ${ }^{84}$ Graduate School of Science and Kobayashi-Maskawa Institute, Nagoya University, Nagoya, Japan. ${ }^{85}$ Ochanomizu University, Tokyo, Japan. ${ }^{86}$ International Center for Elementary Particle Physics and Department of Physics, University of Tokyo, Tokyo, Japan. ${ }^{87}$ Department of Physics, Tokyo Institute of Technology, Tokyo, Japan. ${ }^{88}$ Laboratory for Particle Physics and Cosmology, Harvard University, Cambridge, MA, USA. ${ }^{89}$ Department of Physics and Astronomy, University of Uppsala, Uppsala, Sweden. ${ }^{90}$ Faculté des Sciences, Université Mohamed Premier and LPTPM, Oujda, Morocco. ${ }^{91}$ Physics Department, Brookhaven National Laboratory, Upton, NY, USA. ${ }^{92}$ Faculty of Mathematics, Physics and Informatics, Comenius University, Bratislava, Slovakia. ${ }^{93}$ Department of Physics, University of Cape Town, Cape Town, South Africa. ${ }^{94}$ iThemba Labs, Western Cape, South Africa. ${ }^{95}$ Department of Physics, University of Illinois, Urbana, IL, USA. ${ }^{96}$ Institut für Physik, Humboldt Universität zu Berlin, Berlin, Germany. ${ }^{97}$ Fakultät für Mathematik und Naturwissenschaften, Fachgruppe Physik, Bergische Universität Wuppertal, Wuppertal, Germany. ${ }^{98}$ Institute of High Energy Physics, Chinese Academy of Sciences, Beijing, China. ${ }^{99}$ Department of Physics, Aristotle University of Thessaloniki, Thessaloniki, Greece. ${ }^{100}$ Dipartimento di Fisica, Sapienza Università di Roma, Rome, Italy. ${ }^{101}$ Institute of Nuclear Physics Polish Academy of Sciences, Krakow, Poland. ${ }^{102}$ Department of Physics, Simon Fraser University, Burnaby, British Columbia, Canada. ${ }^{103}$ Department of Physics, Yale University, New Haven, CT, USA. ${ }^{104}$ Institute of Physics, University of Belgrade, Belgrade, Serbia. ${ }^{105}$ Department of Physics, University of Texas at Arlington, Arlington, TX, USA. ${ }^{106}$ School of Physics, University of Sydney, Sydney, New South Wales, Australia. ${ }^{107}$ Department of Particle Physics and Astrophysics, Weizmann Institute of Science, Rehovot, Israel. ${ }^{108}$ Department of Physics and Astronomy, University of Pittsburgh, Pittsburgh, PA, USA. ${ }^{109}$ Department of Physics, University of Wisconsin, Madison, WI, USA. ${ }^{10}$ School of Physics, University of Melbourne, Melbourne, Victoria, Australia. "'1"Dipartimento di Fisica, Università di Genova, Genova, Italy. ${ }^{112}$ INFN Sezione di Genova, Genova, Italy. ${ }^{113}$ Department of Physics and Astronomy, University College London, London, UK. ${ }^{114}$ Max-Planck-Institut für Physik (Werner-Heisenberg-Institut), Munich, Germany. ${ }^{115}$ SLAC National Accelerator Laboratory, Stanford, CA, USA. ${ }^{116}$ Department of Modern Physics and State Key Laboratory of Particle Detection and Electronics, University of Science and Technology of China, Hefei, China. ${ }^{17}$ Konstantinov Nuclear Physics Institute of National Research Centre 'Kurchatov Institute', PNPI, St Petersburg, Russia. ${ }^{118}$ Physics Department, Lancaster University, Lancaster, UK. ${ }^{119}$ SUPA - School of Physics and Astronomy, University of Glasgow, Glasgow, UK. ${ }^{120}$ Faculté des Sciences, Université Mohammed V, Rabat, Morocco. ${ }^{121}$ Cavendish Laboratory, University of Cambridge, Cambridge, UK. ${ }^{122}$ Department Physik, Universität Siegen, Siegen, Germany. ${ }^{123}$ Physikalisches Institut, Universität Bonn, Bonn, Germany. ${ }^{124}$ California State University, Long Beach, CA, USA. ${ }^{125}$ LPNHE, Sorbonne Université, Université de Paris, CNRS/IN2P3, Paris, France. ${ }^{126}$ Department of Physics and Astronomy, Tufts University, Medford, MA, USA. ${ }^{127}$ Department of Physics, University of Warwick, Coventry, UK. ${ }^{128}$ Department of Physics Engineering, Gaziantep University, Gaziantep, Turkey. ${ }^{129}$ Bahcesehir University, Faculty of Engineering and Natural Sciences, Istanbul, Turkey. ${ }^{130}$ Departments of Physics and Astronomy, Stony Brook University, Stony Brook, NY, USA. ${ }^{131}$ Department of Physics, Carleton University, Ottawa, Ontario, Canada. ${ }^{132}$ National Research Nuclear University MEPhl, Moscow, Russia. ${ }^{133}$ Faculté des Sciences Ain Chock, Réseau Universitaire de Physique des Hautes Energies - Université Hassan II, Casablanca, Morocco. ${ }^{134}$ Department of Physics, Brandeis University, Waltham, MA, USA. ${ }^{135}$ Department of Physics, University of Arizona, Tucson, AZ, USA. ${ }^{136}$ Institut für Kern- und Teilchenphysik, Technische Universität Dresden, Dresden, Germany. ${ }^{137}$ Physics Department, Southern Methodist University, Dallas, TX, USA. ${ }^{138}$ School of Physics and Astronomy, Queen Mary University of London, London, UK. ${ }^{139}$ Fakultät für Physik und Astronomie, Julius-Maximilians-Universität Würzburg, Würzburg, Germany. ${ }^{140}$ Dipartimento di Fisica E. Fermi, Università di Pisa, Pisa, Italy. ${ }^{141}$ INFN Sezione di Roma Tre, Rome, Italy. ${ }^{142}$ School of Physics and Astronomy, University of Manchester, Manchester, UK. ${ }^{143}$ Department of Physics, University of Alberta, Edmonton, Alberta, Canada. ${ }^{144}$ Department of Physics, University of Oslo, Oslo, Norway. ${ }^{145}$ Institut de Física d'Altes Energies (IFAE), Barcelona Institute of Science and Technology, Barcelona, Spain. ${ }^{146}$ Physikalisches Institut, Ruprecht-Karls-Universität Heidelberg, Heidelberg, Germany. ${ }^{147}$ Oliver Lodge Laboratory, University of Liverpool, Liverpool, UK. ${ }^{148}$ Institute for High Energy Physics of the National Research Centre Kurchatov Institute, Protvino, Russia. ${ }^{149}$ Ohio State University, Columbus, OH, USA. ${ }^{150}$ Department of Mechanical Engineering Science, University of Johannesburg, Johannesburg, South Africa. ${ }^{151}$ Department of Physics, University of South Africa, Pretoria, South Africa. ${ }^{152}$ Tsung-Dao Lee Institute, Shanghai, China. ${ }^{153}$ Department of Physics and Astronomy, Michigan State University, East Lansing, MI, USA. ${ }^{154}$ Departamento de Física, Universidad Técnica Federico Santa María, Valparaíso, Chile. ${ }^{155}$ Department of Subnuclear Physics, Institute of Experimental Physics of the Slovak Academy of Sciences, Kosice, Slovak Republic. ${ }^{156}$ Department for Physics and Technology, University of Bergen, Bergen, Norway. ${ }^{157}$ Department of Physics, Boston University, Boston, MA, USA. ${ }^{158}$ Physikalisches Institut II, Justus-Liebig-Universität Giessen, Giessen, Germany. ${ }^{159}$ Department of Physics, Ankara University, Ankara, Turkey. ${ }^{160}$ Department of Physics, Indiana University, Bloomington, IN, USA. ${ }^{161}$ Dipartimento di Matematica e Fisica, Università Roma Tre, Rome, Italy. ${ }^{162}$ University of lowa, lowa City, IA, USA. ${ }^{163}$ Dipartimento di Fisica, Università della Calabria, Rende, Italy. ${ }^{164}$ INFN Gruppo Collegato di Cosenza, Laboratori Nazionali di Frascati, Frascati, Italy. ${ }^{165}$ Charles University, Faculty of Mathematics and Physics, Prague, Czech Republic. ${ }^{166}$ TRIUMF, Vancouver, British Columbia, Canada. ${ }^{167}$ SUPA - School of Physics and Astronomy, University of Edinburgh, Edinburgh, UK. ${ }^{168}$ Departamento de Física, Universidade do Minho, Braga, Portugal. ${ }^{169}$ Istanbul Bilgi University, Faculty of Engineering and Natural Sciences, Istanbul, Turkey. ${ }^{170}$ Palacký University, RCPTM, Joint Laboratory of Optics, Olomouc, Czech Republic. ${ }^{171} \mathrm{High}$ Energy Physics Institute, Tbilisi State University, Tbilisi, Georgia. ${ }^{172}$ Department of Physics, Nanjing University, Nanjing, China. ${ }^{173}$ Department of Physics, University of Pennsylvania, Philadelphia, PA, USA. ${ }^{174}$ Physics Department, Tsinghua University, Beijing, China.

${ }^{175}$ Department of Physics, Chinese University of Hong Kong, Hong Kong, China. ${ }^{176}$ Department of Physics, National Tsing Hua University, Hsinchu, Taiwan. ${ }^{177}$ INFN Sezione di Lecce, Zona Monte, Italy. ${ }^{178}$ School of Physics, University of the Witwatersrand, Johannesburg, South Africa. ${ }^{179}$ University of Chinese Academy of Science (UCAS), Beijing, China. ${ }^{180}$ Institute of Physics of the Czech Academy of Sciences, Prague, Czech Republic. ${ }^{181}$ Department of Experimental Particle Physics, Jožef Stefan Institute and Department of Physics, University of Ljubljana, Ljubljana, Slovenia. ${ }^{182}$ Dipartimento Politecnico di Ingegneria e Architettura, Università di Udine, Udine, Italy. ${ }^{183}$ LPSC, Université Grenoble Alpes, CNRS/IN2P3, Grenoble INP, Grenoble, France. ${ }^{184}$ Instituto Superior Técnico, Universidade de Lisboa, Lisbon, Portugal. ${ }^{185}$ Department of Physics, University of British Columbia, Vancouver, British Columbia, Canada. ${ }^{186}$ Department of Physics, New York University, New York, NY, USA. ${ }^{187}$ Institute of Frontier and Interdisciplinary Science and Key Laboratory of Particle Physics and Particle Irradiation (MOE), Shandong University, Qingdao, China. ${ }^{188}$ Lehrstuhl für Experimentelle Physik IV, Technische Universität Dortmund, Dortmund, Germany. ${ }^{189}$ Departamento de Física, Universidad de Buenos Aires, Buenos Aires, Argentina. ${ }^{190}$ Department of Physics and Astronomy, York University, Toronto, Ontario, Canada. ${ }^{191}$ INFN-TIFPA, Trento, Italy. ${ }^{192}$ Università degli Studi di Trento, Trento, Italy. ${ }^{193}$ Departamento de Física, Pontificia Universidad Católica de Chile, Santiago, Chile. ${ }^{194}$ Universidade Federal de São João del Rei (UFSJ), São João del Rei, Brazil. ${ }^{195}$ Instituto de Física, Universidade de São Paulo, São Paulo, Brazil. ${ }^{196}$ School of Physics and Astronomy, Shanghai Jiao Tong University, KLPPAC-MoE, SKLPPC, Shanghai, China. ${ }^{197}$ Department of Physics, University of Adelaide, Adelaide, South Australia, Australia. ${ }^{198}$ National Centre for Scientific Research 'Demokritos', Agia Paraskevi, Greece. ${ }^{199}$ INFN Sezione di Pavia, Pavia, Italy. ${ }^{200}$ Dipartimento di Fisica, Università di Pavia, Pavia, Italy. ${ }^{201}$ Physics Department, University of Texas at Dallas, Richardson, TX, USA. ${ }^{202}$ Departamento de Física, Universidade de Coimbra, Coimbra, Portugal. ${ }^{203}$ Graduate School of Science and Technology, 
Tokyo Metropolitan University, Tokyo, Japan. ${ }^{204}$ Nagasaki Institute of Applied Science, Nagasaki, Japan. ${ }^{205}$ Enrico Fermi Institute, University of Chicago, Chicago, IL, USA. ${ }^{206}$ Institute for Theoretical and Experimental Physics named by A.I. Alikhanov of National Research Centre 'Kurchatov Institute', Moscow, Russia. ${ }^{207}$ Marian Smoluchowski Institute of Physics, Jagiellonian University, Krakow, Poland. ${ }^{208}$ D.V. Skobeltsyn Institute of Nuclear Physics, M.V. Lomonosov Moscow State University, Moscow, Russia. ${ }^{209}$ Departamento de Física, Faculdade de Ciências, Universidade de Lisboa, Lisbon, Portugal.

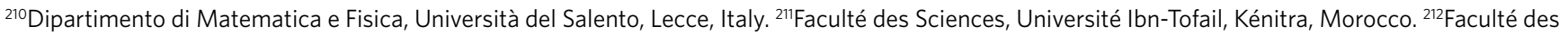
Sciences Semlalia, Université Cadi Ayyad, LPHEA-Marrakech, Marrakesh, Morocco. ${ }^{213}$ West University in Timisoara, Timisoara, Romania. ${ }^{214}$ Department of Physics and Astronomy, University of New Mexico, Albuquerque, NM, USA. ${ }^{215}$ Institut für Astro- und Teilchenphysik, Leopold-Franzens-Universität, Innsbruck, Austria. ${ }^{216}$ Division of Physics and Tomonaga Center for the History of the Universe, Faculty of Pure and Applied Sciences, University of Tsukuba, Tsukuba, Japan. ${ }^{217}$ B.I. Stepanov Institute of Physics, National Academy of Sciences of Belarus, Minsk, Belarus. ${ }^{218}$ Department of Physics, Shinshu University, Nagano, Japan. ${ }^{219}$ Graduate School of Science, Kobe University, Kobe, Japan. ${ }^{220}$ Graduate School of Science, Osaka University, Osaka, Japan. ${ }^{221}$ University Politehnica Bucharest, Bucharest, Romania. ${ }^{222}$ Research Institute for Nuclear Problems of Byelorussian State University, Minsk, Belarus. ${ }^{223}$ Physics Department, SUNY Albany, Albany, NY, USA. ${ }^{224}$ E. Andronikashvili Institute of Physics, Iv. Javakhishvili Tbilisi State University, Tbilisi, Georgia. ${ }^{225}$ Waseda University, Tokyo, Japan. ${ }^{226}$ Research Center for Advanced Particle Physics and Department of Physics, Kyushu University, Fukuoka, Japan. ${ }^{227}$ Institute of Physics, Azerbaijan Academy of Sciences, Baku, Azerbaijan. ${ }^{228}$ Istanbul Aydin University, Application and Research Center for Advanced Studies, Istanbul, Turkey. ${ }^{229}$ Universidad Andres Bello, Department of Physics, Santiago, Chile. ${ }^{230}$ Instituto de Alta Investigación, Universidad de Tarapacá, Arica, Chile. ${ }^{231}$ Department of Physics and Institute for Advanced Study, Hong Kong University of Science and Technology, Hong Kong, China. ${ }^{232}$ Department of Physics, University of Hong Kong, Hong Kong, China. ${ }^{233}$ Physics Department, Royal Institute of Technology, Stockholm, Sweden. ${ }^{234}$ Centro de Física Nuclear da Universidade de Lisboa, Lisbon, Portugal. ${ }^{235}$ Facultad de Ciencias y Centro de Investigaciónes, Universidad Antonio Nariño, Bogota, Colombia. ${ }^{236} \mathrm{Faculty}$ of Applied Information Science, Hiroshima Institute of Technology, Hiroshima, Japan. ${ }^{237}$ Transilvania University of Brasov, Brasov, Romania. ${ }^{238}$ Physics Department, National Institute for Research and Development of Isotopic and Molecular Technologies, Cluj-Napoca, Romania. ${ }^{239}$ Centre de Calcul de I'Institut National de Physique Nucléaire et de Physique des Particules (IN2P3), Villeurbanne, France. ${ }^{240}$ Departamento de Física, Universidad Nacional de Colombia, Bogota, Colombia. ${ }^{24}$ Louisiana Tech University, Ruston, LA, USA. ${ }^{242}$ Division of Physics, TOBB University of Economics and Technology, Ankara, Turkey. ${ }^{243}$ Kyoto University of Education, Kyoto, Japan. ${ }^{244}$ Present address: Department of Physics, King's College London, London, UK. ${ }^{245}$ Present address: Department of Physics, Istanbul University, Istanbul, Turkey. ${ }^{246}$ Present address: Instituto de Fisica Teorica, IFT-UAM/CSIC, Madrid, Spain. ${ }^{247}$ Present address: TRIUMF, Vancouver, British Columbia, Canada. ${ }^{248}$ Present address: Department of Physics and Astronomy, University of Louisville, Louisville, KY, USA. ${ }^{249}$ Present address: Physics Department, An-Najah National University, Nablus, Palestine. ${ }^{250}$ Present address: Department of Physics, University of Fribourg, Fribourg, Switzerland. 251Present address: Departament de Fisica de la Universitat Autonoma de Barcelona, Barcelona, Spain. ${ }^{252}$ Present address: Moscow Institute of Physics and Technology State University, Dolgoprudny, Russia. ${ }^{253}$ Present address: Department of Physics, Ben Gurion University of the Negev, Beer Sheva, Israel. ${ }^{254}$ Present address: Università di Napoli Parthenope, Naples, Italy. ${ }^{255}$ Present address: Institute of Particle Physics (IPP), Victoria, British Columbia, Canada. ${ }^{256}$ Present address: Department of Physics, St Petersburg State Polytechnical University, St Petersburg, Russia. ${ }^{257}$ Present address: Borough of Manhattan Community College, City University of New York, New York, NY, USA. ${ }^{258}$ Present address: Department of Physics, California State University, Fresno, CA, USA. ${ }^{259}$ Present address: Department of Financial and Management Engineering, University of the Aegean, Chios, Greece. ${ }^{260}$ Present address: Centro Studi e Ricerche Enrico Fermi, Rome, Italy. ${ }^{261}$ Present address: Department of Physics, California State University, East Bay, CA, USA. ${ }^{262}$ Present address: Institucio Catalana de Recerca i Estudis Avancats, ICREA, Barcelona, Spain. ${ }^{263}$ Present address: Graduate School of Science, Osaka University, Osaka, Japan. ${ }^{264}$ Present address: Physikalisches Institut, Albert-Ludwigs-Universität Freiburg, Freiburg, Germany. ${ }^{265}$ Present address: University of Chinese Academy of Sciences (UCAS), Beijing, China. ${ }^{266}$ Present address: Institute of Physics, Azerbaijan Academy of Sciences, Baku, Azerbaijan. ${ }^{267}$ Present address: CERN, Geneva, Switzerland. ${ }^{268}$ Present address: Joint Institute for Nuclear Research, Dubna, Russia. ${ }^{269}$ Present address: Hellenic Open University, Patras, Greece. ${ }^{270}$ Present address: Center for High Energy Physics, Peking University, Beijing, China. ${ }^{271}$ Present address: The City College of New York, New York, NY, USA. ${ }^{272}$ Present address: Dipartimento di Matematica, Informatica e Fisica, Università di Udine, Udine, Italy. ${ }^{273}$ Present address: Department of Physics, California State University, Sacramento, CA, USA. ${ }^{274}$ Present address: Département de Physique Nucléaire et Corpusculaire, Université de Genéve, Geneva, Switzerland. ${ }^{275}$ Present address: Institute for Nuclear Research and Nuclear Energy (INRNE) of the Bulgarian Academy of Sciences, Sofia, Bulgaria. ${ }^{276}$ Present address: Faculty of Physics, M.V. Lomonosov Moscow State University, Moscow, Russia. ${ }^{277}$ Present address: Institut für Experimentalphysik, Universität Hamburg, Hamburg, Germany. ${ }^{278}$ Present address: CPPM, Aix-Marseille Université, Marseille, France. ${ }^{279}$ Present address: National Research Nuclear University MEPhl, Moscow, Russia. ${ }^{280}$ Present address: Institute for Particle and Nuclear Physics, Wigner Research Centre for Physics, Budapest, Hungary. ${ }^{281}$ Present address: Giresun University, Faculty of Engineering, Giresun, Turkey. ${ }^{282}$ Present address: Department of Physics and Astronomy, Michigan State University, East Lansing, MI, USA. ${ }^{283}$ Deceased: A. Ouraou, S. Zimmermann. 凶e-mail: atlas.publications@cern.ch 


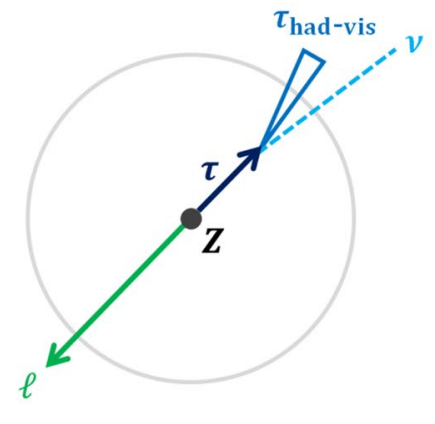

(a)

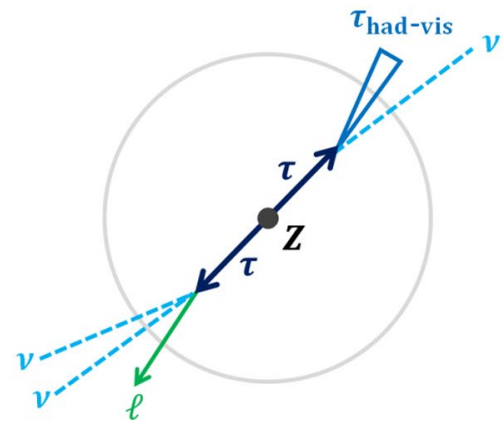

(b)

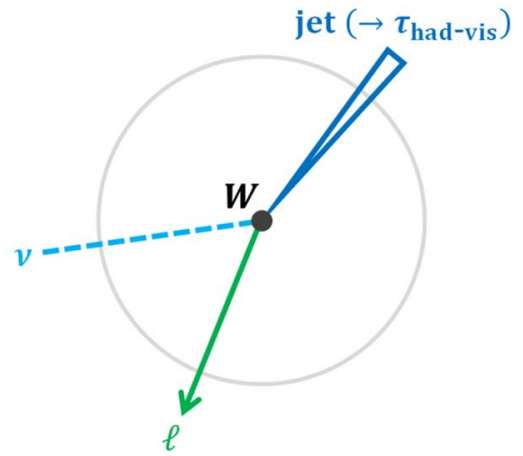

(c)

Extended Data Fig. 1 | Schematic representation of a typical event selected in the SR. The topology as seen in the plane transverse to the beam line is shown. (a) A signal $Z \rightarrow \ell \tau$ event. (b) A $Z \rightarrow \tau \tau$ event. (c) A $W+$ jets event. The green arrows represent reconstructed light leptons $(\ell$ ). The blue triangles represent the $\tau_{\text {had-vis }}$ candidates. The light blue dashed lines represent neutrinos that escape detection and are reconstructed as (part of) the missing transverse momentum of the event. 


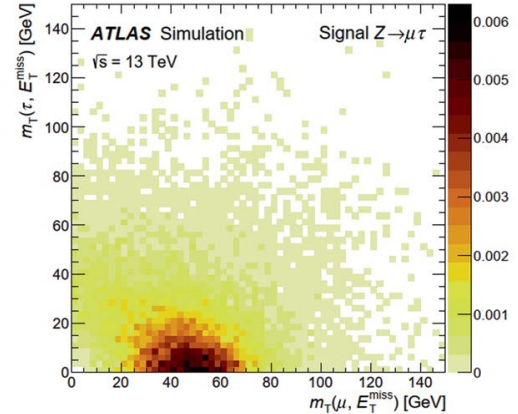

(a)

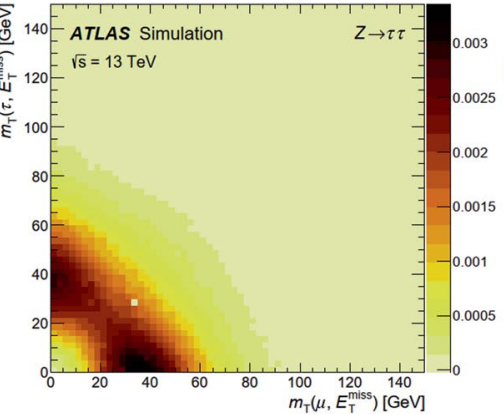

(b)

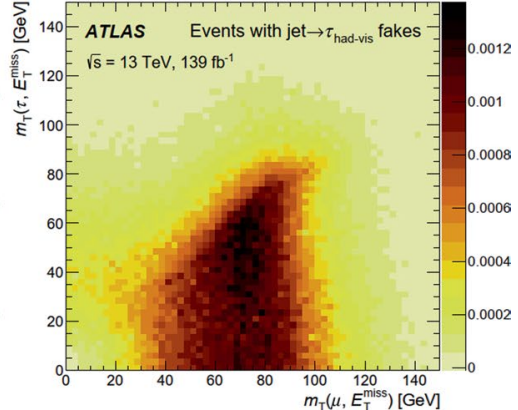

(c)

Extended Data Fig. 2 | Distributions of $\boldsymbol{m}_{\boldsymbol{T}}\left(\tau_{\text {had-vis, }} E_{\mathrm{T}}^{\text {miss }}\right)$ versus $\boldsymbol{m}_{\boldsymbol{T}}\left(\mu, E_{\mathrm{T}}^{\mathrm{miss}}\right)$ of events selected in the SR. (a) Simulated $Z \rightarrow \mu \tau$ events. (b) Simulated $Z \rightarrow \tau \tau$ events. (c) Events measured in data in regions where quark- or gluon-initiated jets are misidentified as $\tau_{\text {had-vis }}$ candidates (events with jet $\rightarrow \tau_{\text {had-vis }}$ fakes, see 'Signal and background predictions' section) in the $\mu \tau$ final state. The colour map represents the fraction of events in each bin. 